\title{
A Nonlinear Multiscale Theory of Atmospheric Blocking: Dynamical and Thermodynamic Effects of Meridional Potential Vorticity Gradient
}

\author{
DEHAi LUO AND WENQI ZHANG \\ Key Laboratory of Regional Climate-Environment for Temperate East Asia, Institute of Atmospheric Physics, Chinese \\ Academy of Science, and University of Chinese Academy of Sciences, Beijing, China
}

(Manuscript received 2 January 2020, in final form 1 April 2020)

\begin{abstract}
In this paper, an extended nonlinear multiscale interaction model is proposed to examine nonlinear behavior of eddy-driven blocking as a Rossby wave packet in a three-dimensional background flow by dividing the background meridional potential vorticity gradient $\left(\mathrm{PV}_{y}\right)$ into dynamical $\mathrm{PV}_{y}\left(\mathrm{PV}_{y}^{D}\right)$ related to the horizontal (mainly meridional) shear of background westerly wind (BWW) and thermodynamic $\mathrm{PV}_{y}\left(\mathrm{PV}_{y}^{T}\right)$ associated with the meridional temperature gradient (MTG). It is found that eddy-driven baroclinic blocking with large amplitude in the midtroposphere tends to have a longer lifetime ( $\sim 20$ days) in a baroclinic atmosphere with stratification than eddy-driven barotropic blocking without vertical variation (less than 15 days). It is shown that barotropic blocking shows a northwest-southeast orientation and has long lifetime, large retrogression, and slow decay only for weaker barotropic $\mathrm{BWW}$ and $\mathrm{PV}_{y}^{D}$ in higher latitudes. In a baroclinic atmosphere with stratification, baroclinic blocking shows long lifetime, strong eastward movement, slow decay, weak strength, and less local persistence for large barotropic BWW and $\mathrm{PV}_{y}^{D}$ under $\mathrm{PV}_{y}^{T}=0$, but becomes less slow decay, weak retrogression, and large local persistence for small barotropic BWW and $\mathrm{PV}_{y}^{D}$. Such a blocking with a north-south antisymmetric dipole, large amplitude, and long local persistence, characterized by a persistent large meander of westerly jet streams, is easily seen when baroclinic BWW and $\mathrm{PV}_{y}^{T}$ are small in the lower to midtroposphere. Comparatively, the magnitude of $\mathrm{PV}_{y}^{T}$ plays a larger role in the blocking change than that of $\mathrm{PV}_{y}^{D}$, whereas the vertical variation of MTG is more important for the blocking change than the MTG itself for some cases.
\end{abstract}

\section{Introduction}

Atmospheric blocking is a large-scale, quasi-stationary, low-frequency circulation pattern with the lifetime of 10-20 days occurring in midlatitudes (Berggren et al. 1949; Rex 1950; Shukla and Mo 1983). Because the extreme cold spells in winter (Buehler et al. 2011), European temperature extremes in spring (Brunner et al. 2017) and heat waves in summer (Della-Marta et al. 2007; Schaller et al. 2018) are often related to the establishment and maintenance of mid-high-latitude blocking, the formation and maintenance mechanism of atmospheric blocking has been an important research topic in past decades (Yeh 1949; Shutts 1983; Colucci 1985; Haines and Marshall 1987; Holopainen and Fortelius 1987; Luo 2000, 2005; Luo et al. 2014, 2019; Zhang and Luo 2020; Nakamura and Huang 2017, 2018; Aikawa et al. 2019; Paradise et al. 2019).

\footnotetext{
Corresponding author: Dr. Dehai Luo, ldh@mail.iap.ac.cn
}

The numerical experiments have indicated that highfrequency synoptic-scale eddies play a more important role in the generation and maintenance of atmospheric blocking than large-scale topographic forcing ( $\mathrm{Ji}$ and Tibaldi 1983). Some diagnostic studies have also revealed that the time-mean eddy vorticity flux induced by high-frequency eddies tends to maintain a time-mean blocking anomaly (Shutts 1983; Illari and Marshall 1983; Holopainen and Fortelius 1987; Mullen 1987; Nakamura and Wallace 1993; Aikawa et al. 2019). However, in the previous studies it is difficult to infer the causal relationship between the variation of synoptic-scale eddies and the evolution of blocking from a time-mean perspective, when a time-mean potential vorticity (PV) equation is used (Holopainen and Fortelius 1987). In fact, in the unfiltered daily geopotential height fields the intensified blocking flow behaves as an enhanced meandering of westerly jet streams (Berggren et al. 1949) due to the intensified multiple vortex structure within the blocking region resulting from the northward 
(southward) displacement of intensified synoptic-scale ridges (deepened troughs) in the upstream side of blocking (Luo 2000, 2005). Such a poleward (equatorward) movement of small-scale ridges (troughs) reflects the presence of cyclonic wave breaking (hereafter CWB) or eddy straining. Thus, in some previous studies the eddy straining or CWB was understood as leading to the blocking formation and maintenance (Shutts 1983; Weijenborg et al. 2012).

Shutts (1983) first attributed the maintenance of dipole blocking to the meridional straining of synopticscale eddies in a diffluent basic flow or a stationary blocking. However, from the temporal evolution of the planetary-scale blocking field and synoptic-scale eddies during their interaction in a nonlinear theoretical model of blocking Luo $(2000,2005)$ demonstrated that the preexisting incident synoptic-scale eddies rather than the eddy straining or CWB are a key driver of the spatiotemporal evolution of blocking and its life cycle (formation, maintenance, and decay). This nonlinear model can describe the life cycle of a blocking event or a strong westerly jet meandering, which is different from the "traffic jam" model of blocking advanced by Nakamura and his collaborators (Nakamura and Huang 2017, 2018; Paradise et al. 2019), because the traffic jam model can only reflect the initial development of blocking as an accumulation of local wave activity rather than its life process. While a dipole blocking can be established by preexisting synoptic-scale eddies (Luo 2000, 2005), the feedback of the intensified blocking has been shown to cause the deformation (straining and merging) of preexisting synoptic-scale eddies or CWB in a nonlinear multiscale interaction (NMI) model (Luo et al. 2014). Thus, Luo et al. (2019) further concluded that the eddy straining or CWB is a result or concomitant phenomenon of the blocking establishment and maintenance rather than a cause. They also found that a small meridional $\mathrm{PV}$ gradient $\left(\mathrm{PV}_{y}\right)$ as a favorable background media tends to maintain blocking through reducing its energy dispersion and strengthening its nonlinearity especially in high latitudes.

However, the previous NMI model is not appropriate for investigating the effect of Arctic amplification (AA) on atmospheric blocking because AA can reduce the meridional temperature gradient (Newson 1973; Cohen et al. 2020) in addition to the change of background westerly wind in mid-high latitudes as well as because this barotropic model ignores the effect of reduced meridional temperature gradient (Luo et al. 2018, 2019). Thus, it is useful to extend our previous barotropic NMI model to include the effect of a three-dimensional background flow. Using this extended NMI model, it becomes feasible to distinguish the different roles of the horizontal (mainly meridional) shear of background westerly wind (BWW) and the magnitude of meridional temperature gradient in the blocking change. This extended NMI model provides a useful theoretical tool for further understanding how AA, continental snow cover, and internal variability influence the evolution of blocking and the generation cause of winter cold extremes.

This paper is organized as follows: In section 2, we describe the blocking-eddy interaction equations in a three-dimensional baroclinic framework as an extended NMI model. In this section, a further simplification of the blocking-eddy interaction equations is also made under some assumptions. The analytical solution of the extended NMI model is presented in section 3. In section 4, we describe theoretical results about how the meridional shear of the BWW and its vertical shear related to the meridional temperature gradient influence the spatiotemporal evolution of eddy-driven blocking wave packet in a barotropic atmosphere without vertical variation and a baroclinic atmosphere with stratification. The main conclusion and discussions are summarized in the final section.

\section{Model description}

We consider a blocking flow governed by a quasigeostrophic baroclinic potential vorticity (PV) equation on a $\beta$ plane in the form

$$
\frac{\partial q_{T}}{\partial t}+J\left(\psi_{T}, q_{T}\right)=\Gamma_{T}-D_{T},
$$

where $\quad q_{T}(x, y, z, t)=f_{0}+\beta_{0} y+\nabla^{2} \psi_{T}+\left(1 / \rho_{0}\right)(\partial / \partial z)$ $\left[\rho_{0}\left(f_{0}^{2} / N^{2}\right)\left(\partial \psi_{T} / \partial z\right)\right]$ is the quasigeostrophic baroclinic PV with $\psi_{T}(x, y, z, t)$ being the total streamfunction; $\nabla^{2}=\partial^{2} / \partial x^{2}+\partial^{2} / \partial y^{2}$ is the two-dimensional Laplacian operator; $J(a, b)=(\partial a / \partial x)(\partial b / \partial y)-(\partial a / \partial y)(\partial b / \partial x) ; f_{0}$ and $\beta_{0}$ are the Coriolis parameter and its meridional gradient at a given reference latitude $\varphi_{0}$, respectively; $N$ is the reference-state Brunt-Väisälä frequency and $\rho_{0}$ is the reference state density; $x, y, z$, and $t$ are the zonal, meridional, height, and time coordinates respectively; $\Gamma_{T}$ is the PV source; and $D_{T}$ is the dissipation as the PV sink.

Let us assume that the blocking disturbance is embedded in a basic flow $\psi_{\infty}(x, y, z)$ in the far field where all the blocking disturbances vanish. In this case, we may assume $\psi_{T}=\psi_{\infty}(x, y, z)+\psi(x, y, z, t), q_{T}=q_{\infty}(x, y, z)+$ $q(x, y, z, t), \Gamma_{T}=\Gamma_{\infty}+\Gamma$, and $D_{T}=D_{\infty}+D$, where $\Gamma_{\infty}(\Gamma)$ and $D_{\infty}(D)$ are the far-field (disturbance) PV source and dissipation, respectively. Under the assumption $J\left(\psi_{\infty}, q_{\infty}\right)=\Gamma_{\infty}-D_{\infty}$, from Eq. (1) one can obtain 


$$
\frac{\partial q}{\partial t}+J\left(\psi_{\infty}, q\right)+J\left(\psi, q_{\infty}\right)+J(\psi, q)=\Gamma-D,
$$

where $q_{\infty}(x, y, z)=f_{0}+\beta_{0} y+\nabla^{2} \psi_{\infty}+\left(1 / \rho_{0}\right)(\partial / \partial z)\left[\rho_{0}\left(f_{0}^{2} / N^{2}\right)\right.$ $\left.\left(\partial \psi_{\infty} / \partial z\right)\right] \quad$ and $q(x, y, z, t)=\nabla^{2} \psi+\left(1 / \rho_{0}\right)(\partial / \partial z)$ $\left[\rho_{0}\left(f_{0}^{2} / N^{2}\right)(\partial \psi / \partial z)\right]$.

Considering $U=-\partial \psi_{\infty} / \partial y, V=\partial \psi_{\infty} / \partial x$, and $\partial U / \partial x+$ $\partial V / \partial y=0$, Eq. (2) can be rewritten as

$$
\begin{aligned}
\left(\frac{\partial}{\partial t}+U \frac{\partial}{\partial x}\right) q+J(\psi, q)+\mathrm{PV}_{y} \frac{\partial \psi}{\partial x}= & -V \frac{\partial q}{\partial y}+\mathrm{PV}_{x} \frac{\partial \psi}{\partial y} \\
& +\Gamma-D,
\end{aligned}
$$

where $\mathrm{PV}_{y}=\partial q_{\infty} / \partial y=\beta_{0}+\partial^{2} V / \partial x \partial y-\partial^{2} U / \partial y^{2}-\left(1 / \rho_{0}\right)$ $(\partial / \partial z)\left[\rho_{0}\left(f_{0}^{2} / N^{2}\right)(\partial U / \partial z)\right]$ and $\mathrm{PV}_{x}=\partial q_{\infty} / \partial x=\nabla^{2} V+$ $\left(1 / \rho_{0}\right)(\partial / \partial z)\left[\rho_{0}\left(f_{0}^{2} / N^{2}\right)(\partial V / \partial z)\right]$.

Here, we suppose $\psi=\phi(z) \tilde{\psi}(x, y, t)$ as in Haines and Marshall (1987). If we further assume that $U, V, \mathrm{PV}_{y}$, and $\mathrm{PV}_{x}$ are slowly varying, then the following nondimensional PV disturbance equation, scaled by the characteristic velocity $\tilde{U}\left(\sim 10 \mathrm{~m} \mathrm{~s}^{-1}\right)$, length $\tilde{L}\left(\sim 10^{6} \mathrm{~m}\right)$, and height $\tilde{H}\left(\sim 10^{4} \mathrm{~m}\right)$, can be obtained by multiplying Eq. (3) with nondimensional $\phi(z)$ and integrating it from 0 to 1 in the vertical direction:

$$
\begin{aligned}
\left(\frac{\partial}{\partial t}+U \frac{\partial}{\partial x}\right) \tilde{q}+\Theta J(\tilde{\psi}, \tilde{q})+\mathrm{PV}_{y} \frac{\partial \tilde{\psi}}{\partial x}= & -V \frac{\partial \tilde{q}}{\partial y}+\mathrm{PV}_{x} \frac{\partial \tilde{\psi}}{\partial y} \\
& +\tilde{\Gamma}-\tilde{D}
\end{aligned}
$$

where $\mathrm{PV}_{y}=\beta+(\partial / \partial y)(\partial V / \partial x-\partial U / \partial y)-\left(1 / \rho_{0}\right)(\partial / \partial z)$ $\left\{\rho_{0}\left[\left(\tilde{L}^{2} f_{0}^{2}\right) /\left(\tilde{H}^{2} N^{2}\right)\right](\partial U / \partial z)\right\}, \beta=\beta_{0} \tilde{L}^{2} / \tilde{U}, \mathrm{PV}_{x}=\nabla^{2} V+$ $\left(1 / \rho_{0}\right)(\partial / \partial z)\left\{\rho_{0}\left[\left(\tilde{L}^{2} f_{0}^{2}\right) /\left(\tilde{H}^{2} N^{2}\right)\right](\partial V / \partial z)\right\}, \Theta=\int_{0}^{1} \phi(z)^{3} d z /$ $\int_{0}^{1} \phi(z)^{2} d z, \tilde{\Gamma}=\left(\tilde{L}^{2} / \tilde{U}^{2}\right) \int_{0}^{1} \Gamma \phi d z / \int_{0}^{1} \phi^{2} d z, \quad \tilde{D}=\left(\tilde{L}^{2} / \tilde{U}^{2}\right)$ $\int_{0}^{1} D \phi d z / \int_{0}^{1} \phi^{2} d z, \quad \tilde{q}(x, y, t)=\nabla^{2} \tilde{\psi}-F \tilde{\psi}, \quad$ and $F=$ $-\int_{0}^{1}\left(\left(1 / \rho_{0}\right)(d / d z)\left\{\rho_{0}\left[\left(\tilde{L}^{2} f_{0}^{2}\right) /\left(\tilde{H}^{2} N^{2}\right)\right](d \phi / d z)\right\} \phi d z\right) / \int_{0}^{1} \phi^{2}$ $d z$. For simplicity, we assume $\rho_{0}=\rho_{s} e^{-z}\left[\rho_{s}\right.$ is the atmospheric density at Earth's surface $(z=0)]$ in a nondimensional vertical domain $(0,1)$ and $N^{2}$ is a constant to consider the effect of the horizontal (mainly meridional) and vertical shears of the background westerly wind $(\mathrm{BWW})$ on blocking. As a result, it is easy to obtain $\mathrm{PV}_{y}=\beta+V_{x y}-U_{y y}+F_{r} U_{z_{1}}-F_{r} U_{z z}, \mathrm{PV}_{x}=\nabla^{2} V-$ $F_{r} V_{z}+F_{r} V_{z z}$ and $F=-F_{r}\left[\int_{0}^{1}\left(d^{2} \phi / d z^{2}\right) \phi d z\right] / \int_{0}^{1} \phi^{2} d z$, where $U_{y y}=\partial^{2} U / \partial y^{2}, V_{x y}=\partial^{2} V / \partial x \partial y, U_{z}=\partial U / \partial z, U_{z z}=$ $\partial^{2} U / \partial z^{2}, V_{z}=\partial V / \partial z, V_{z z}=\partial^{2} V / \partial z^{2}, F_{r}=\left(\tilde{L} / R_{d}\right)^{2}$, and $R_{d}=\tilde{H} N / f_{0}$ is the radius of Rossby deformation.

Since the blocking flow is often driven by synoptic-scale eddies, it is useful to separate the nondimensional disturbance streamfunction $\tilde{\psi}$ into planetary- and synopticscale components: $\tilde{\psi}=\psi+\psi^{\prime}$, where $\psi$ represents the planetary-scale blocking anomaly with a zonal wavenumber $k$ and $\psi^{\prime}$ denotes the synoptic-scale eddies with zonal wavenumbers $\tilde{k}_{j}(j=1,2, \ldots)$.
As in Luo (2000, 2005) and Luo et al. (2014, 2019), under the zonal scale separation assumption $k \ll \tilde{k}_{j}$, the nondimensional PV equations of the planetary-tosynoptic-scale interaction during the blocking life cycle can be expressed as

$$
\begin{aligned}
\left(\frac{\partial}{\partial t}+U \frac{\partial}{\partial x}\right) q+\Theta J(\psi, q)+\mathrm{PV}_{y} \frac{\partial \psi}{\partial x}= & -\Theta \nabla \cdot\left(\mathbf{v}^{\prime} q^{\prime}\right)_{P} \\
& -V \frac{\partial q}{\partial y}+\mathrm{PV}_{x} \frac{\partial \psi}{\partial y},
\end{aligned}
$$

$$
\begin{aligned}
\left(\frac{\partial}{\partial t}+U \frac{\partial}{\partial x}\right) q^{\prime}+\mathrm{PV}_{y} \frac{\partial \psi^{\prime}}{\partial x}= & -\Theta J\left(\psi^{\prime}, q\right)-\Theta J\left(\psi, q^{\prime}\right) \\
& -V \frac{\partial q^{\prime}}{\partial y}+\mathrm{PV}_{x} \frac{\partial \psi^{\prime}}{\partial y}+\nabla^{2} \psi_{S}^{*},
\end{aligned}
$$

where $q(x, y, t)=\nabla^{2} \psi-F \psi, q^{\prime}(x, y, t)=\nabla^{2} \psi^{\prime}-F \psi^{\prime}$, and $\mathbf{v}^{\prime}=\left(-\partial \psi^{\prime} / \partial y, \partial \psi^{\prime} / \partial x\right)$ is the horizontal wind vector of synoptic-scale eddies. In the derivation of Eq. (5), $\tilde{\Gamma}-\tilde{D}=$ $\nabla^{2} \psi_{S}^{*}$ has been assumed to represent a synoptic-scale PV source as a synoptic-scale wavemaker (Shutts 1983) that excites and maintains preexisting synoptic-scale eddies in the upstream side of incipient blocking (Luo 2005). In Eq. (5a), the subscript $P$ of $-\nabla \cdot\left(\mathbf{v}^{\prime} q^{\prime}\right)_{P}$ denotes that $-\nabla \cdot\left(\mathbf{v}^{\prime} q^{\prime}\right)$ has the same spatial structure as that of the blocking flow.

In the vertical direction, any nondimensional vertical function $\phi(z)$ of the blocking and synoptic-scale eddy components can be expanded as $\phi(z)=c_{0}+$ $\sum_{n=1}^{\infty} c_{n} \sin n \pi z$ in the vertical domain $(0,1)$, where $c_{0}$ and $c_{n}$ are the constants. When $c_{n}=0$ and $c_{0}$ is a constant, one can have $\phi(z)=c_{0}$. In this case, the planetary- and synoptic-scale anomalies possess a barotropic structure without vertical variation. When $c_{0} \neq 0, c_{1} \neq 0$, and $c_{n}=$ 0 for $n \geq 2$, the blocking anomaly shows a vertical variation, thus reflecting that the blocking and synopticscale anomalies can have a baroclinic structure. Crudely speaking, the observed blocking anomaly has a maximum amplitude in the middle and upper tropospheric layers near the 500-300-hPa levels. For this reason, we consider two cases: 1) $c_{0}=1$ and $c_{n}=0$ for $n \geq 1$ (barotropic case) and 2) $c_{0}=1$ and $c_{n}=Z_{b} \neq 0$ for $n=1$ (baroclinic structure). On the other hand, because $U$ and $V$ have horizontal and vertical shears or because $\mathrm{PV}_{y}$ and $\mathrm{PV}_{x}$ include the horizontal and vertical shears of the background horizontal winds and basic atmospheric stratification, using Eq. (5) can allow us to examine the barotropic and baroclinic effects of the basic-state flow on eddy-driven blocking. Thus, our present model is an extension of the NMI model of eddy-driven blocking wave packet proposed by Luo $(2000,2005)$ and Luo et al. $(2014,2018,2019)$ and Zhang and Luo (2020). 


\section{Analytical solution of the extended NMI model}

As used in Luo et al. $(2018,2019)$ and Zhang and Luo (2020), Eq. (5) can be analytically solved by assuming $\psi^{\prime}=\psi_{1}^{\prime}+\psi_{2}^{\prime}$ that is composed of preexisting eddies $\psi_{1}^{\prime}$ and deformed eddies $\psi_{2}^{\prime}$ during the blocking life cycle. Here, $\psi_{1}^{\prime}$ represents preexisting incident synoptic-scale eddies with two zonal wavenumbers $\tilde{k}_{1}$ and $\tilde{k}_{2}$ corresponding to the frequencies of $\tilde{\omega}_{1}$ and $\tilde{\omega}_{2}$, respectively, whereas $\psi_{2}^{\prime}$ denotes deformed eddies due to the feedback of intensified blocking on preexisting eddies and represents the eddy straining or CWB during the blocking episode.

Equation (5a) can also be rewritten as $\partial q / \partial t+J(\psi$, $\Theta q+\mathrm{PV})+J\left(\psi_{\infty}, q\right)=-\Theta \nabla \cdot\left(\mathbf{v}^{\prime} q^{\prime}\right)_{P}$, where $\mathrm{PV}=q_{\infty}(x$, $y, z)$ and $\psi_{\infty}$ is the nondimensional three-dimensional basic-state streamfunction. Under the assumption $J(\psi$, $\Theta q+\mathrm{PV})+J\left(\psi_{\infty}, q\right) \approx 0$ as in Luo et al. (2014) because of approximate linear relationship between $q$ and $\psi$ in a slowly varying background flow, one can get $\partial q / \partial t \approx$ $-\Theta \nabla \cdot\left(\mathbf{v}^{\prime} q^{\prime}\right)_{P}$. Because $\psi_{2}^{\prime}$ is very weak during the initiation stage $(t \sim 0)$ of blocking, one can easily obtain $\partial q / \partial t \approx-\Theta \nabla \cdot\left(\mathbf{v}_{1}^{\prime} q_{1}^{\prime}\right)_{P}$. Thus, it is inevitable to infer that the preexisting incident synoptic-scale eddies $\psi_{1}^{\prime}$ can amplify an incipient block into a typical blocking under the forcing of $-\nabla \cdot\left(\mathbf{v}_{1}^{\prime} q_{1}^{\prime}\right)_{P}$, when $-\nabla \cdot\left(\mathbf{v}_{1}^{\prime} q_{1}^{\prime}\right)_{P}$ matches the PV anomaly $q$ of the downstream incipient block. This mechanism is the so-called eddy-blocking matching mechanism of blocking proposed by Luo et al. (2014, 2019). The incipient block, as denoted by a preexisting planetary-scale ridge, was also found to be related to the downward propagation of stratospheric planetary waves (Kodera et al. 2013) and other processes. However, it must be emphasized in this theoretical model that the eddy straining or CWB is unimportant for the blocking intensification and decay because $-\nabla \cdot\left(\mathbf{v}_{1}^{\prime} q_{1}^{\prime}\right)_{P}$ does not include the effect of deformed eddies $\psi_{2}^{\prime}$, as demonstrated in Luo $(2000,2005)$ and Luo et al. $(2014,2019)$. This viewpoint contradicts the eddy straining theory of blocking proposed by Shutts (1983).

To analytically solve Eq. (5), here we further make some assumptions that $\psi_{\infty}(x, y, z)$ is slowly varying in space compared to the blocking anomaly and the meridional background wind $V$ is much weaker than the background zonal wind $U$ as observed in Luo et al. (2018). Using the Wentzel-Kramers-Brillouin (WKB) method as in Luo (2005), the approximate analytical solution of the nondimensional total streamfunction field $\psi_{T}$ of blocking from Eq. (5) in a $\beta$-plane channel with a nondimensional width of $L_{y}$ can be obtained in fast variable form of

$$
\begin{aligned}
\psi_{T}= & \psi_{\infty}(x, y, z)+\phi(z)\left(\psi+\psi^{\prime}\right) \approx \psi_{P}+\psi_{e}^{\prime}, \\
\psi_{P}= & \psi_{\infty}(x, y, z)+\phi(z) \psi \approx-\int_{0}^{y} U\left(x, y^{\prime}, z\right) d y^{\prime} \\
& +\int_{-L_{x}}^{x} V\left(x^{\prime}, y, z\right) d x^{\prime}+\psi_{B}+\psi_{m}, \\
\psi_{B}= & \phi(z) B \sqrt{\frac{2}{L_{y}}} \exp [i(k x-\omega t)] \sin m y+\mathrm{cc}, \\
\psi_{m}= & -\phi(z) \Theta|B|^{2} \sum_{n=1}^{\infty} q_{n} g_{n} \cos (n+1 / 2) m y, \\
\psi_{e}^{\prime}= & \phi(z) \psi^{\prime} \approx \phi(z)\left(\psi_{1}^{\prime}+\psi_{2}^{\prime}\right)=\psi_{e 1}^{\prime}+\psi_{e 2}^{\prime}, \\
\psi_{e 1}^{\prime}= & \phi(z) \psi_{1}^{\prime}=\phi(z) f_{0}(x)\left\{\alpha_{1} \exp \left[i\left(\tilde{k}_{1} x-\tilde{\omega}_{1} t\right)\right]\right. \\
& \left.+\alpha_{2} \exp \left[i\left(\tilde{k}_{2} x-\tilde{\omega}_{2} t\right)\right]\right\} \sin \left(\frac{m}{2} y\right)+\mathrm{cc},
\end{aligned}
$$

$$
\begin{aligned}
& i\left(\frac{\partial B}{\partial t}+C_{g} \frac{\partial B}{\partial x}\right)+\lambda \frac{\partial^{2} B}{\partial x^{2}}+\delta|B|^{2} B+G f_{0}^{2} \\
& \quad \times \exp [-i(\Delta k x+\Delta \omega t)]=0
\end{aligned}
$$

where $\omega=U k-\left(\mathrm{PV}_{y} k\right) /\left(k^{2}+m^{2}+F\right), \tilde{\omega}_{j}=$ $U \tilde{k}_{j}-\left(\mathrm{PV}_{y} \tilde{k}_{j}\right) /\left(\tilde{k}_{j}^{2}+m^{2} / 4+F\right)(j=1,2), \alpha_{1}=1, \alpha_{2}=$ $\alpha=-1, C_{g}=\partial \omega / \partial k=U-\left[\mathrm{PV}_{y}\left(m^{2}+F-k^{2}\right)\right] /\left(k^{2}+\right.$ $\left.m^{2}+F\right)^{2}, \Delta k=k-\left(\tilde{k}_{2}-\tilde{k}_{1}\right), \Delta \omega=\tilde{\omega}_{2}-\tilde{\omega}_{1}-\omega$ is the difference between the frequency $\tilde{\omega}_{2}-\tilde{\omega}_{1}$ of the preexisting eddy forcing and the frequency $\omega$ of the blocking carrier wave, which reflects the forcing of the blocking dipole by preexisting synoptic-scale eddies, and cc denotes the complex conjugate of its preceding term. One can have $\Delta \omega \approx \tilde{\omega}_{2}-\tilde{\omega}_{1}$ for $\omega \sim 0$ in Eq. ( $\left.6 \mathrm{~h}\right)$. 
Also note that $|B|^{2}=B B^{*}$ for $B^{*}$ being the complex conjugate of the envelope amplitude $B$ of a nonlinear blocking wave packet, $\lambda=\left[3\left(m^{2}+F\right)-k^{2}\right] \mathrm{PV}_{y} k /\left(k^{2}+\right.$ $\left.m^{2}+F\right)^{3}$ is the linear dispersion strength, $\delta=\Theta^{2} \delta_{B}$ is the nonlinearity strength, $\delta_{B}=\delta_{N} / \mathrm{PV}_{y}, G=\Theta G_{B}, q_{n}=$ $q_{N n} / \mathrm{PV}_{y}, m=-2 \pi / L_{y}, k=2 k_{0}, k_{0}=1 /\left(6.371 \cos \varphi_{0}\right)$, $\tilde{k}_{j}=n_{j} k_{0}(j=1,2), n_{j}$ is the positive integer (e.g., $n_{1}=9$ and $n_{2}=11$ used in this paper), $f_{0}(x)=a_{0} e^{-\mu \varepsilon^{2}\left(x+x_{T}\right)^{2}}$ is the slowly varying eddy amplitude distribution located at $x=-x_{T}, \varepsilon$ is a small parameter, $a_{0}$ is the constant maximum eddy intensity, and $\mu>0$. Clearly, the linear dispersion strength is proportional to the background meridional $\mathrm{PV}$ gradient $\left(\mathrm{PV}_{y}\right)$, whereas the nonlinearity strength is proportional to the inverse of $\mathrm{PV}_{y}$. Thus, there is an inverse ratio rule of the linear dispersionnonlinearity relation for a blocking event in a threedimensional baroclinic flow. Here, $p_{j}, r_{j}, s_{j}, h_{j}$, and $Q_{j}$ in Eq. $(6 \mathrm{~g})$ are given in appendix A, whereas the coefficients $\delta_{N}, q_{N n}, g_{n}$, and $G_{B}$ of the amplitude Eq. (6h) of blocking wave packet are defined in appendix B.

In Eq. (6), the total streamfunction field $\psi_{T}$ of eddydriven blocking flow consists of planetary- and synopticscale parts $\left(\psi_{P}\right.$ and $\left.\psi_{e}^{\prime}\right)$, in which $\psi_{P}$ represents the planetary-scale field of blocking, and $\psi_{e}^{\prime}$ denotes the synoptic-scale anomaly field with the effect of $\phi(z)$ during the blocking life cycle. In $\psi_{P}, \psi_{B}$ is the blocking wavy anomaly with the removal of $\psi_{\infty}(x, y, z)$ and $\psi_{m}$ from $\psi_{P}$, whereas $\psi_{m}$ represents the change of the mean zonal wind due to the feedback of intensified blocking wavy anomaly $\psi_{B}$ during the blocking life cycle. Since $\phi(z)$ and $\psi_{1}^{\prime}$ of $\psi_{e}^{\prime}=\phi(z) \psi^{\prime} \approx \phi(z)\left(\psi_{1}^{\prime}+\psi_{2}^{\prime}\right)$ are prespecified in Eq. (6e), the spatiotemporal evolution of synoptic-scale eddies $\psi_{e}^{\prime}$ is mainly determined by the deformed eddies $\psi_{2}^{\prime}$. In $\psi^{\prime}=\psi_{1}^{\prime}+\psi_{2}^{\prime}$, the deformed eddies $\psi_{2}^{\prime}$ depends on the evolution of the blocking wavy anomaly $\psi_{B}$ because $\psi_{2}^{\prime}$ includes the blocking envelope amplitude $B$, whereas $\psi_{1}^{\prime}$ represents preexisting synoptic-scale eddies that are located in the upstream side of the incipient blocking. Because $\psi_{2}^{\prime}$ vanishes in the absence of blocking and is weak when the blocking amplitude is small, the deformed eddies $\psi_{2}^{\prime}$ may be regarded as a concomitant phenomenon of intensified blocking (Luo 2000, 2005), though the eddy straining and CWB can be reflected by the deformed eddies $\psi_{2}^{\prime}$.

Since the preexisting eddy forcing $-\nabla \cdot\left(\mathbf{v}_{1}^{\prime} q_{1}^{\prime}\right)_{P}$ is caused by preexisting synoptic-scale eddies $\psi_{1}^{\prime}$ rather than deformed eddies $\psi_{2}^{\prime}$ (eddy straining or CWB), our NMI model suggests that the eddy straining mechanism of Shutts (1983) or CWB does not work during the intensification and maintenance of blocking (Luo 2000, 2005; Luo et al. 2014, 2019). In this paper, $-\nabla \cdot\left(\mathbf{v}_{1}^{\prime} q_{1}^{\prime}\right)_{P}$ has been assumed to match the incipient block (or a preexisting small amplitude block) in order for it to grow. Under the forcing of $-\nabla \cdot\left(\mathbf{v}_{1}^{\prime} q_{1}^{\prime}\right)_{P}$, the evolution of a small amplitude incipient block into a typical blocking flow was thought of as being a nonlinear initial value problem (Luo 2000, 2005). In addition, the spatiotemporal variation of eddy-driven dipole blocking, as denoted by the blocking wavy anomaly $\psi_{B}$, is also considered as the evolution of a nonlinear wave packet in the form of $B \exp [i(k x-\omega t)]$, which is described by a nonlinear Schrödinger (NLS) equation with a preexisting eddy forcing (Luo 2000, 2005; Luo et al. 2014, 2019; Zhang and Luo 2020).

Here, we have extended our previous nonlinear wave packet theory to include a general three-dimensional basic flow to examine the effect of the basic flow with horizontal and vertical shears on eddy-driven blocking, even though the evolution of blocking depends on the initial value of preexisting planetary-scale wave and synoptic-scale eddies. As an example, we consider a barotropic case and show a schematic diagram in Fig. 1 to depict how preexisting upstream synoptic-scale eddies create a blocking from an incipient block through the forcing of $-\nabla \cdot\left(\mathbf{v}_{1}^{\prime} q_{1}^{\prime}\right)_{P}$ and how the background flow change influences the evolution of eddy-driven blocking. Here, our basic idea on the blocking generation and its evolution is different from the mechanism of Shutts (1983), who emphasized the importance of eddy straining in the blocking maintenance. However, in our NMI model, the preexisting synoptic-scale eddies are shown to play a key role in the blocking intensification and decay, whereas the eddy straining is only a result of the blocking intensification and maintenance. To further examine this problem, we fix the parameters of the incipient block and preexisting synoptic-scale eddies prior to the blocking onset as shown in Table 1, but vary the basic-flow parameters.

When $c_{0}=1$ and $c_{n}=0$ for $n \geq 1$ are allowed, there are $\phi(z)=1$ and $\Theta=1$. In this case, we have $F=0$. However, when there is $\phi(z)=1+Z_{b} \sin \pi z$ for $c_{0}=1$, $c_{1}=Z_{b}$ and $c_{n}=0(n \geq 2)$, we can obtain $F=F_{r} Z_{b} \pi^{2}$ $\left(2 / \pi+Z_{b} / 2\right) /\left(1+4 Z_{b} / \pi+Z_{b}^{2} / 2\right)$ and $\Theta=\left[1+6 Z_{b} / \pi+\right.$ $\left.3 Z_{b}^{2} / 2+4 Z_{b}^{3} /(3 \pi)\right] /\left(1+4 Z_{b} / \pi+Z_{b}^{2} / 2\right)$. A special case of $F=0$ and $\Theta=1$ is found for $Z_{b}=0$ (a barotropic case with no vertical variation). In this case, we further have $\mathrm{PV}_{y}=\beta+V_{x y}-U_{y y}$ and $\mathrm{PV}_{x}=\nabla^{2} V$ for a purely barotropic background horizontal wind with $U=U(x, y)$, $V=V(x, y)$, and $\phi(z)=1$. As a result, Eq. (6) reduces to the NMI model in a zonally varying barotropic basic flow presented by Luo et al. (2019) and Zhang and Luo (2020). For $Z_{b}>0$, there is $\Theta>1$. For example, we have $\Theta=1.13$ for $Z_{b}=0.2$. This reflects that the presence of $\Theta>1$ tends to enhance the nonlinearity of blocking system due to $\delta=$ $\Theta^{2} \delta_{B}>\delta_{B}$ and strengthen the preexisting eddy forcing because of $G=\Theta G_{B}>G_{B}$. Thus, in some degree the baroclinic structure of blocking tends to favor the maintenance itself because of increased nonlinearity. 
Preexisting eddies $\psi_{1}^{\prime}$ Incipient block

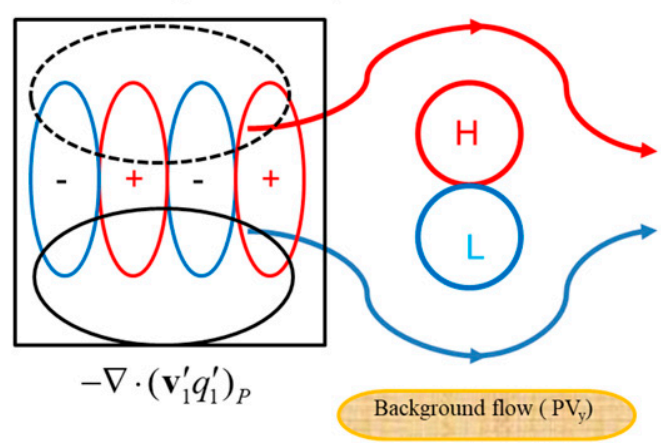

Eddy evolution Blocking formation

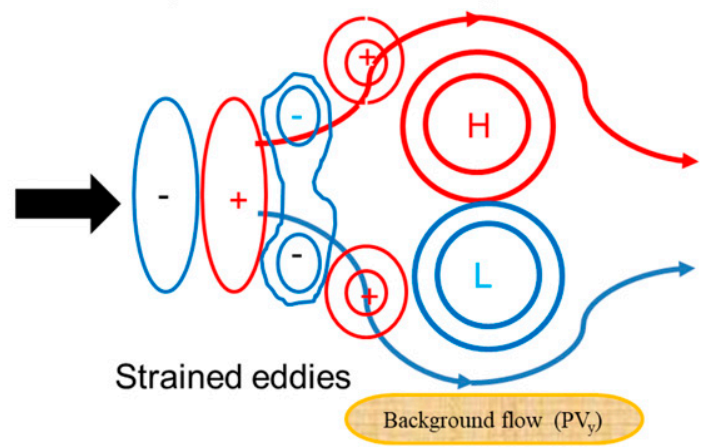

FIG. 1. Schematic diagram of preexisting upstream synoptic-scale eddies $\psi_{1}^{\prime}$ leading to (right) the blocking formation from (left) an incipient dipole block in a background flow, as denoted by the meridional gradient $\left(\mathrm{PV}_{\mathrm{y}}\right)$ of background potential vorticity, through the forcing of $-\nabla \cdot\left(\mathbf{v}_{1}^{\prime} q_{1}^{\prime}\right)_{P}$ caused by preexisting eddies $\psi_{1}^{\prime}$ in the extended NMI model as a nonlinear initial value problem. When $-\nabla \cdot\left(\mathbf{v}_{1}^{\prime} q_{1}^{\prime}\right)_{P}$ leads to the blocking formation, the preexisting eddies are deformed and strained to result in (right) an eddy straining or cyclonic wave breaking due to the feedback of intensified blocking. When $\mathrm{PV}_{y}$ is small within the blocking region, the region of the preexisting incipient block is a weak dispersion or nondispersion and strong nonlinearity media region that favors the maintenance of eddy-driven blocking. At the same time, when the preexisting upstream synoptic eddies move eastward and enter a small $\mathrm{PV}_{y}$ region, $-\nabla \cdot\left(\mathbf{v}_{1}^{\prime} q_{1}^{\prime}\right)_{P}$ has a long duration and then leads to a long-lived blocking. As a result, the background flow change can influence the evolution (strength, movement, and duration) of eddy-driven blocking. Here, $\mathrm{H}$ (L) denotes the anticyclonic (cyclonic) anomaly of blocking, $+(-)$ represents the synoptic-scale anticyclone (cyclone), and the anticyclonic (cyclonic) forcing of $-\nabla \cdot\left(\mathbf{v}_{1}^{\prime} q_{1}^{\prime}\right)_{P}$ is marked by the black dashed (solid) oval in the black box on the left side of the figure.

Because $\mathrm{PV}_{y}$ includes the horizontal and vertical shears of the background flow, the nonlinear behavior (duration, strength, and movement) of eddy-driven blocking is inevitably influenced by the horizontal and vertical shears of background basic flow.

It is useful to divide $\mathrm{PV}_{y}$ into $\mathrm{PV}_{y}=\beta+\mathrm{PV}_{y}^{D}+\mathrm{PV}_{y}^{T}$, where $\mathrm{PV}_{y}^{D}=\partial^{2} V / \partial x \partial y-\partial^{2} U / \partial y^{2}$ denotes the barotropic effect (horizontal shear) of the basic flow and $\mathrm{PV}_{y}^{T}=F_{r} \partial U / \partial z-F_{r} \partial^{2} U / \partial z^{2}$ represents its baroclinic effect (vertical shear of the basic flow). It is found that when $\mathrm{PV}_{y}$ is small, the linear dispersion $\lambda$ is weak and the nonlinearity strength $\delta$ is strong in the nonlinear wave packet Eq. (6h), thus indicating that the blocking can last for a long time. When one assumes $U=U_{B}(x, y)+$ $U_{T}(z)$ and $V=V_{B}(x, y)+V_{T}(z)$, where $U_{B}(x, y)$ or $V_{B}(x, y)$ and $U_{T}(z)$ or $V_{T}(z)$ are the barotropic and baroclinic parts of the basic flow, respectively, it is easy to obtain $\mathrm{PV}_{y}^{D}=\partial^{2} V_{B} / \partial x \partial y-\partial^{2} U_{B} / \partial y^{2}$ and $\mathrm{PV}_{y}^{T}=$ $F_{r} \partial U_{T} / \partial z-F_{r} \partial^{2} U_{T} / \partial z^{2}$. Because $\partial U_{T} / \partial z \propto-\partial \bar{T} / \partial y(\partial \bar{T} / \partial y$ is the meridional gradient of background air temperature

TABLE 1. Values of given parameters in the extended NMI model.

\begin{tabular}{lc}
\hline \hline \multicolumn{1}{c}{ Parameters } & Value \\
\hline Nondimensional zonal wavenumber $k$ of the initial blocking wavy anomaly & $k=2 /\left(6.371 \cos \varphi_{0}\right)$ \\
Nondimensional zonal wavenumber $\tilde{k}_{1}$ of preexisting synoptic-scale eddies & $\tilde{k}_{1}=9 /\left(6.371 \cos \varphi_{0}\right)$ \\
Nondimensional zonal wavenumber $\tilde{k}_{2}$ of preexisting synoptic-scale eddies & $\tilde{k}_{2}=11 /\left(6.371 \cos \varphi_{0}\right)$ \\
Reference latitude $\varphi_{0}$ & $55^{\circ} \mathrm{N}$ \\
$\Omega$ in the Coriolis parameter $f_{0}=2 \Omega \sin \varphi_{0}$ & $7.29 \times 10^{-5} \mathrm{~s}^{-1}$ \\
Nondimensional width $L_{y}$ of the $\beta$ channel & 5 \\
Characteristic horizontal length $\tilde{L}$ & $10^{6} \mathrm{~m}$ \\
Characteristic vertical length $\tilde{H}$ & $10^{4} \mathrm{~m}$ \\
Characteristic horizontal wind speed $\tilde{U}$ & $10 \mathrm{~m} \mathrm{~s}^{-1}$ \\
Atmospheric state stratification $N^{2}$ & $2 \times 10^{-4} \mathrm{~s}^{-2}$ \\
& 0.72 \\
$\left.F_{r}=\left(\tilde{L} f_{0}\right)^{2} N\right)^{2}$ & 1.2 \\
$\mu$ & $2.87 / 2$ \\
Zonal location $x_{T}$ of preexisting upstream synoptic-scale eddies & 0.12 \\
Amplitude of the initial blocking & 0.3 \\
Small parameter $\varepsilon$ & 0.24 \\
\hline
\end{tabular}


$\bar{T}), \mathrm{PV}_{y}^{T}$ actually represents the effect of the meridional temperature gradient (MTG), which reflects the thermodynamic effect of the basic flow on blocking and thus is referred to as a thermodynamic PV gradient. On the other hand, because $\mathrm{PV}_{y}^{D}$ as a dynamical PV gradient reflects the dynamical effect of the basic flow, one can evaluate the dynamical and thermodynamic effects of the basic flow on the nonlinear behavior of eddy-driven blocking by calculating the magnitude and distribution of $\mathrm{PV}_{y}^{D}$ and $\mathrm{PV}_{y}^{T}$. Thus, it is thought that Eq. (6) is an extension of the NMI model of eddy-driven blocking wave packet in the barotropic atmosphere derived previously by Luo et al. $(2018,2019)$ and Zhang and Luo (2020). This extended NMI model provides a useful tool for examining how the barotropic and baroclinic (or MTG) components of a background flow influence the spatiotemporal evolution of eddy-driven blocking.

When the background wind is purely zonal and $U=u_{0}$ is a constant, one can have $\mathrm{PV}_{y}=\beta$. The mathematical form of $\mathrm{PV}_{y}$ is different from the result of $\mathrm{PV}_{y}=\beta+F u_{0}$ in a uniform westerly wind $u_{0}$ obtained by Luo et al. (2019) and Zhang and Luo (2020) when the divergence of horizontal winds is considered in a barotropic model as in Yeh (1949). Although the meridional and vertical variations of $B$ in $y$ and $z$ directions are not explicitly included in Eq. (6h), it essentially shows meridional and vertical variations in that $\mathrm{PV}_{y}$ and $U$ have been considered as being the functions of $y$ and $z$. For given initial blocking and parameter conditions, the high-order split-step Fourier scheme (Muslu and Erbay 2005) is used to solve Eq. (6h) to derive the spatiotemporal solution of the envelope amplitude $B$ of the blocking wave packet. In the split-step Fourier scheme calculation of Eq. (6h), the blocking wave packet has been assumed to be a superposition of many linear modes or waves (Luo et al. 2019). Moreover, we also use the periodic boundary conditions same as used in Luo et al. (2019).

In this paper, we consider a BWW $U=U(x, y, z)$ and $V \approx 0$ as a pure zonal basic flow. We refer to $U=U_{B}(x, y)$ as a barotropic BWW if $U=U_{B}(x, y)$ and $U_{T}(z)=0$ are assumed. Correspondingly, $U=U_{T}(z)$ is referred to as a baroclinic BWW if $U_{B}(x, y)=0$ is assumed. Because $\phi(z)=1+Z_{b} \sin \pi z$, the eddy-driven blocking may be called a "baroclinic (barotropic) blocking" with (without) vertical variation for $Z_{b}>0\left(Z_{b}=0\right)$. It is also noted that the barotropic blocking becomes a baroclinic blocking even under $Z_{b}=0$ and $F=0$, if the effect of $\mathrm{PV}_{y}^{T}=F_{r} U_{z}-F_{r} U_{z z}$ is considered in our extended NMI model. Such a blocking is still a baroclinic blocking in the presence of $U_{z}$ for $F_{r} \neq 0$. Thus, the evolution (duration, movement and strength) of blocking depends not only on the horizontal (mainly meridional) shear of the BWW (the value of $\mathrm{PV}_{y}^{D}=V_{x y}-U_{y y}$ ), but also on the vertical shear of the BWW and the strength of atmospheric stratification or $F_{r} \neq 0$ (the value of $\mathrm{PV}_{y}^{T}=$ $\left.F_{r} \partial U / \partial z-F_{r} \partial^{2} U / \partial z^{2}\right)$. One can also have $-\Delta \omega=$ $\mathrm{PV}_{y}\left[\tilde{k}_{2} /\left(\tilde{k}_{2}^{2}+m^{2} / 4+F\right)-\tilde{k}_{1} /\left(\tilde{k}_{1}^{2}+m^{2} / 4+F\right)-k /\left(k^{2}+\right.\right.$ $\left.m^{2}+F\right)$ ] for $F \neq 0$ in a baroclinic atmosphere with stratification. This formula shows that the frequency $\Delta \omega$ of the preexisting eddy forcing is smaller in a baroclinic blocking flow $(F \neq 0)$ than that in a barotropic blocking flow $\left[F=0\right.$ and $U=U_{B}(x, y)$ for $\left.U_{T}(z)=0\right]$. Thus, the preexisting eddy forcing as a driver of blocking has inevitably a longer duration for a baroclinic blocking flow than for a barotropic blocking flow, leading to a longlived baroclinic blocking.

\section{Theoretical results}

While Eq. (6) is the analytical solution of eddy-driven blocking flow, it is useful to present the spatiotemporal evolution solution of the blocking field in this section. In our calculations, we choose the parameters $B(x, y, z$, $0)=0.3, Z_{b}=0$, and $Z_{b}=0.2$, but allow the BWW to vary. The other fixed parameters $n_{1}=9, n_{2}=11, L_{y}=5, N^{2}=$ $2 \times 10^{-4} \mathrm{~s}^{-1}, \mu=1.2, \varepsilon=0.24, x_{T}=2.87 / 2, a_{0}=0.12$, and $\varphi_{0}=55^{\circ} \mathrm{N}$ are shown in Table 1 . For $Z_{b}=0.2$, we have $F=$ 0.82 . We also test the sensitivity of the obtained results to the background flow parameters. The obtained results are similar (not shown).

To see whether the background media where a blocking occurs changes with the atmospheric stratification, we show the variations of the linear phase speed $C_{p}$, linear group velocity $C_{g}$, linear dispersion term $\lambda$, nonlinearity strength $\delta$, the eddy forcing frequency $\Delta \omega$, eddy forcing coefficient $|G|, \delta / \lambda$, and $C_{g p}=C_{g}-C_{p}$ with the parameter $F$ in Fig. 2 for a uniform BWW with $U=$ 0.7 , when $F$ is considered as a changing parameter. It is found that while $C_{p}$ (Fig. 2a) and $C_{g}$ (Fig. 2b) increase with the increasing of $F, C_{g p}$ (Fig. 2h) decreases with the increasing of $F$, thus indicating that a weak energy dispersion can be seen in a large value range of $F$. Because $\delta / \lambda$ measures a relative magnitude between the nonlinearity strength $\delta$ and linear dispersion term $\lambda$, the value of $\delta / \lambda$ can reflect whether the value of $F$ changes the dispersion or nonlinearity of blocking system. We also see that $\delta / \lambda$ increases with the increasing of $F$ (Fig. 2g), though both $\lambda$ (Fig. 2c) and $\delta$ (Fig. 2d) decrease with $F$ and $\lambda$ has a larger decrease than $\delta$. Thus, it is concluded that a large value of $F$ favors the weak dispersion or strong nonlinearity of blocking.

On the other hand, because $\Delta \omega$ decreases with the increasing of $F$, the preexisting eddy forcing has a long duration in the region of large $F$. Thus, eddy-driven blocking is inevitably long-lived in a large $F$ region, which can also be explained by $C_{g p}=2 k^{2} \mathrm{PV}_{y} /\left(k^{2}+m^{2}+F\right)^{2}$ 
(a)

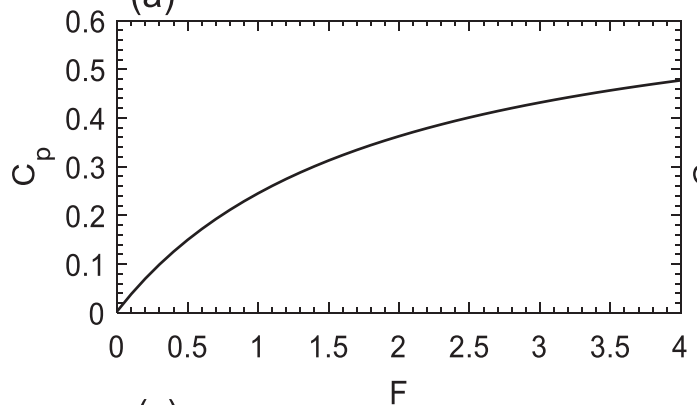

(c)

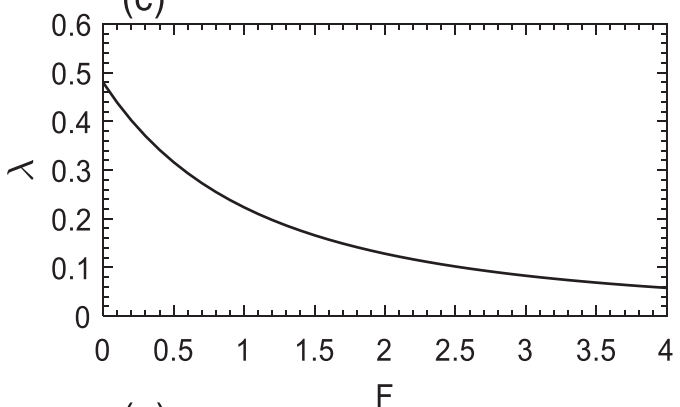

(e)

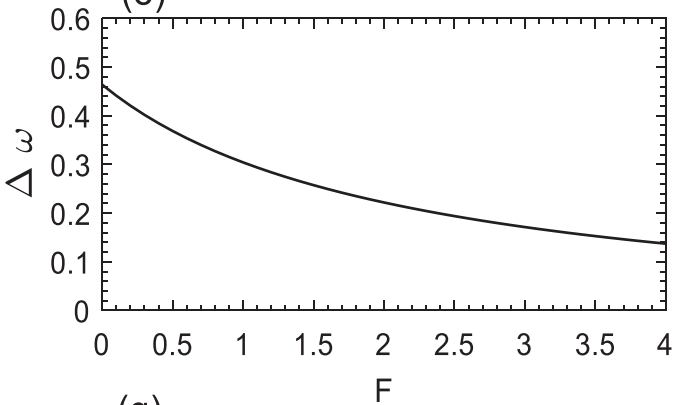

(g)

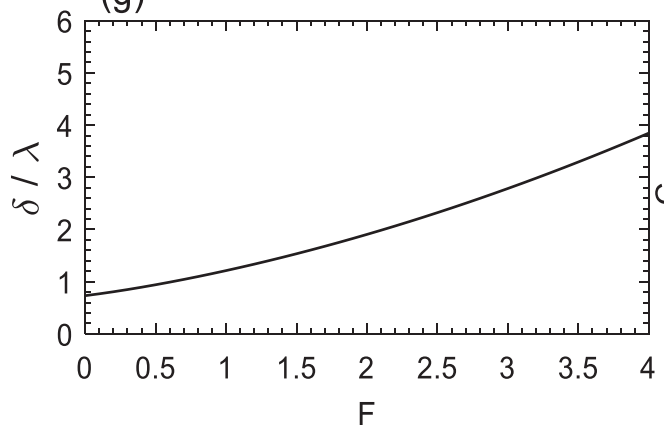

(b)

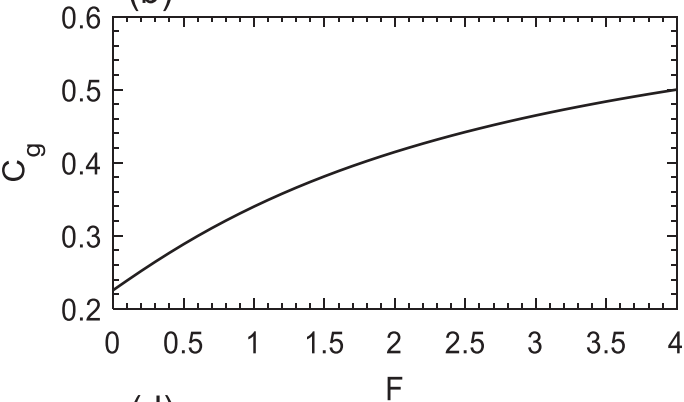

(d)

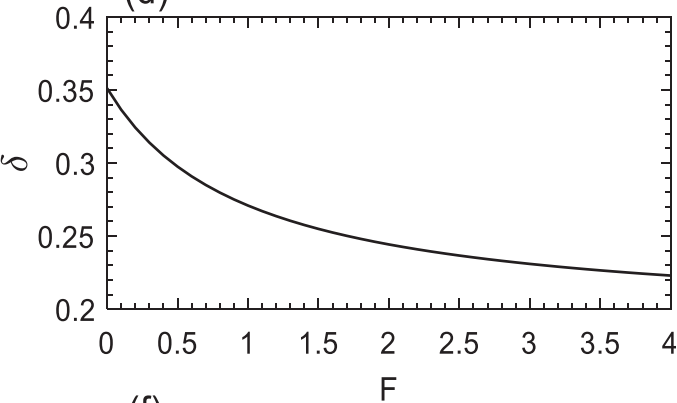

(f)

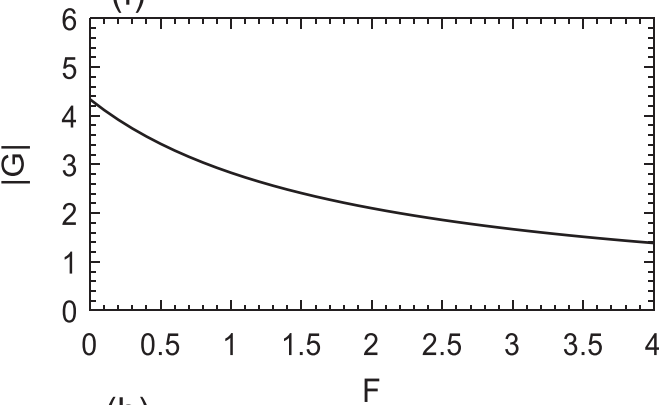

(h)

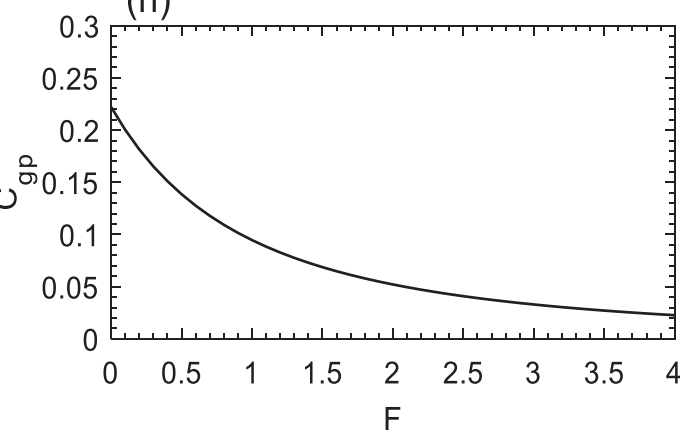

FIG. 2. Variations of (a) linear phase speed $C_{p}$, (b) group velocity $C_{g}$, (c) linear dispersion term $\lambda$, (d) nonlinearity strength $\delta$, (e) the eddy forcing frequency $\Delta \omega$, (f) eddy forcing coefficient $|G|$, (g) $\delta / \lambda$, and (h) $C_{g p}=C_{g}-C_{p}$ with the parameter $F$ for $\mathrm{PV}_{y}=\beta$ and $U=0.7$.

obtained from the linear theory. When $\mathrm{PV}_{y}$ is small or when $F$ is large, $C_{g p}$ is small. In this case, the energy dispersion of the linear Rossby wave packet is weak. The dispersion time scale of the Rossby wave packet is $T_{\beta}=$ $2 \pi / C_{g p} \sim\left(k^{2}+m^{2}+F\right)^{2} /\left(\mathrm{PV}_{y} k^{2}\right)$, which does not require that the zonal wavelength of synoptic-scale eddies must be small. Instead, it depends on the zonal wavelength of
Rossby wave packet and the magnitude of background $\mathrm{PV}_{y}$. When $k$ or $\mathrm{PV}_{y}$ is small, $T_{\beta}$ is large. As a result, the linear wave packet has a long dispersion time scale, corresponding to a weak energy dispersion. Naturally, the longer zonal scale of the Rossby wave packet and small $\mathrm{PV}_{y}$ tend to maintain the wave packet itself. This theory is different from that of Hoskins and James (2014), who concluded that 


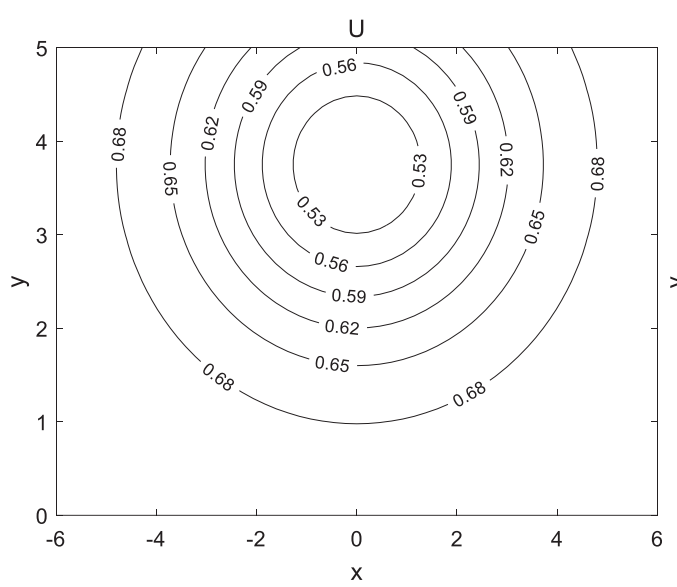

(a)

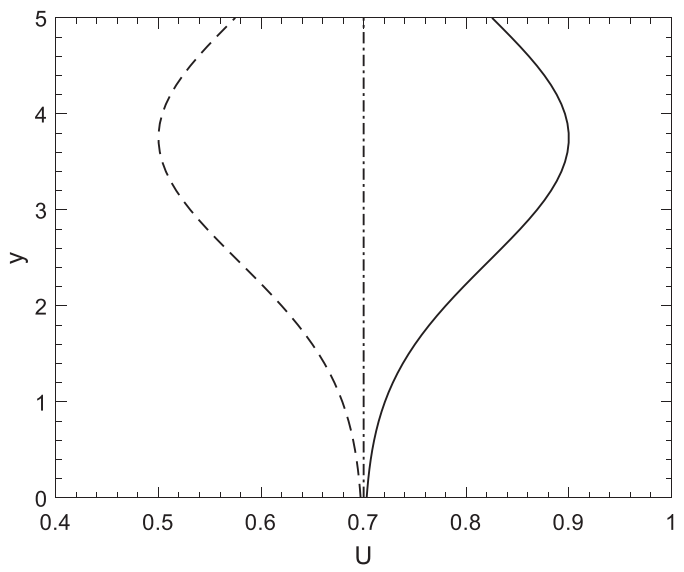

(c)

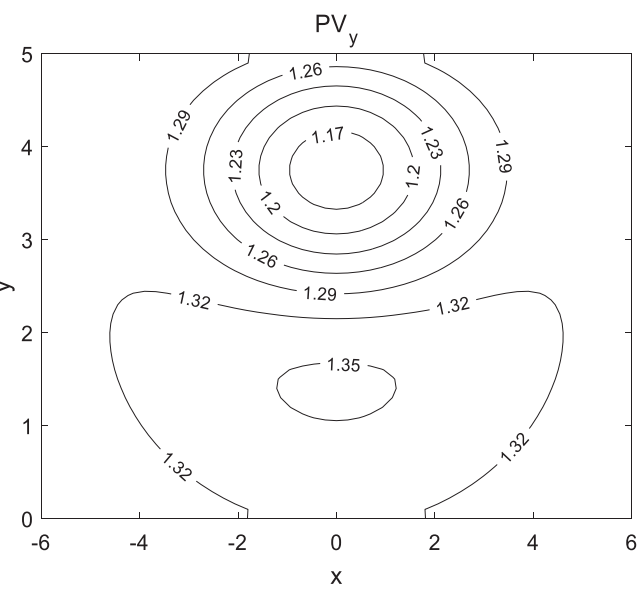

(b)

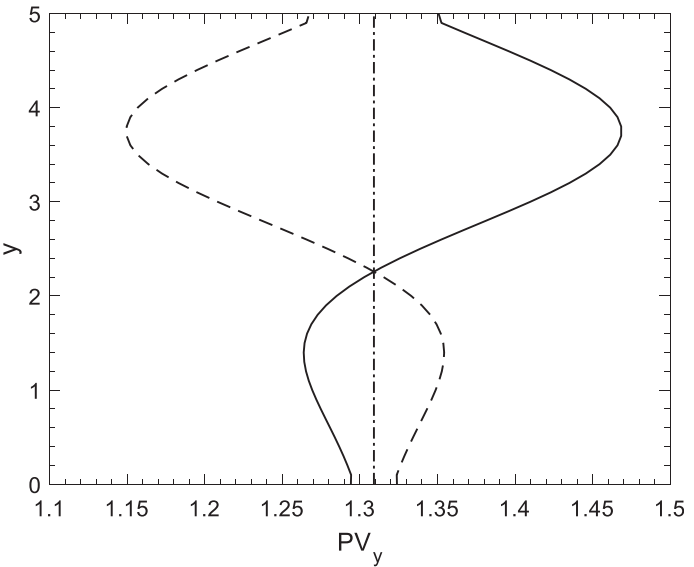

(d)

FIG. 3. (top) Horizontal distributions of (a) nondimensional background westerly wind $U(x, y)$ and (b) the associated meridional PV gradient $\mathrm{PV}_{y}=\beta-U_{y y}$ for $U=u_{0}+\Delta u e^{-\gamma\left(y-y_{0}\right)^{2}-\tilde{\gamma}\left(x-x_{0}\right)^{2}}$ with $u_{0}=0.7, \Delta u=-0.2, \gamma=0.3$, $y_{0}=3.75, \tilde{\gamma}=0.1$, and $x_{0}=0$. (bottom) Meridional profiles of (c) $U(x, y)$ and (d) $\operatorname{PV}_{y}(x, y)$ at $x=0$, where the dashed (solid) line represents the case of $\Delta u=-0.2(\Delta u=0.2)$ and $\Delta u=0$ is marked by the dot-dashed line.

the length scale of the synoptic-scale eddies must be small to allow the cascade of energy to larger scales. But in our nonlinear theory as an extension of the linear wave theory, such a condition is unnecessary because the extended NMI model considers blocking as a nonlinear initial value problem. Instead, only $-\nabla \cdot\left(\mathbf{v}_{1}^{\prime} q_{1}^{\prime}\right)_{P}$ is needed to match the PV anomaly of blocking during its initiation stage to allow the upscale energy transfer of preexisting synoptic-scale eddies to the incipient blocking. Because the linear theory cannot reflect the temporal evolution of atmospheric blocking, it is useful to apply our extended NMI model to examine how the magnitude of $\mathrm{PV}_{y}^{D}$ or $\mathrm{PV}_{y}^{T}$ influence the temporal evolution of atmospheric blocking in the barotropic and baroclinic atmospheres.

In this paper, we fix the zonal wavelength of the initial blocking to examine the likely effect of $\mathrm{PV}_{y}$ and its $\mathrm{PV}_{y}^{D}$ or $\mathrm{PV}_{y}^{T}$ on the evolution of eddy-driven blocking. In the following calculation, we further classify the BWW into two cases: barotropic $U_{B}(x, y)$ and baroclinic $U_{T}(z)$ flows to examine their respective impact on eddy-driven blocking.

\section{a. Temporal evolution of barotropic blocking}

Here, we consider a barotropic case $\left[\phi(z)=1, Z_{b}=0\right.$, $U_{T}(z)=0$ and $\left.F=0\right]$ as we have noted above. We also assume $U=U_{B}(x, y)=u_{0}+\Delta u e^{-\left[\gamma\left(y-y_{0}\right)^{2}+\tilde{\gamma}\left(x-x_{0}\right)^{2}\right]}$ with $\gamma>0$ and $\tilde{\gamma}>0$, where $u_{0}$ is a constant that represents the uniform part of the barotropic BWW, with $\Delta u$ being its horizontal shear, and $x_{0}$ and $y_{0}$ are the zonal and meridional positions of the maximum shear of the BWW. For $u_{0}=0.7, \gamma=0.3, y_{0}=3.75, \tilde{\gamma}=0.1$, and $x_{0}=0$, we show the horizontal distributions of $U(x, y)$ and associated 

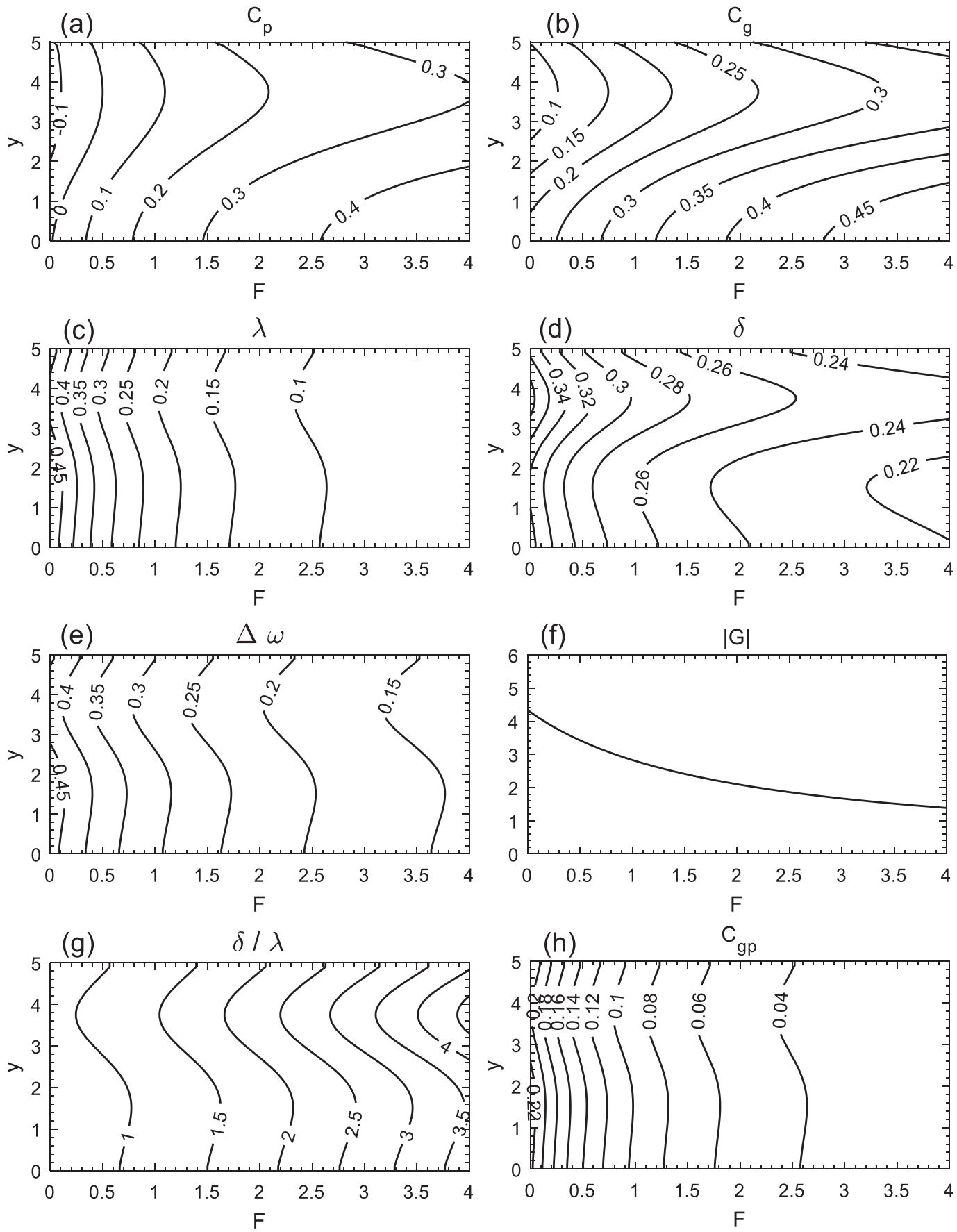

FIG. 4. Variations of (a) linear phase speed $C_{p}$, (b) group velocity $C_{g}$, (c) linear dispersion term $\lambda$, (d) nonlinearity strength $\delta$, (e) the eddy forcing frequency $\Delta \omega$, (f) eddy forcing coefficient $|G|$, (g) $\delta / \lambda$, and (h) $C_{g p}=C_{g}-C_{p}$ with the latitude $y$ and the parameter $F$ for $\mathrm{PV}_{y}=\beta-U_{y y}$ and a slowly varying background zonal wind $U=u_{0}+\Delta u e^{-\gamma\left(y-y_{0}\right)^{2}}$ with $u_{0}=0.7, \Delta u=-0.2, \gamma=0.3$, and $y_{0}=3.75$.

$\mathrm{PV}_{y}=\beta+\mathrm{PV}_{y}^{D}$ with $\mathrm{PV}_{y}^{D}=V_{x y}-U_{y y}$ in Figs. $3 \mathrm{a}$ and $3 \mathrm{~b}$ for $\Delta u=-0.2$. This figure shows that there are smaller barotropic $\mathrm{BWW}$ and $\mathrm{PV}_{y}$ in higher latitudes for $\Delta u=-0.2$. In contrast, larger barotropic BWW and $\mathrm{PV}_{y}$ are seen in higher latitudes for $\Delta u=0.2$ (not shown). Correspondingly, the meridional profiles of $U(x, y)$ and associated $\mathrm{PV}_{y}$ at $x=0$ are shown in Figs. 3c and $3 \mathrm{~d}$ for $\Delta u=-0.2$ (dashed line), $\Delta u=0$ (dot-dashed line), and $\Delta u=0.2$ (solid line). It is also found that in the high latitudes, $\mathrm{PV}_{y}$ decreases from 1.47 to 1.15 (Fig. 3d) 


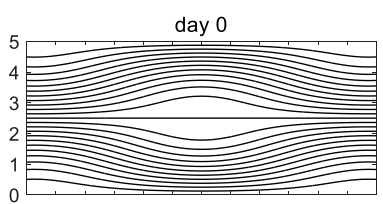

day 6
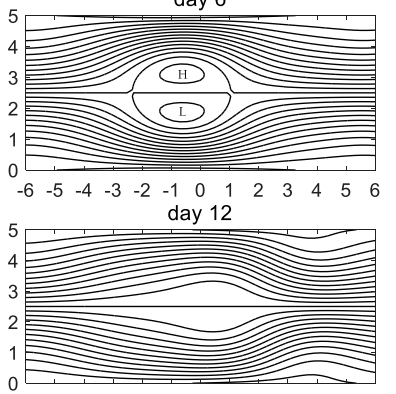

day 18

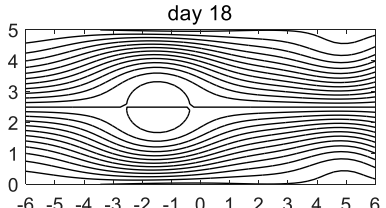

day 3

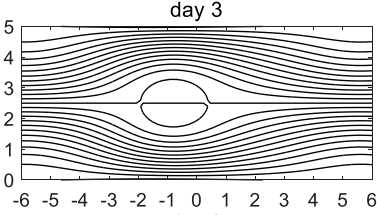

day 9

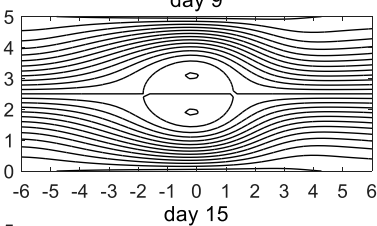

day 15

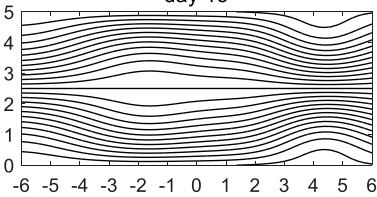

day 21

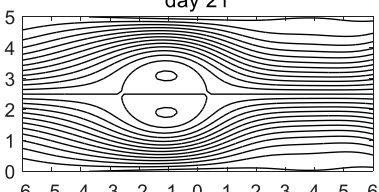

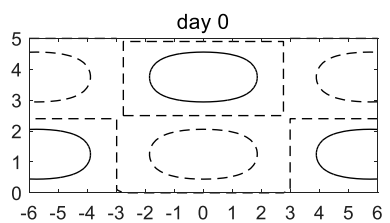

day 6

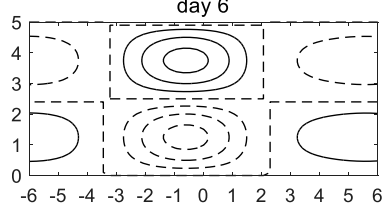

day 12

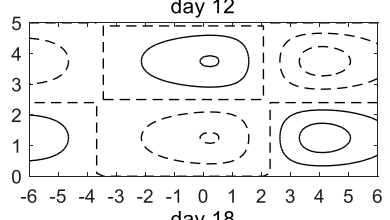

day 18

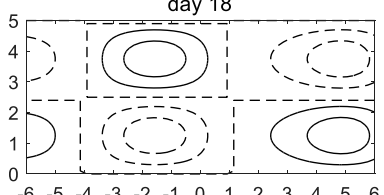

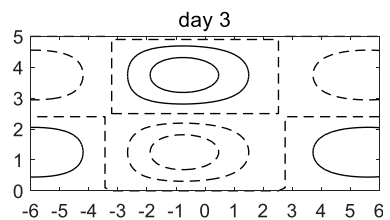

day 9

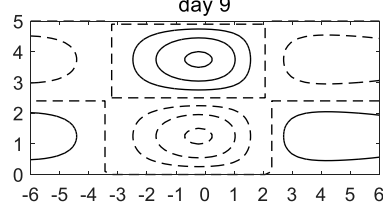

day 15

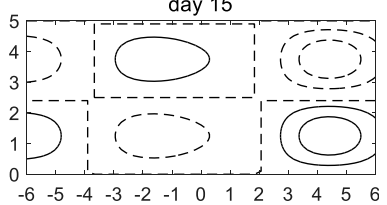

day 21

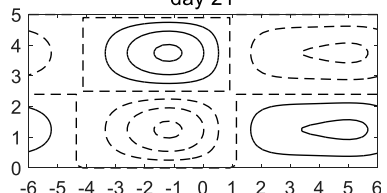

(a) $\psi_{P}$

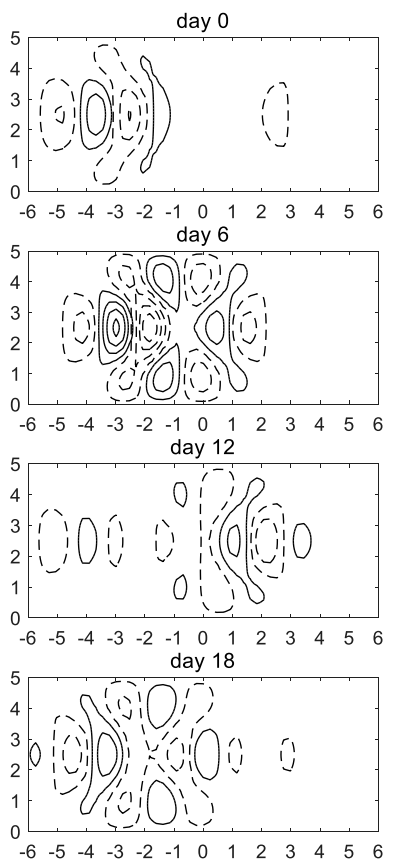

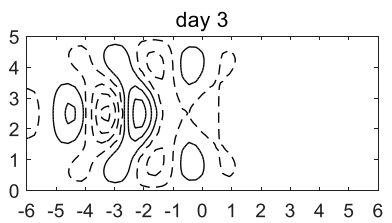

day 9

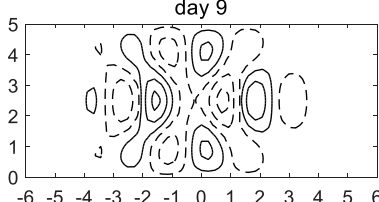

day 15
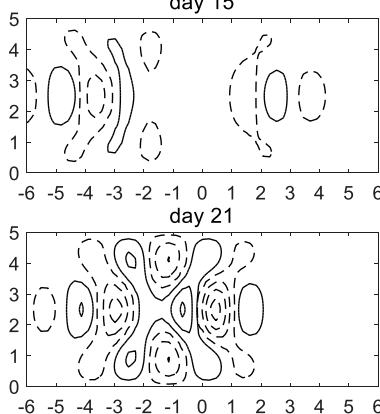

(b) $\psi_{B}$

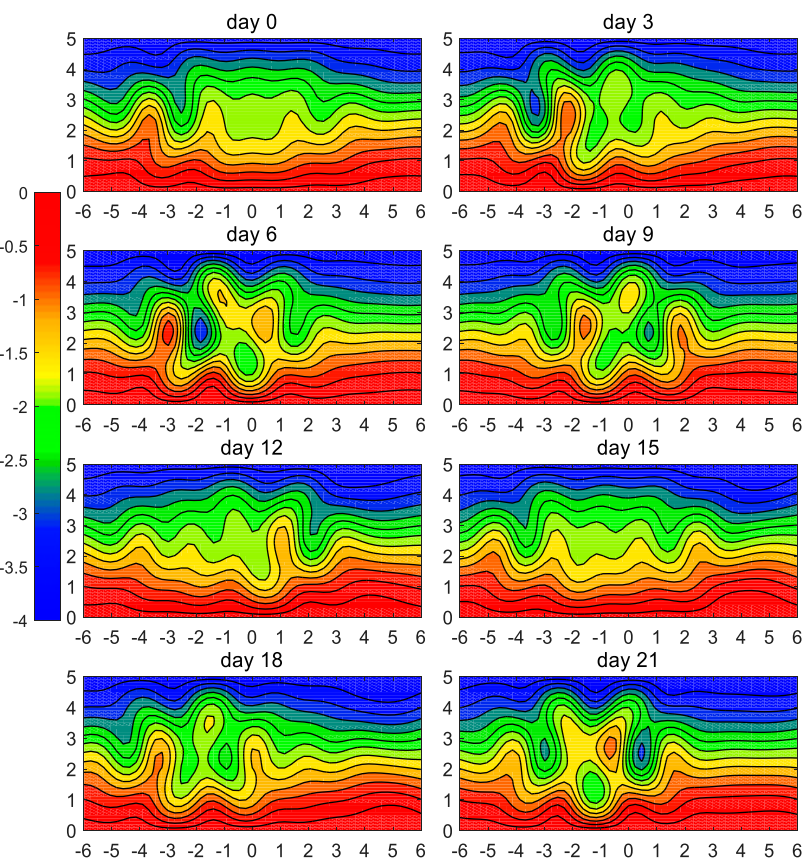

(d) $\psi_{T}$

(c) $\psi_{e}^{\prime}$

FIG. 5. Instantaneous fields of (a) planetary-scale streamfunction $\psi_{P}$ [contour interval $(\mathrm{CI})=0.15$ ], (b) blocking wavy anomaly $\psi_{B}(\mathrm{CI}=$ $0.2)$, (c) synoptic-scale eddy streamfunction $\psi_{e}^{\prime}(\mathrm{CI}=0.3)$, and $(\mathrm{d})$ total streamfunction $\psi_{T}(\mathrm{CI}=0.3)$ of eddy-driven barotropic blocking $\left[\phi(z)=1, Z_{b}=0\right.$ and $\left.F=0\right]$ from an incipient block with $\mathrm{PV}_{y}=\beta$ in an uniform background westerly wind $U=u_{0}$ with $u_{0}=0.7$ for the parameters shown in Table 1. The dashed line represents the cyclonic anomaly. The red (green and blue) shading denotes the high (low) pressure region, which is the same as below. 

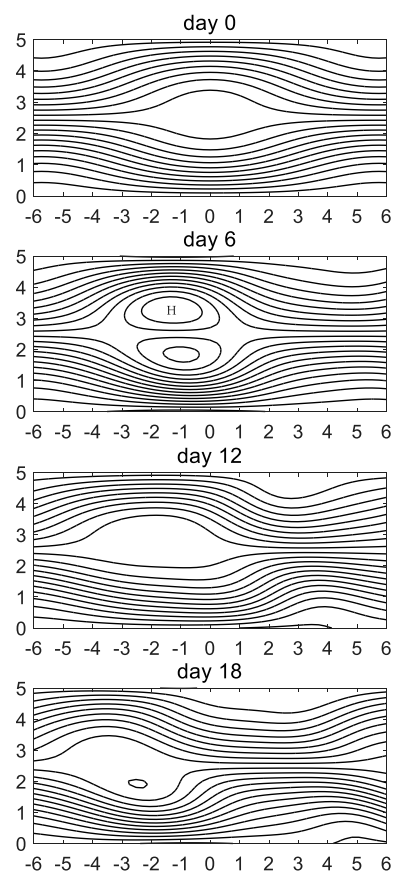

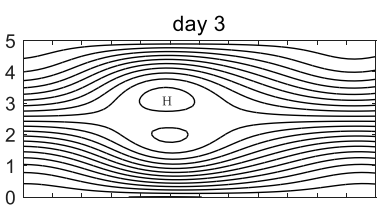

day 9

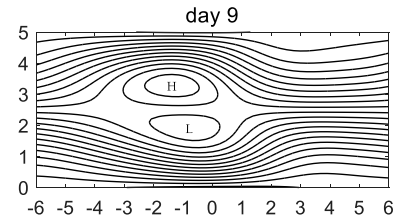

day 15

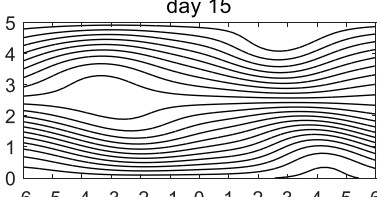

day 21

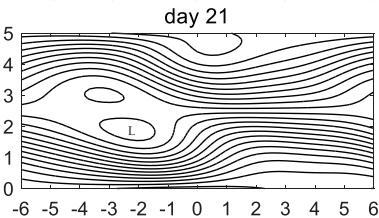

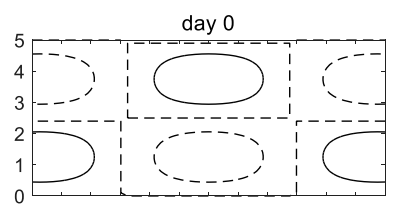

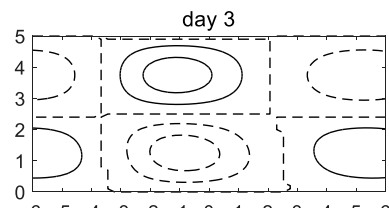

day 6

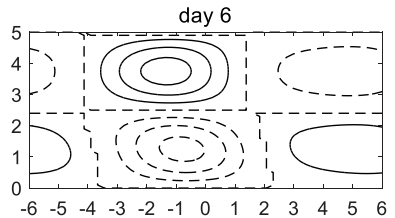

day 12
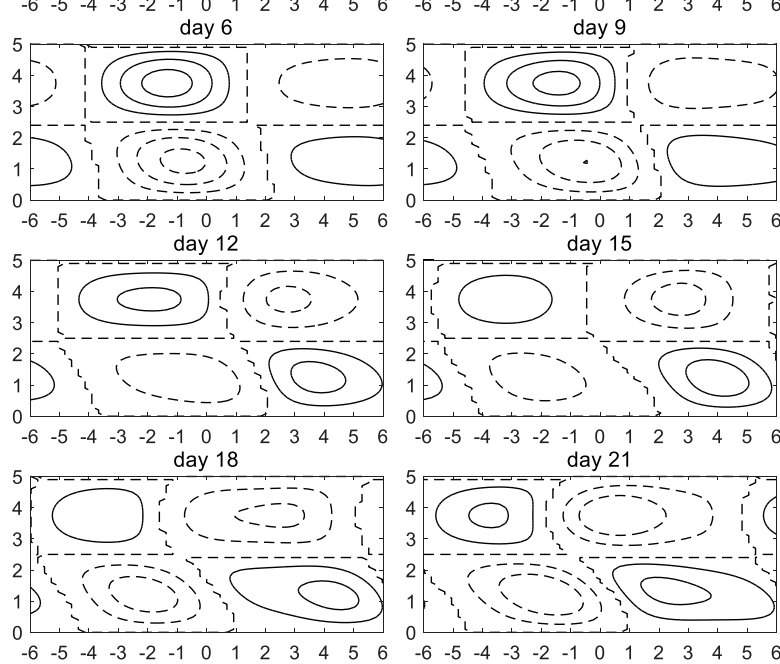

(a) $\psi_{P}$
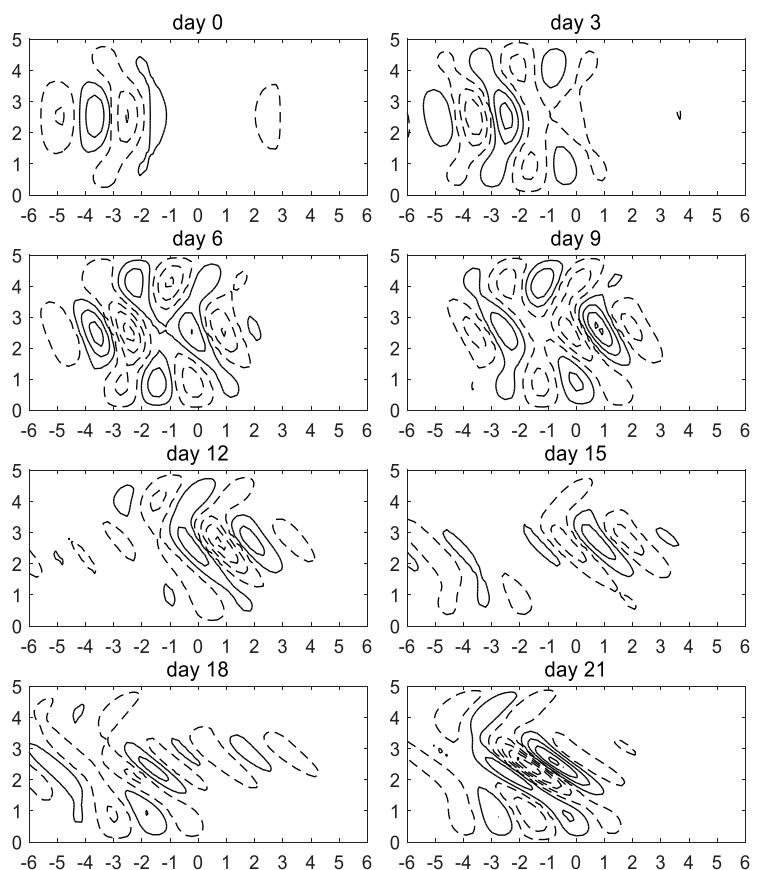

(c) $\psi_{e}^{\prime}$ (b) $\psi_{B}$
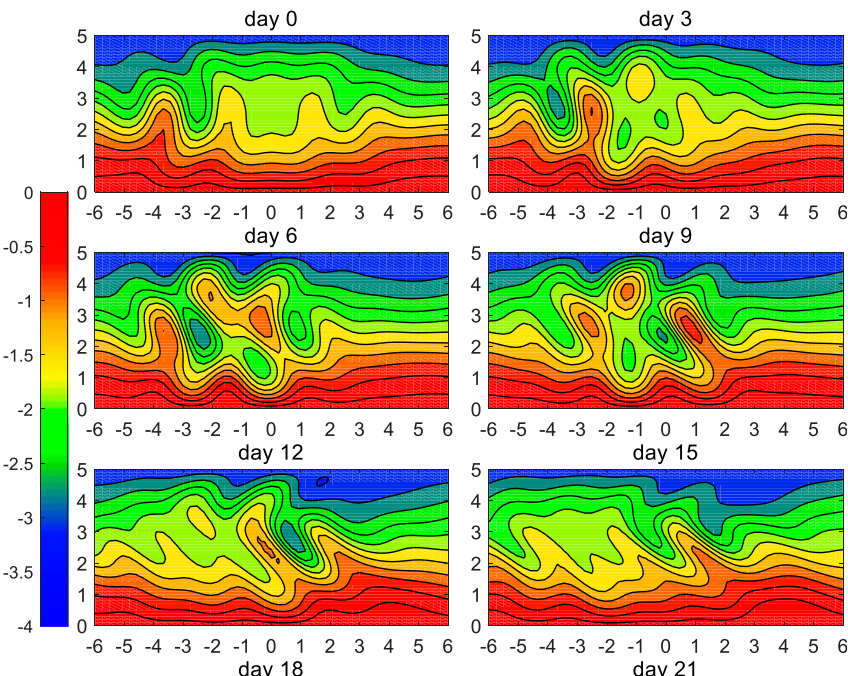

day 15
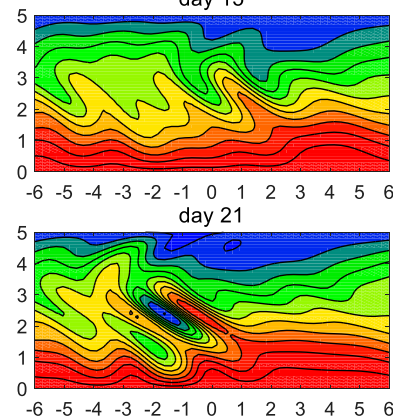

(d) $\psi_{T}$

FIG. 6. As in Fig. 5, but for $\mathrm{PV}_{y}=\beta-U_{y y}$ in a weak slowly varying background westerly wind being weaker in the higher latitudes for $U=u_{0}+\Delta u e^{-\gamma\left(y-y_{0}\right)^{2}}$ with $u_{0}=0.7, \Delta u=-0.2, \gamma=0.3$, and $y_{0}=3.75$. 
when the barotropic BWW strength decreases from 0.9 to 0.5 along $y=3.75$ (Fig. 3c). In this section, we examine how such barotropic BWW and $\mathrm{PV}_{y}$ distributions affect the evolution of eddy-driven blocking. Below we only present the results for $\gamma=0.3$ and $\tilde{\gamma}=0$ because the value of $\tilde{\gamma}$ does not significantly change our calculation results (not shown).

Figure 4 shows the variations of $C_{p}, C_{g}, \lambda, \delta, \Delta \omega,|G|$, $\delta / \lambda$, and $C_{g p}$ with the latitude and the value of $F$ for $U(y)=u_{0}+\Delta u e^{-\gamma\left(y-y_{0}\right)^{2}}$ with $u_{0}=0.7, \gamma=0.3, y_{0}=3.75$, and $\Delta u=-0.2$. Clearly, when the barotropic BWW is small in higher latitudes, $\delta / \lambda$ is large (Fig. $4 \mathrm{~g}$ ) and $C_{g p}$ and $\Delta \omega$ are small (Figs. $4 \mathrm{~h}, \mathrm{e}$ ). Thus, a weaker $\mathrm{PV}_{y}$ in higher latitudes related to a smaller BWW as a weaker dispersion or a stronger nonlinearity media may provide a favorable environment for the longer lifetime of blocking in a certain range of $u_{0}$. We also examine the sensitivity of the blocking evolution to the value of $u_{0}$.

Using the same parameters as in Table 1, we show instantaneous fields of planetary-scale streamfunction $\psi_{P}$, blocking wavy anomaly $\psi_{B}$, synoptic-scale eddy streamfunction $\psi_{e}^{\prime}\left[\psi_{e}^{\prime}=\phi(z)\left(\psi_{1}^{\prime}+\psi_{2}^{\prime}\right)\right]$ and total streamfunction $\psi_{T}\left(\psi_{T}=\psi_{P}+\psi_{e}^{\prime}\right)$ of eddy-driven barotropic blocking $[\phi(z)=1]$ during its life cycle in Fig. 5 for a uniform BWW with $U=u_{0}=0.7$. It is noted that initial synoptic-scale eddies $\psi_{e}^{\prime}\left(\psi_{e}^{\prime}=\psi_{1}^{\prime}+\psi_{2}^{\prime}\right)$ (Fig. 5c for day 0$)$ are located in the upstream side of an incipient block located at $x=0$ (Fig. 5a for day 0 ). Through the forcing of $-\nabla \cdot\left(\mathbf{v}_{1}^{\prime} q_{1}^{\prime}\right)_{P}$ caused by the preexisting eddies $\psi_{1}^{\prime}$, the prespecified initial blocking can be reinforced into a typical dipole blocking (Figs. 5a and 5b for day 6). This blocking has a lifetime less than 15 days because it disappears completely at day 15 . A new blocking reappears due to the reappearance of an anticyclonic-overcyclonic dipole forcing of $-\nabla \cdot\left(\mathbf{v}_{1}^{\prime} q_{1}^{\prime}\right)_{P}$ after day 15 (Luo et al. 2014). The synoptic-scale eddies $\psi_{e}^{\prime}$ are intensified, strained and split into two branches (Fig. 5c) around the blocking region due to the feedback of intensified dipole blocking (Fig. 5b) on the preexisting synoptic eddies $\psi_{1}^{\prime}$ because deformed eddies $\psi_{2}^{\prime}$ change with the variation of the envelope amplitude $B$ of blocking.

When the blocking disappears or when $B=0, \psi_{2}^{\prime}$ vanishes, which corresponds to the absence of eddy straining or CWB. Thus, the eddy straining and CWB are a result of the blocking intensification, which tend to occur together with the blocking establishment. In this case, it is not difficult to conclude that the preexisting synoptic eddies rather than the eddy straining play a key role in the blocking formation, maintenance, and decay, which is different from the eddy straining mechanism of Shutts (1983). In the total streamfunction field $\psi_{T}$ (Fig. 5d), the establishment of eddy-driven blocking is characterized by a large meandering of westerly jet streams, which is composed of several isolated synoptic-scale cyclonic and anticyclonic vortices within the blocking region. A large meandering of the westerly jet streams also reflects the presence of CWB or eddy straining related to the northward (southward) displacement of intensified synoptic-scale ridges (troughs) in the blocking region and its upstream side.

Here, we further examine how reduced barotropic BWW and $\mathrm{PV}_{y}$ in higher latitudes influence the evolution of eddy-driven barotropic blocking. For $U(y)=$ $u_{0}+\Delta u e^{-\gamma\left(y-y_{0}\right)^{2}}$ with $u_{0}=0.7, \Delta u=-0.2, \gamma=0.3$, and $y_{0}=3.75$, we show the instantaneous $\psi_{P}, \psi_{B}, \psi_{e}^{\prime}$, and $\psi_{T}$ fields of eddy-driven barotropic blocking $[\phi(z)=1]$ in Fig. 6 during its evolution process. It is interesting to note that the barotropic blocking shows a northwestsoutheast (NW-SE)-oriented dipole due to the presence of small barotropic BWW and $\mathrm{PV}_{y}$ in higher latitudes, which undergoes an enhanced retrogression and is further intensified (Figs. 6a,b) relative to that in a uniform BWW (Figs. 5a,b). The lifetime of this barotropic blocking is slightly lengthened as well. In Fig. 6c, we can see a significant horizontal straining of synoptic-scale eddies due to the meridional shear of the barotropic BWW with a smaller strength in higher latitudes than in lower latitudes (Fig. 3c) and due to the feedback of intensified NW-SE-oriented blocking dipole (Fig. 6b). The strained synoptic-scale eddies move eastward more rapidly over the south side of the blocking region than over its north side (Fig. 6c) so that the spatial pattern of the westerly jet stream meandering during the blocking life cycle (Fig. 6d) is slightly different from that in a uniform BWW (Fig. 5d).

To see the impact of the barotropic BWW and $\mathrm{PV}_{y}$ on the lifetime and local persistence of eddy-driven dipole blocking, the maximum amplitude $\psi_{\text {Max }}$ of the anticyclonic anomaly of blocking wavy part $\psi_{B}$ at $y=3.75$ for each day is defined as the daily strength of dipole blocking to characterize the lifetime of blocking. It is also useful to consider the value of the domain-averaged blocking wavy anomaly streamfunction $\psi_{A}$ over $-1.5 \leq$ $x \leq-0.5$ and $3.5 \leq y \leq 4$ at every day during the blocking life cycle as an daily index reflecting the local persistence of blocking because the eddy-driven blocking often moves upstream in a weak BWW. We show the time-longitude evolution of the blocking wavy anomaly $\psi_{B}$ at $y=3.75$ as reflecting the propagation or movement of blocking wave packet and the time series of daily $\psi_{\text {Max }}$ and $\psi_{A}$ during the blocking life cycle in Fig. 7 for $u_{0}=0.7$ and $u_{0}=0.5$ of $U=u_{0}+\Delta u e^{-\gamma\left(y-y_{0}\right)^{2}}, \gamma=0.3$, and $y_{0}=3.75$ with three cases: $\Delta u=-0.2, \Delta u=0$, and $\Delta u=0.2$. 

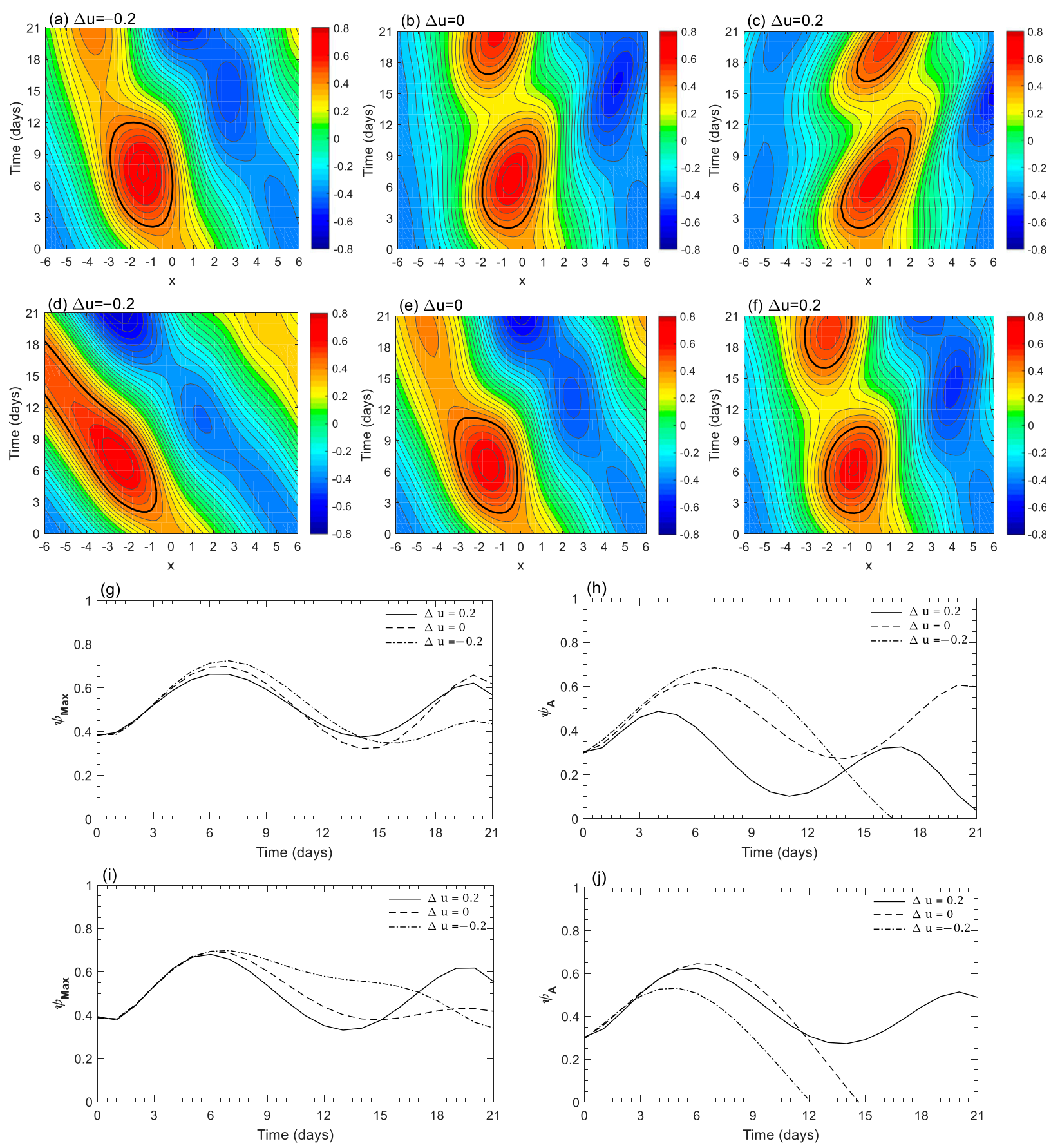

FIG. 7. (a)-(f) Time-longitude evolution of the blocking wavy anomaly $\psi_{B}(\mathrm{CI}=0.05$ and 0.45 is marked by the thick black line) at $y=3.75$ of barotropic blocking $\left[\phi(z)=1, Z_{b}=0\right.$, and $\left.F=0\right]$ for (a),(d) weak $(\Delta u=-0.2)$ and (c),(f) strong $(\Delta u=0.2)$ barotropic background westerly winds in higher latitudes and (b),(e) uniform barotropic background westerly wind $(\Delta u=0)$ for $U=u_{0}+\Delta u e^{-\gamma\left(y-y_{0}\right)^{2}}$ and $\mathrm{PV}_{y}=\beta-U_{y y}$ with (a)-(c) $u_{0}=0.7$ and (d)-(f) $u_{0}=0.5, \gamma=0.3$, and $y_{0}=3.75$. (g)-(j) Temporal variations of the (g),(i) maximum daily amplitude $\psi_{\text {Max }}$ of a blocking anticyclone and (h),(j) domain-averaged daily amplitude $\psi_{A}$ over $-1.5 \leq x \leq-0.5$ and $3.5 \leq y \leq 4$ for (g),(h) $u_{0}=0.7$ and (i),(j) $u_{0}=0.5$ with $\Delta u=-0.2$ (dot-dashed line), $\Delta u=0$ (dashed line), and $\Delta u=0.2$ (solid line).

It is seen that the lifetime, strength, and movement of eddy-driven barotropic blocking depend on the strength of the barotropic BWW and its spatial distribution (Figs. 7a-f). Figures 7a-c (Figs. 7d-f) show the case of $u_{0}=0.7\left(u_{0}=0.5\right)$. In general, the lifetime of the barotropic blocking is less than 15 days (thick black lines in Figs. 7a-c,e,f), unless the barotropic BWW is particularly small in higher latitudes (Fig. 7d). When the BWW is 
stronger in higher latitudes, the barotropic blocking moves eastward (Fig. 7c) due to large high-latitude $\mathrm{PV}_{y}$. In contrast, the eastward movement of blocking (Fig. 7b) is suppressed and even its slow retrogression can be seen (Fig. 7a), when $U$ or $\mathrm{PV}_{y}$ is small in higher latitudes. Specifically, when the uniform part of $U$ (e.g., $\left.u_{0}=0.5\right)$ is smaller (Fig. 7d), the barotropic blocking shows more notable retrogression, slower decay, and has longer lifetime, while its life period is slightly short for $\Delta u=0$ (Fig. 7e) or $\Delta u=0.2$ (Fig. 7f) compared to that of $u_{0}=0.7$ for $\Delta u=0$ (Fig. 7b) or $\Delta u=0.2$ (Fig. 7c). The movement of eddy-driven blocking as a wave packet propagation can be explained by using the nonlinear phase speed of $C_{\mathrm{NP}}=U-\mathrm{PV}_{y} /\left(k^{2}+m^{2}+F\right)-\delta_{N} M_{0}^{2} /\left(2 k \mathrm{PV}_{y}\right)$, where $M_{0}=\psi_{\text {Max }} /\left(2 \sqrt{2 / L_{y}}\right)$ is the amplitude of instantaneous blocking (Luo et al. 2019).

As can be seen in Figs. $7 \mathrm{~g}-\mathrm{j}$, while the lifetime and strength of eddy-driven barotropic blocking is slightly influenced by the strength of $U$ or $\mathrm{PV}_{y}$ in higher latitudes (Fig. 7g), its local persistence seems to depend more strongly on the magnitude and meridional distribution of $U$ or $\mathrm{PV}_{y}$ (Fig. 7h). When the uniform part of $U$ is particularly small, a long-lived blocking with large retrogression and slow decay is easily seen (Fig. 7i). For this case, the difference of the local persistence of blocking becomes less distinct among $\Delta u=-0.2, \Delta u=$ 0 , and $\Delta u=0.2$ (Fig. $7 \mathrm{j}$ ), in which the local persistence of blocking is shortest for $\Delta u=-0.2$. The above results are obtained based on a pure barotropic case $\left(Z_{b}=0\right.$, $F=0$, and $\mathrm{PV}_{y}^{T}=0$ ).

It needs to be pointed out that the barotropic blocking becomes a baroclinic case even for $Z_{b}=0$ and $F=0$, if there are $\mathrm{PV}_{y}=\beta+\mathrm{PV}_{y}^{T}$ and $\mathrm{PV}_{y}^{T}=F_{r} U_{z}-F_{r} U_{z z} \neq 0$ for $\mathrm{PV}_{y}^{D}=0$. This kind of blocking is still referred to as a baroclinic blocking because it shows a vertical variation even for $\mathrm{PV}_{y}^{T}=0$, when $Z_{b} \neq 0$ and $F \neq 0$. These problems are further examined in the next subsection.

\section{b. Effect of barotropic background westerly wind on the evolution of baroclinic blocking in the baroclinic atmosphere with stratification}

In a baroclinic atmosphere with $Z_{b}=0.2$ and $F=0.82$ we show the $\psi_{P}, \psi_{B}, \psi_{e}^{\prime}$, and $\psi_{T}$ fields of eddy-driven baroclinic blocking at $z=0$ during its life cycle in Fig. 8 for $\Delta u=0$ and $\Delta u=-0.2$ of $U(y)=u_{0}+\Delta u e^{-\gamma\left(y-y_{0}\right)^{2}}$ with $u_{0}=0.7, \gamma=0.3$, and $y_{0}=3.75$. It is found that for $\Delta u=0$ (a uniform barotropic BBW) the baroclinic blocking shows an antisymmetric dipole, long lifetime and notable eastward movement especially during the blocking decaying phase (Figs. 8a,b), whereas it shows a NW-SE orientation and a reduced eastward movement for $\Delta u=-0.2$ (Figs. 8c,d). A large increase in the local persistence of blocking is also seen in this case. For the same conditions as in Figs. 8c and 8d, the eddy-driven blocking can have large amplitude in the midtroposphere $(z=0.5)$ as shown in Fig. 9a. We note that the blocking amplitude increases with height and then decreases with height above the midtroposphere, as seen from a comparison between Figs. 9a and 8c. The evolving synoptic-scale eddies $\psi_{e}^{\prime}$ also show a similar behavior (Fig. 9c). Thus, a distinctly large meandering of westerly jet streams mainly appears in the midtroposphere (Fig. 9d) where the blocking amplitude and eddy straining or CWB are strong.

Figure 10 shows the result of $\Delta u=0.2$ in the midtroposphere for the same parameters as in Fig. 9. It is seen that the eddy-driven blocking exhibits a northeastsouthwest (NE-SW) orientated dipole structure and remains rather strong even after day 18 (Figs. 10a,b) because the barotropic $\mathrm{BWW}$ or $\mathrm{PV}_{y}$ is stronger in higher latitudes than in lower latitudes (solid lines in Figs. $3 c$ and $3 d$ ). In addition, because the synoptic-scale eddies move eastward more rapidly in higher latitudes than in lower latitudes (Fig. 10c), the spatial pattern of the meandering westerly jet stream (Fig. 10d) is different from that of $\Delta u=-0.2$ as seen in Fig. $9 \mathrm{~d}$. Thus, while the local persistence of eddy-driven blocking is weak for $\Delta u=0.2$, it has long lifetime, fast eastward movement, and slow decay (Figs. 10a,b).

For $U(y)=u_{0}+\Delta u e^{-\gamma\left(y-y_{0}\right)^{2}}, \gamma=0.3, y_{0}=3.75, Z_{b}=$ 0.2 , and $F=0.82$ with three cases of $\Delta u=-0.2, \Delta u=0$, and $\Delta u=0.2$, we show the time-longitude evolution of the blocking wavy anomaly $\psi_{B}$ at $y=3.75$ and the time series of daily $\psi_{\text {Max }}$ and $\psi_{A}$ of eddy-driven baroclinic blocking during the blocking life cycle in Fig. 11 for $u_{0}=$ 0.7 and $u_{0}=0.5$. It is found that the eddy-driven baroclinic blocking can have longer lifetime in the baroclinic atmosphere with stratification $\left(Z_{b}=0.2\right.$ and $\left.F=0.82\right)$ (Figs. 11a-f) than in the pure barotropic atmosphere $\left(Z_{b}=0\right.$ and $F=0$ ) (Figs. 7a-c,e-f), even when the barotropic BWW is less weak. The evolution (movement, lifetime, strength) of blocking is also found to depend on the magnitude of $u_{0}$ or $U$ and $\mathrm{PV}_{y}^{D}$. While the life time (eastward movement) of eddy-driven baroclinic blocking is increased (intensified) with the increasing of $U$ and $\mathrm{PV}_{y}^{D}$ (Figs. 11a-c), its local persistence is weakened. We also see that for $u_{0}=0.7$, the slow decay of this baroclinic blocking is stronger for $\Delta u=0.2$ (solid line in Fig. 11g) than that for $\Delta u=-0.2$ (dot-dashed line in Fig. 11g) or $\Delta u=0$ (dashed line in Fig. 11g).

In addition, the value of the uniform part $u_{0}$ of the barotropic BWW can affect the lifetime, movement, and local persistence of eddy-driven baroclinic blocking. It is noted that $\mathrm{PV}_{y}^{D}$ is the same between $u_{0}=0.7$ and $u_{0}=$ 0.5 for each case of $\Delta u=0.2, \Delta u=-0.2$, and $\Delta u=0$. While the lifetime of blocking becomes shorter for 


$$
\Delta u=0
$$
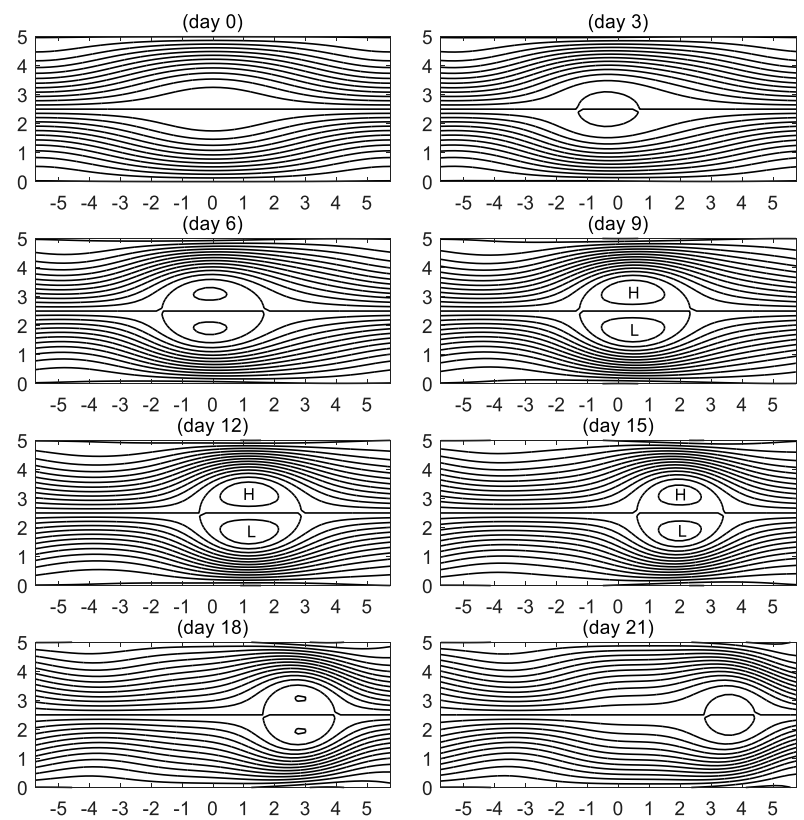

(a) $\psi_{P}$
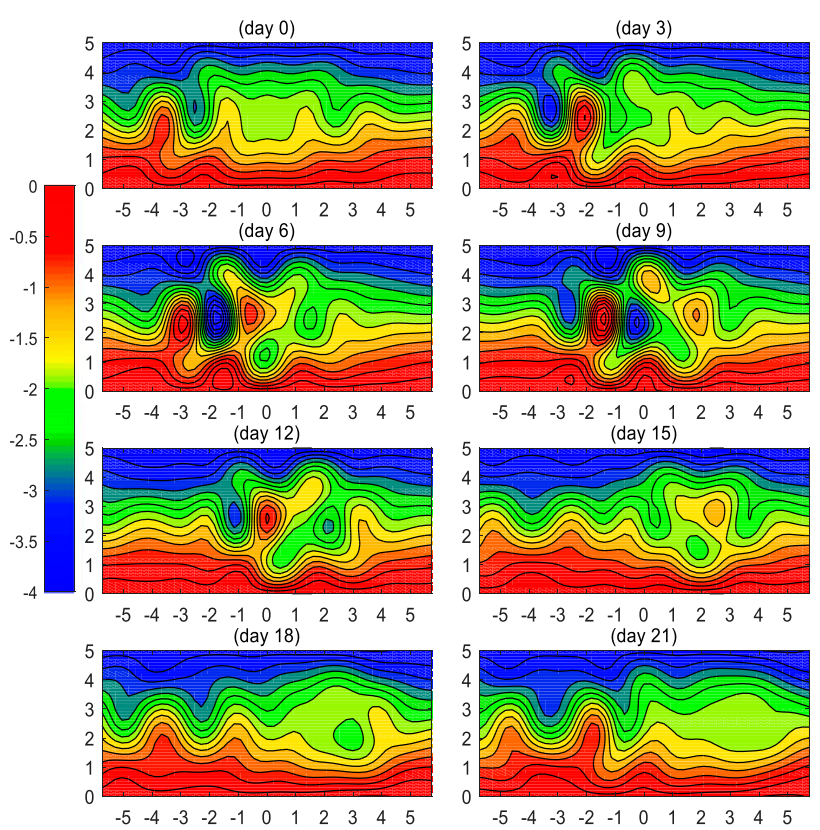

(b) $\psi_{T}$

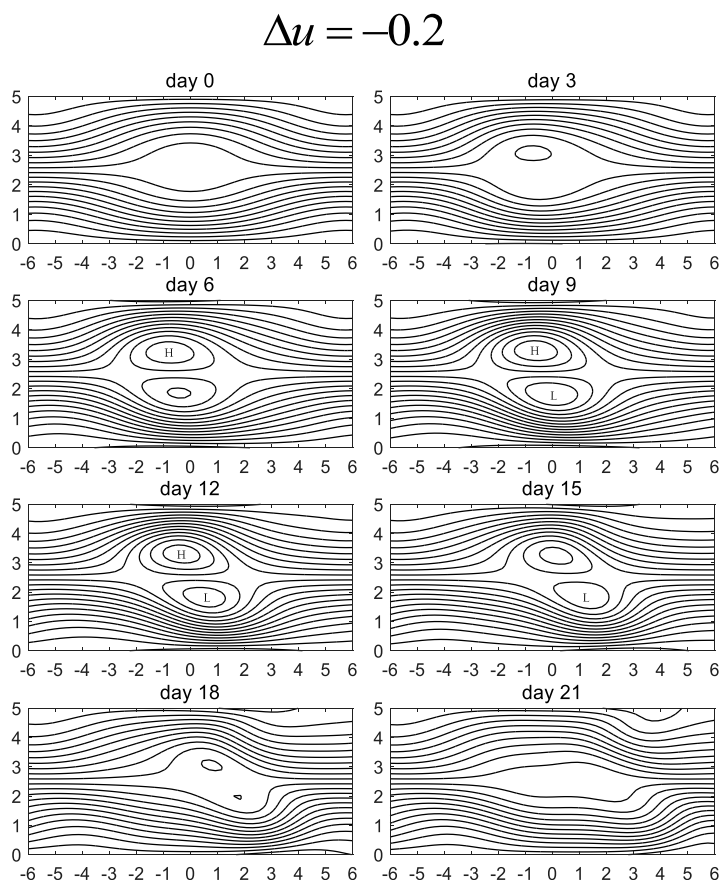

(c) $\psi_{P}$

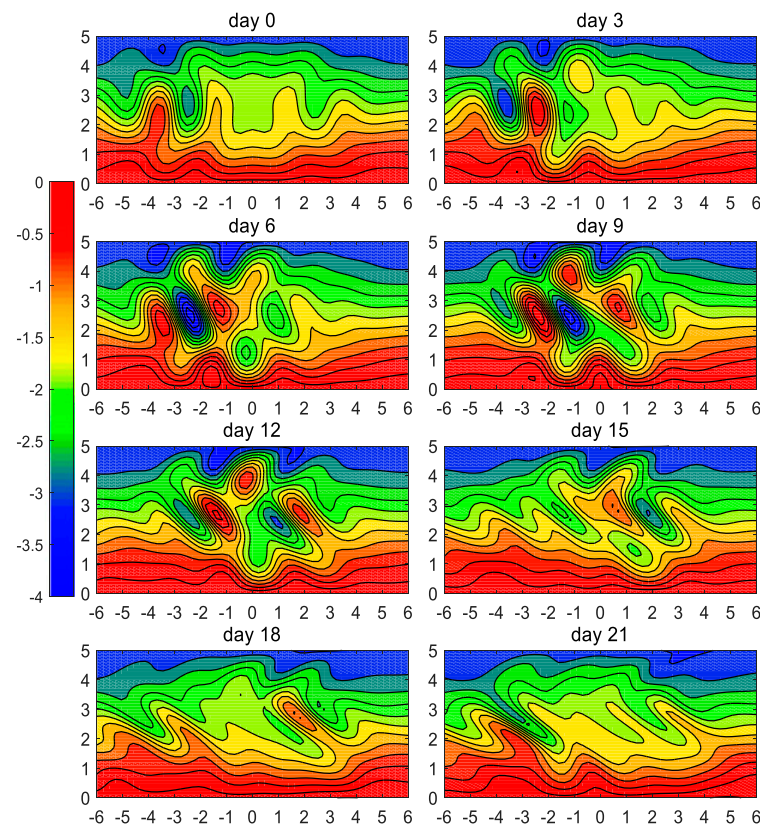

(d) $\psi_{T}$

FIG. 8. Instantaneous fields of (a),(c) planetary-scale streamfunction $\psi_{P}(\mathrm{CI}=0.15)$ and (b),(d) total streamfunction $\psi_{T}(\mathrm{CI}=0.3)$ on $z=0$ of eddy-driven baroclinic blocking $\left(Z_{b}=0.2\right.$ and $\left.F=0.82\right)$ from an incipient block for $\mathrm{PV}_{y}=\beta-U_{y y}$ and $U=u_{0}+\Delta u e^{-\gamma\left(y-y_{0}\right)^{2}}$ with $u_{0}=0.7, \gamma=0.3$, and $y_{0}=3.75$ in the (a),(b) uniform barotropic background westerly wind $(\Delta u=0)$ and (c),(d) weak high-latitude barotropic background westerly wind $(\Delta u=-0.2)$ for the parameters shown in Table 1. 

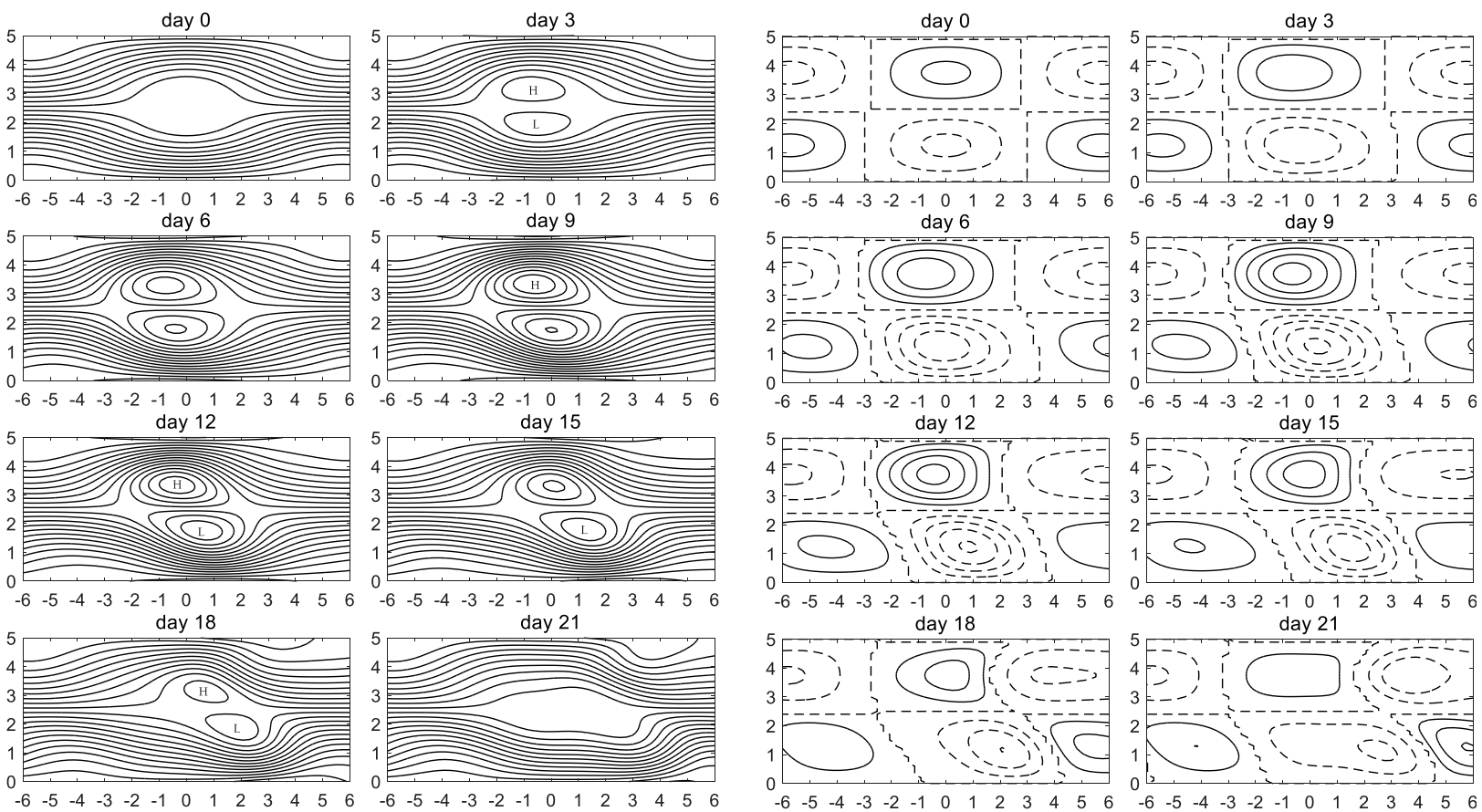

day 15
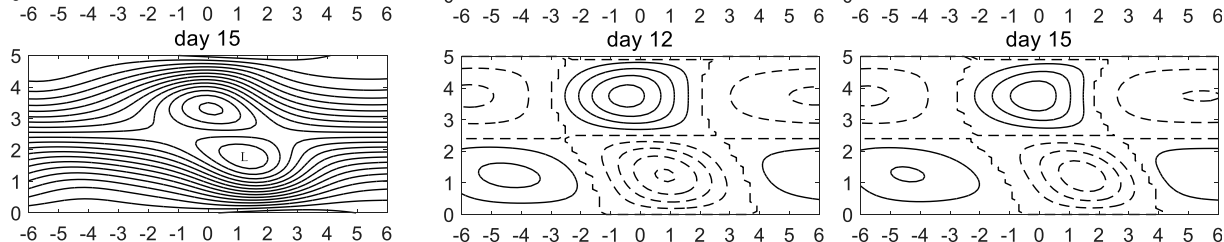

day 21

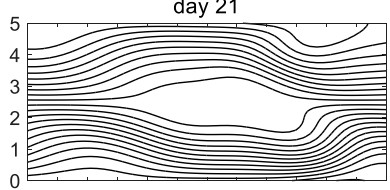

day 18
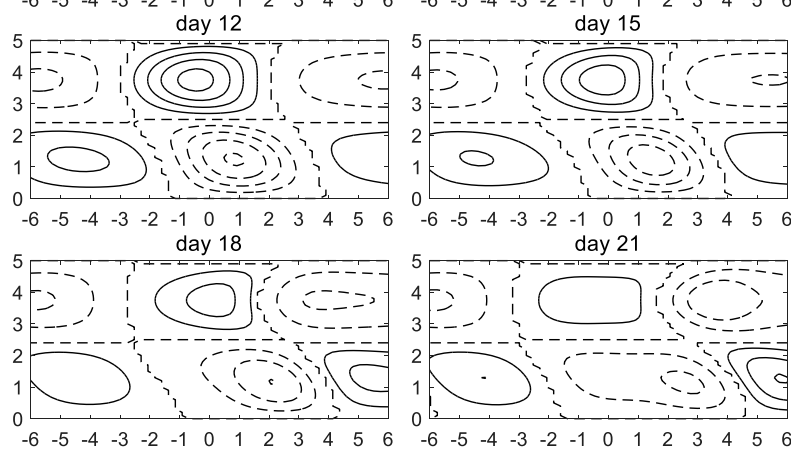

(a) $\psi_{P}$

(b) $\psi_{B}$
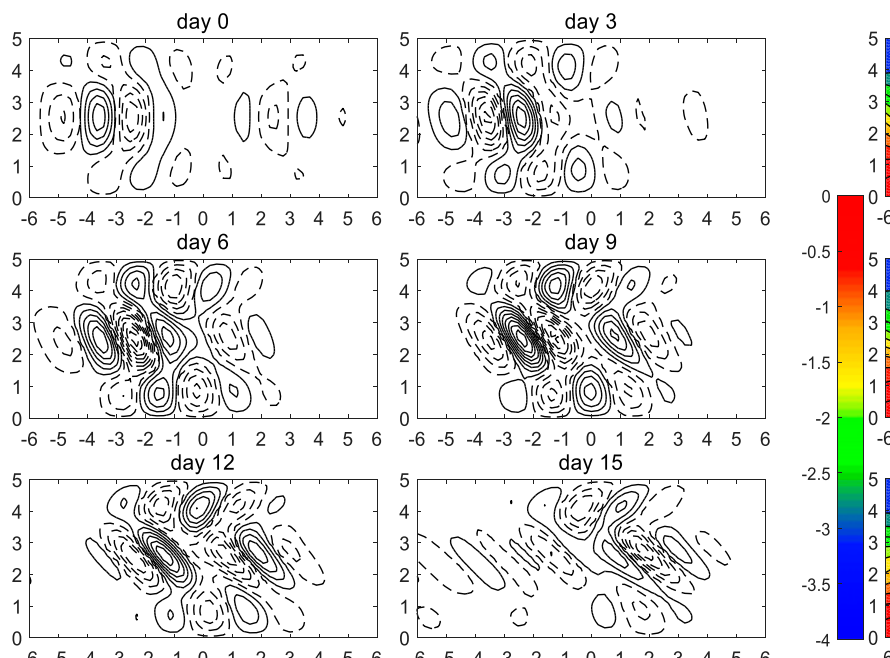

day 0

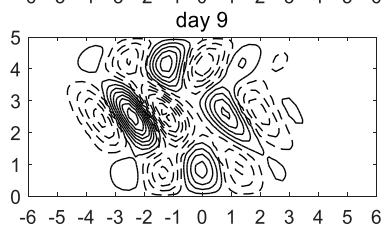
day 12

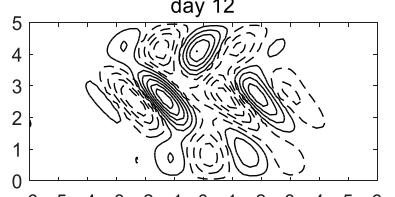

day 15
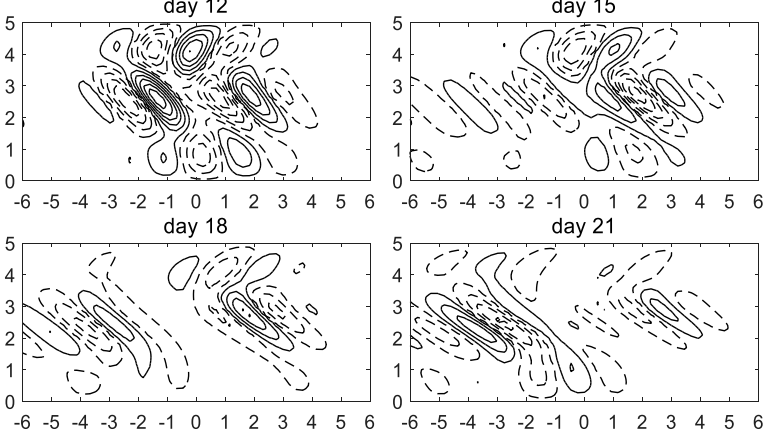

(c) $\psi_{e}^{\prime}$

(d) $\psi_{T}$

FIG. 9. Instantaneous fields of (a) planetary-scale streamfunction $\psi_{P}(\mathrm{CI}=0.15)$, (b) blocking wavy anomaly $\psi_{B}(\mathrm{CI}=0.2)$, and (c) synoptic-scale eddy streamfunction $\psi_{e}^{\prime}(\mathrm{CI}=0.3)$ and $(\mathrm{d})$ total streamfunction $\psi_{T}(\mathrm{CI}=0.3)$ in the midtroposphere $(z=0.5)$ of eddydriven baroclinic blocking $\left(Z_{b}=0.2\right.$ and $\left.F=0.82\right)$ from an incipient block with $\mathrm{PV}_{y}=\beta-U_{y y}$ in a weak high-latitude barotropic background westerly wind $U=u_{0}+\Delta u e^{-\gamma\left(y-y_{0}\right)^{2}}$ with $u_{0}=0.7, \Delta u=-0.2, \gamma=0.3$, and $y_{0}=3.75$, and for the parameters shown in Table 1 . The dashed line represents the cyclonic anomaly. 


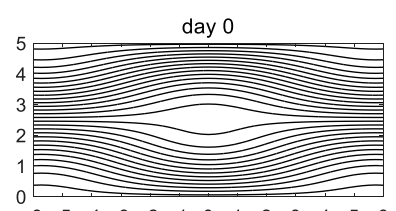

day 6
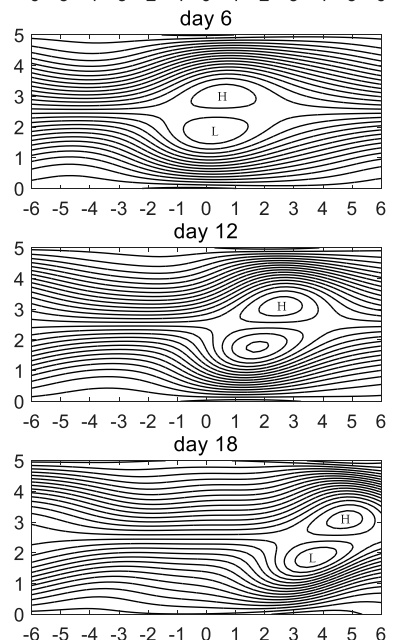

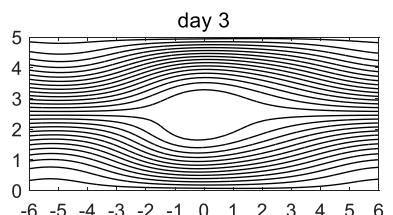

day 9
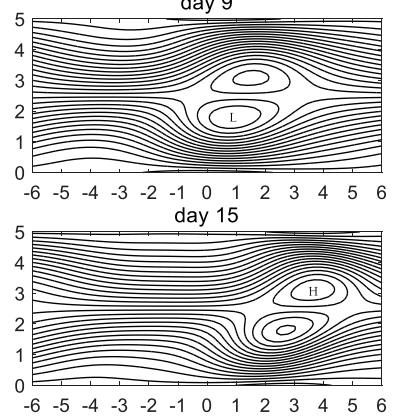

day 21

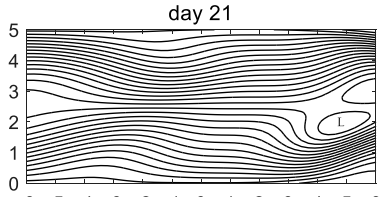

(a) $\psi_{P}$
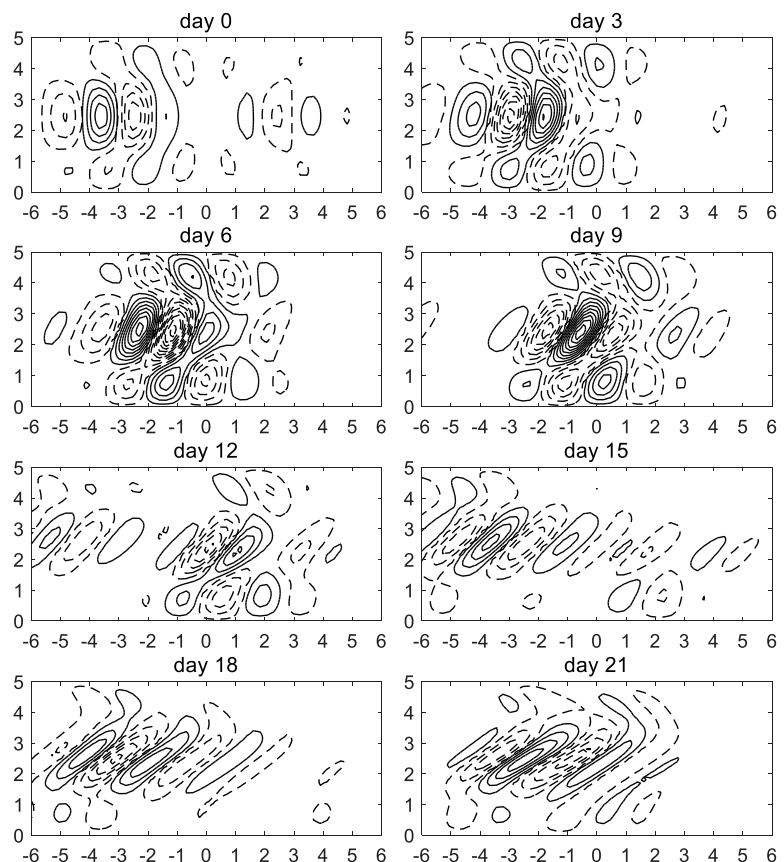

day 21

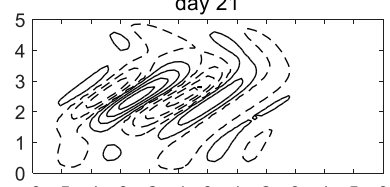

(c) $\psi_{e}^{\prime}$
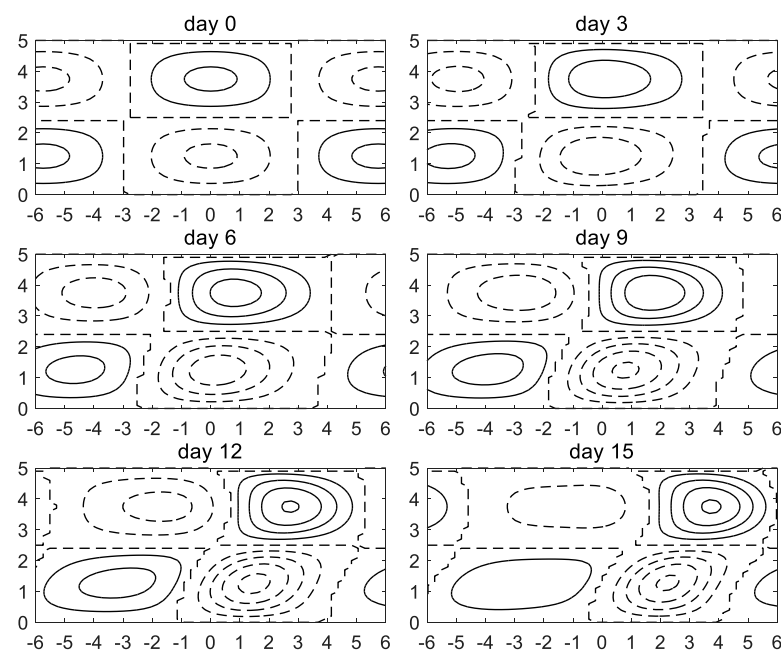

day 18
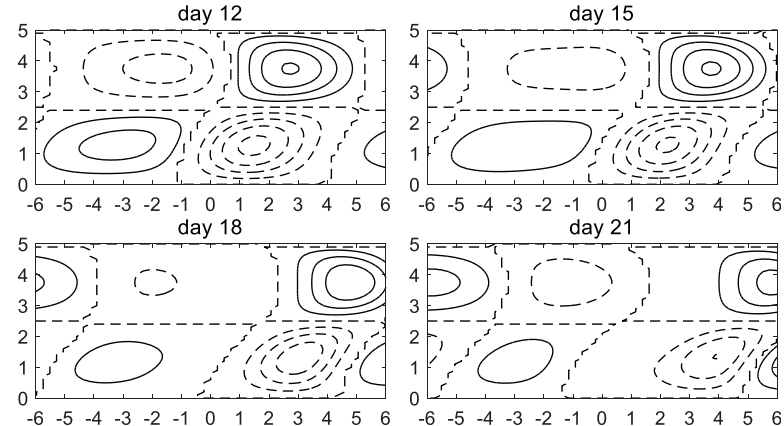

day 21

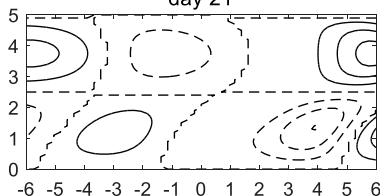

(b) $\psi_{B}$
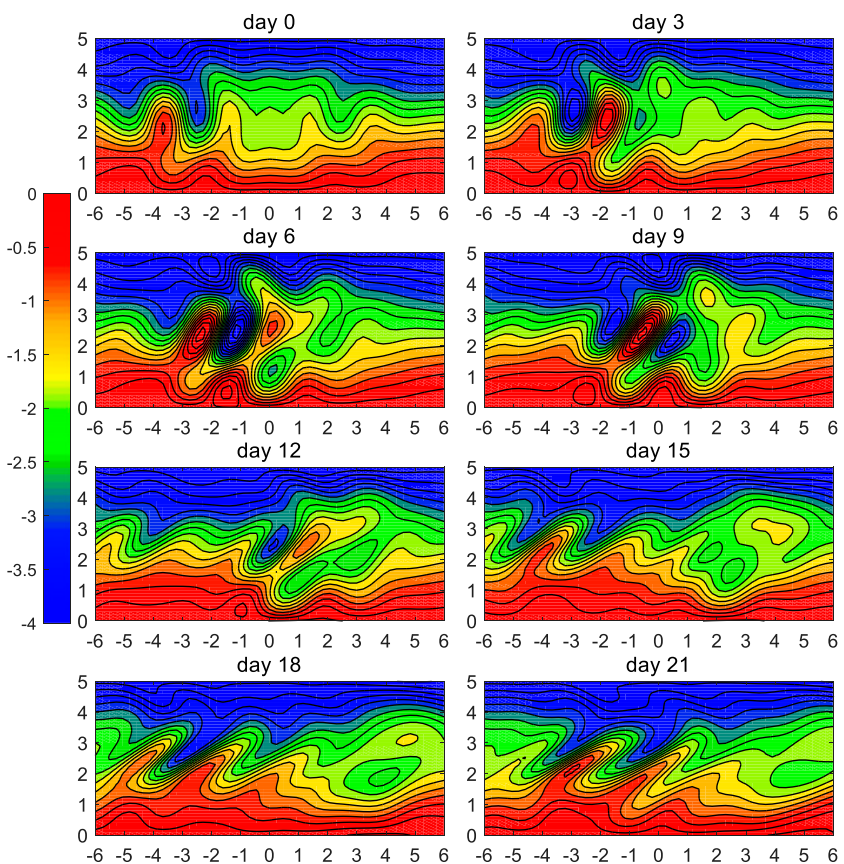

(d) $\psi_{T}$

FIG. 10. As in Fig. 9, but for a strong high-latitude barotropic background westerly wind $(\Delta u=0.2)$ with $U=u_{0}+\Delta u e^{-\gamma\left(y-y_{0}\right)^{2}}$ for $u_{0}=0.7$, $\gamma=0.3$, and $y_{0}=3.75$. 

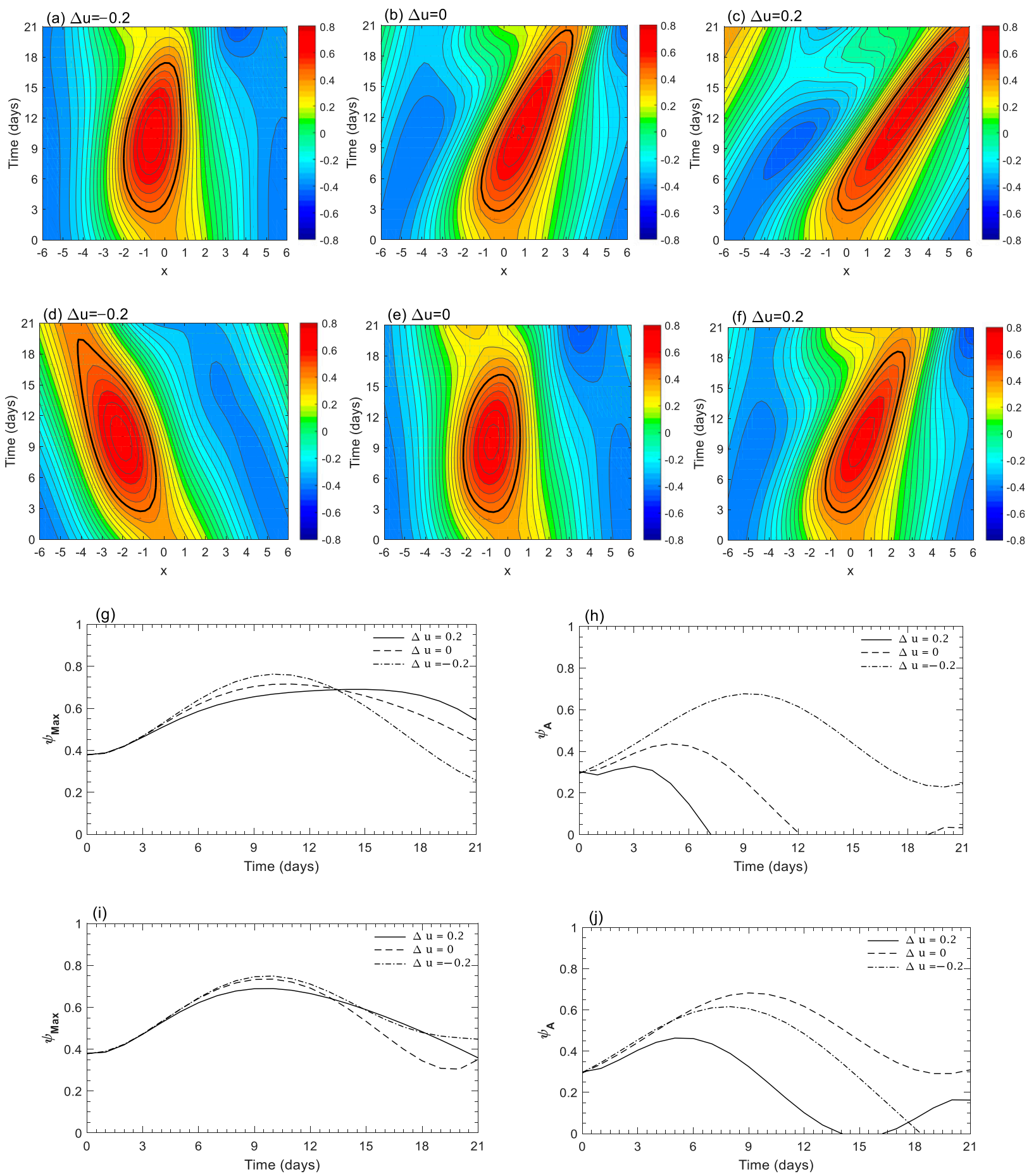

FIG. 11. (a)-(f) Time-longitude evolution of blocking wavy anomaly $\psi_{B}(\mathrm{CI}=0.05$ and 0.45 is marked by the thick black line $)$ at $y=3.75$ and Earth's surface $(z=0)$ of baroclinic blocking $\left(Z_{b}=0.2\right.$ and $\left.F=0.82\right)$ for (a),(d) weak $(\Delta u=-0.2)$ and (c),(f) strong ( $\left.\Delta u=0.2\right)$ barotropic background westerly winds in higher latitudes and (b),(e) uniform barotropic background westerly wind $(\Delta u=0)$ for $U=u_{0}+\Delta u e^{-\gamma\left(y-y_{0}\right)^{2}}$ and $\mathrm{PV}_{y}=\beta-U_{y y}$ with (a)-(c) $u_{0}=0.7$ and (d)-(f) $u_{0}=0.5, \gamma=0.3$, and $y_{0}=3.75$. (g)-(j) Temporal variations of the (g),(i) maximum daily amplitude $\psi_{\text {Max }}$ of blocking anticyclone and (h),(j) domain-averaged daily amplitude $\psi_{A}$ over $-1.5 \leq x \leq-0.5$ and $3.5 \leq y \leq 4$ for $(\mathrm{g}),(\mathrm{h}) u_{0}=0.7$ and (i),(j) $u_{0}=0.5$ with $\Delta u=-0.2$ (dot-dashed line), $\Delta u=0$ (dashed line), and $\Delta u=0.2$ (solid line). 


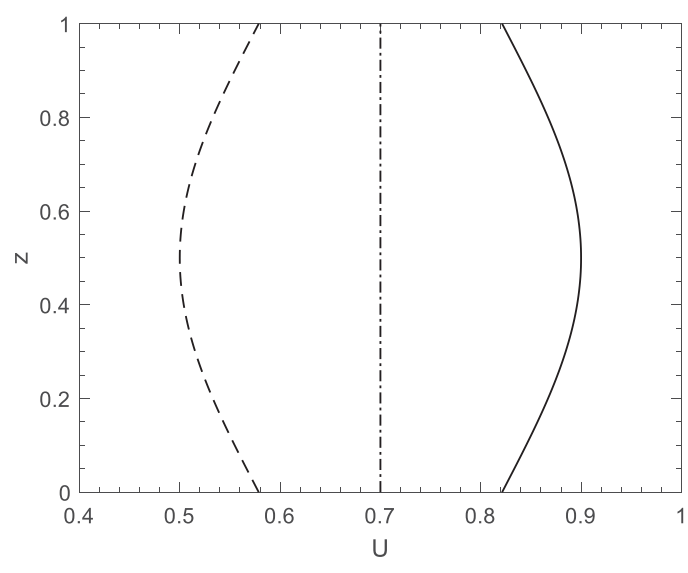

(a)

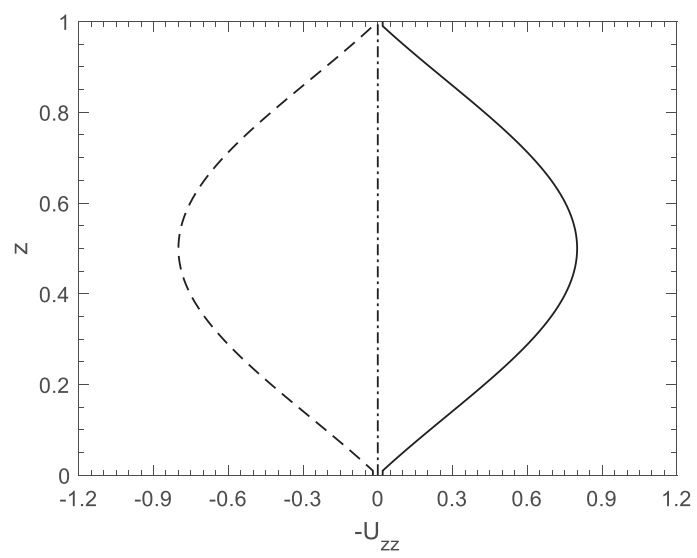

(c)

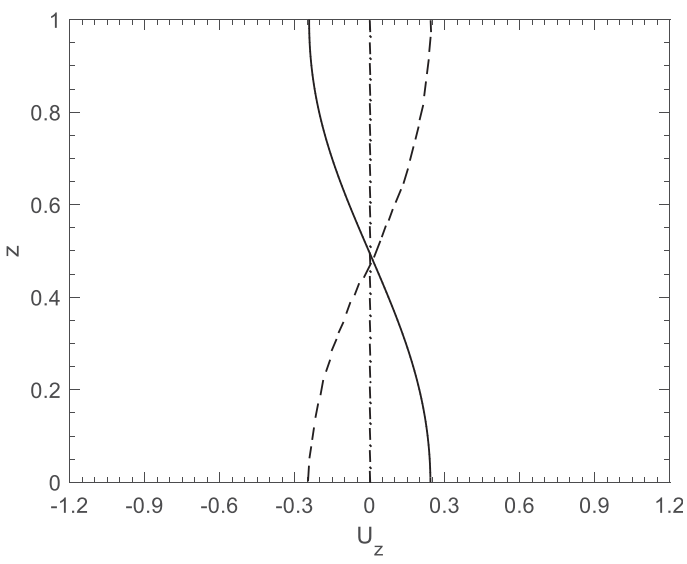

(b)

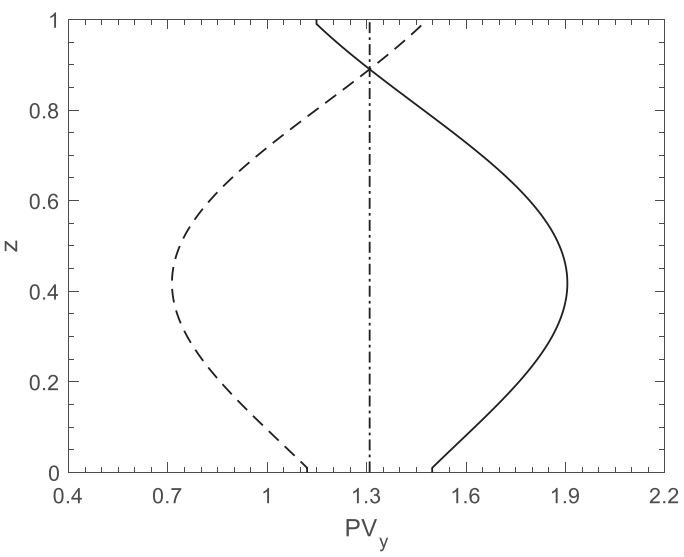

(d)

FIG. 12. Vertical distributions of (a) baroclinic background westerly wind $U(z)=u_{0}+\Delta u e^{-\nu\left(z-z_{0}\right)^{2}}$, (b) $U_{z}$, (c) $-U_{z z}$, and (d) $\mathrm{PV}_{y}=\beta-F_{r} U_{z z}+F_{r} U_{z}$ with $F_{r}=0.72, u_{0}=0.7, \nu=2$, and $z_{0}=0.5$ for $\Delta u=0.2$ (solid line), $\Delta u=$ 0 (dot-dashed line), and $\Delta u=-0.2$ (dashed line).

$u_{0}=0.5$ than for $u_{0}=0.7$ under the $\Delta u=0$ or $\Delta u=0.2$ condition, its local persistence is increased to possess a long local duration (Figs. 11h,j) and a less eastward movement (Figs. 11e,f). Although $\mathrm{PV}_{y}^{D}$ is the same between $u_{0}=0.5$ and $u_{0}=0.7$ for $\Delta u=-0.2$, the lifetime of blocking is slightly longer and its retrogression becomes more evident for $u_{0}=0.5$ (Figs. 11d,i) than for $u_{0}=0.7$ (Figs. 11a,g). Moreover, we also find that the slow decay of eddy-driven blocking in the baroclinic atmosphere with stratification is less distinct than that of eddydriven barotropic blocking (Fig. 7i) under the weak BWW or weak $\mathrm{PV}_{y}^{D}$ condition for $U(y)=u_{0}+\Delta u e^{-\gamma\left(y-y_{0}\right)^{2}}$ with $\gamma=0.3, y_{0}=3.75, u_{0}=0.5$, and $\Delta u=-0.2$. The above results clearly reveal that the eddy-driven blocking tends to have larger amplitude in the midtroposphere and longer lifetime in a baroclinic atmosphere with stratification than in a pure barotropic atmosphere (no vertical variation), whereas the movement and local persistence of blocking can be largely influenced by the magnitude of the barotropic $\mathrm{BWW}$ and $\mathrm{PV}_{y}^{D}$.

\section{c. Impact of baroclinic background westerly wind or $\mathrm{PV}_{y}^{T}$ on eddy-driven blocking}

Here, we further consider a pure baroclinic BWW as $U(z)=U_{T}(z)=u_{0}+\Delta u e^{-\nu\left(z-z_{0}\right)^{2}}$, where $\nu=2$ and $z_{0}=$ 0.5 . For $u_{0}=0.7$, we show the vertical profiles of $U(z)$, $U_{z},-U_{z z}$, and $\mathrm{PV}_{y}=\beta-F_{r} U_{z z}+F_{r} U_{z}\left(F_{r}=0.72\right.$ in Table 1) in Fig. 12 for $\Delta u=0.2, \Delta u=0$, and $\Delta u=-0.2$. In this figure, the baroclinic BWW has no horizontal shear. It is noted that while $U(z)$ and $-U_{z z}$ have a highest or lowest value at $z=0.5$ and $U_{z}$ is zero at $z=0.5$ (Figs. 12a-c), $\mathrm{PV}_{y}$ tends to have a maximum (minimum) amplitude slightly below $z=0.5$ (Fig. 12d) due to the presence of $U_{z}$ (Fig. 12b) for $\Delta u=0.2(\Delta u=-0.2)$. We 
(a) $\Delta \mathrm{u}=-0.2$
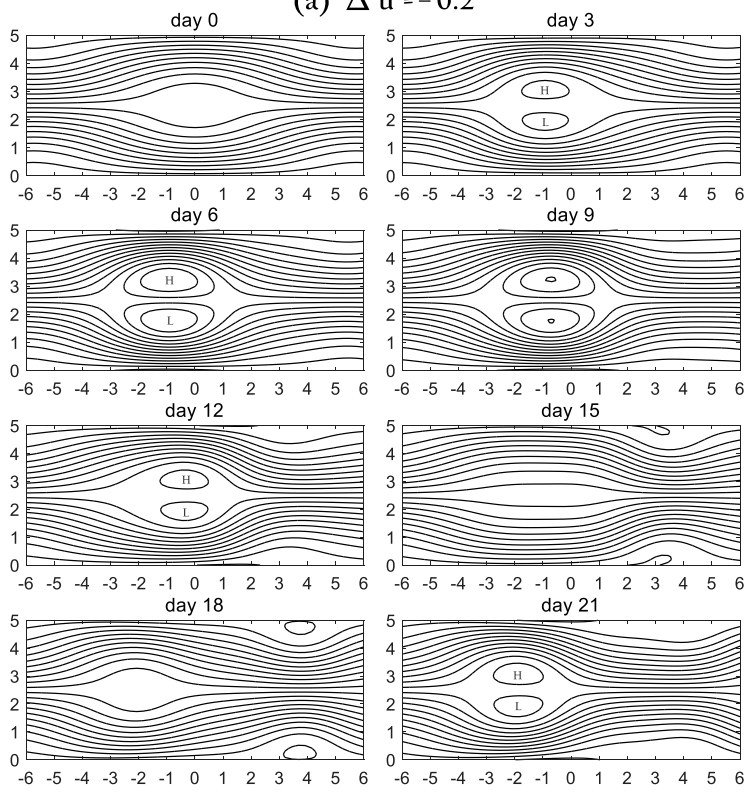

day 9

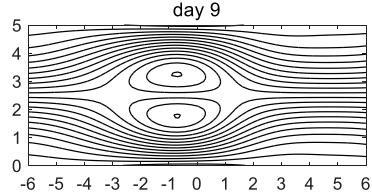

day 15

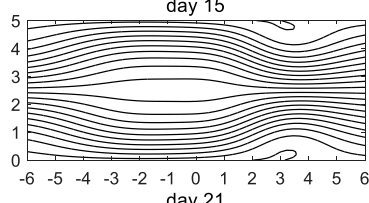

day 21

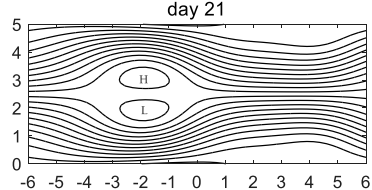

(c) $\Delta u=-0.2$

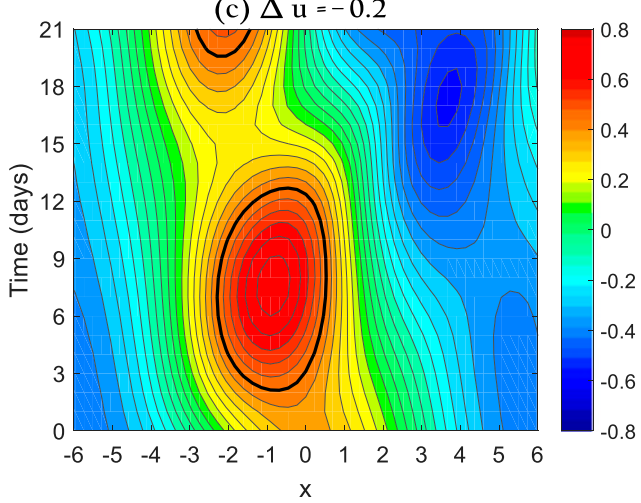

(e)

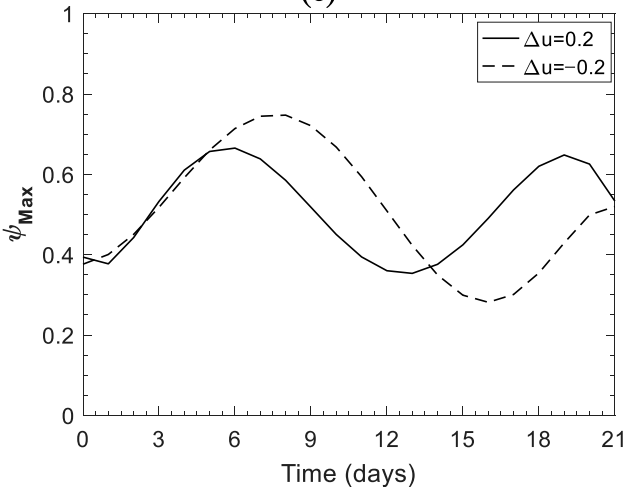

(b) $\Delta \mathrm{u}=0.2$

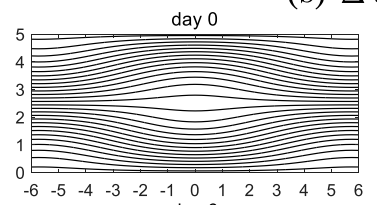

day 6

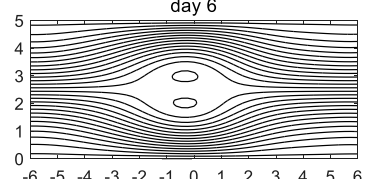

day 12

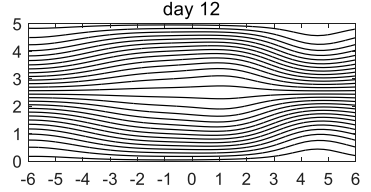

day 18
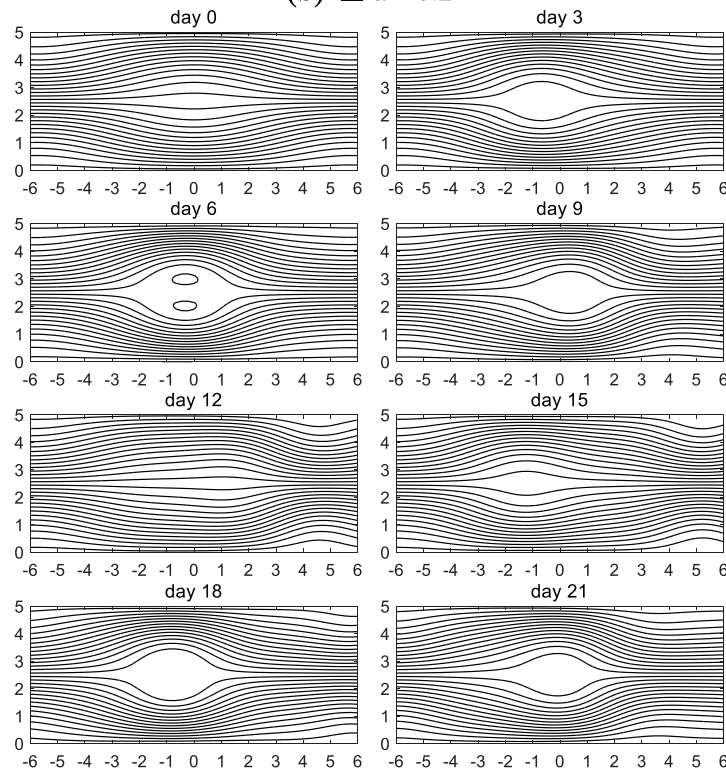

day 9

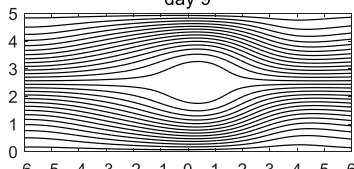

day 15

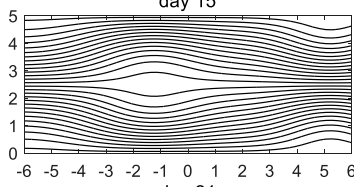

day 21

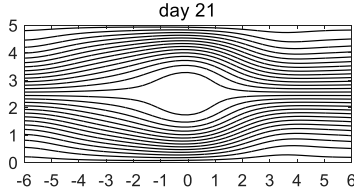

(d) $\Delta u=0.2$

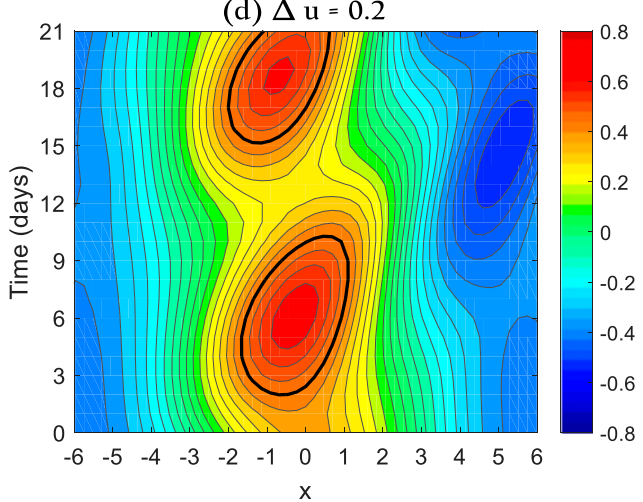

(f)

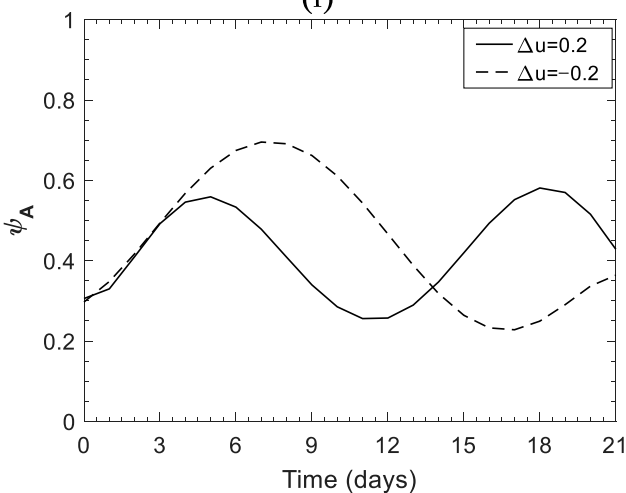

FIG. 13. (a),(b) Instantaneous planetary-scale streamfunction field $\psi_{P}(\mathrm{CI}=0.15)$ of eddy-driven baroclinic blocking with $Z_{b}=0$ and $F=0$ at Earth's surface $(z=0)$ and (c), (d) time-longitude evolution of the blocking wavy anomaly $\psi_{B}(\mathrm{CI}=0.05$ and 0.45 is marked by the thick black line) at $y=3.75$ and $z=0$ under the influence of baroclinic background westerly wind $U(z)=u_{0}+\Delta u e^{-\nu\left(z-z_{0}\right)^{2}}$ and $\mathrm{PV} \mathrm{V}_{y}=$ $\beta-F_{r} U_{z z}+F_{r} U_{z}$ with $F_{r}=0.72, u_{0}=0.7, \nu=2$, and $z_{0}=0.5$ for (a),(c) weak $(\Delta u=-0.2)$ and (b),(d) strong $(\Delta u=0.2)$ baroclinic background westerly winds in the midtroposphere. (e),(f) Temporal variations of the (e) maximum daily amplitude $\psi_{\text {Max }}$ of blocking anticyclone and (f) domain-averaged daily amplitude $\psi_{A}$ over $-1.5 \leq x \leq-0.5$ and $3.5 \leq y \leq 4$ for $\Delta u=-0.2$ (dashed line) and $\Delta u=0.2$ (solid line). 

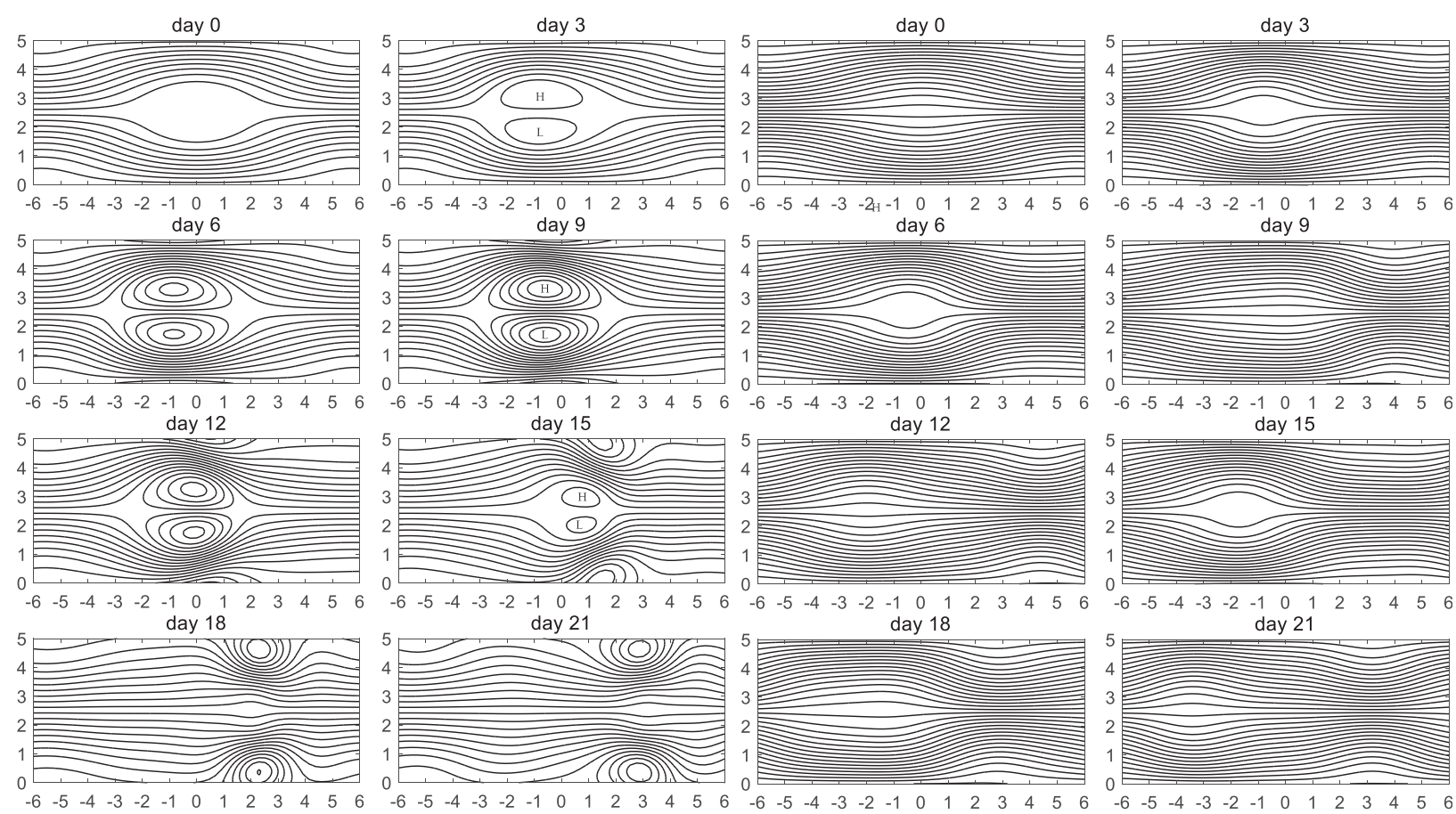

(a) $\Delta u=-0.2$

(b) $\Delta u=0.2$

FIG. 14. (a),(b) Instantaneous planetary-scale streamfunction field $\psi_{P}(\mathrm{CI}=0.15)$ of eddy-driven baroclinic blocking with $Z_{b}=0$ and $F=0$ in the midtroposphere $(z=0.5)$ under the influence of baroclinic background westerly wind $U=u_{0}+\Delta u e^{-\nu\left(z-z_{0}\right)^{2}}$ and $\mathrm{PV}_{y}=$ $\beta-F_{r} U_{z z}+F_{r} U_{z}$ with $F_{r}=0.72, u_{0}=0.7, \nu=2$, and $z_{0}=0.5$ in (a) weak $(\Delta u=-0.2)$ and (b) strong $(\Delta u=0.2)$ baroclinic background westerly winds in the midtroposphere.

also note that $U_{z}$ (Fig. 12b) is much smaller than $-U_{z z}$ (Fig. 12c), thus indicating that $-U_{z z}$ rather than $U_{z}$ plays a major role in the $\mathrm{PV}_{y}$ change. In other words, the vertical variation of MTG with height contributes more importantly to the $\mathrm{PV}_{y}$ change than the MTG itself, because $U_{z}$ represents the value of the MTG. We further find that the change of $\mathrm{PV}_{y}^{T}$ between $\Delta u=-0.2$ and $\Delta u=0.2$ is larger than that of $\mathrm{PV}_{y}^{D}$. Thus, the variation of $\mathrm{PV}_{y}^{T}$ seems to play a larger role in the blocking change than that of $\mathrm{PV}_{y}^{D}$. These results lead us to infer that the vertical variation of the MTG is more important for the blocking change than the MTG strength itself.

As noted above, an eddy-driven blocking can be influenced by $\mathrm{PV}_{y}=\beta-F_{r} U_{z z}+F_{r} U_{z}$ even for $Z_{b}=0$ and $F=0$, when the BWW is a pure baroclinic flow. Here, we first examine this case. We show the planetaryscale streamfunction $\psi_{P}$ field at $z=0$ of eddy-driven blocking for $Z_{b}=0$ and $F=0$ in a baroclinic BWW of $U(z)=u_{0}+\Delta u e^{-\nu\left(z-z_{0}\right)^{2}}$, with $u_{0}=0.7, \nu=2$, and $z_{0}=$ 0.5 in Figs. 13a and 13b for $\Delta u=-0.2$ and $\Delta u=0.2$. The corresponding time-longitude evolution of the blocking wavy anomaly $\psi_{P}$ at $y=3.75$ during the blocking life cycle is shown in Figs. 13c and 13d. It is clearly found that a small $\mathrm{PV}_{y}$ or $\mathrm{PV}_{y}^{T}$ significantly strengthens eddy-driven blocking (Fig. 13a), increases its quasistationarity or local persistence (Fig. 13c) and prolongs its lifetime for $\Delta u=-0.2$. In contrast, a large $\mathrm{PV}_{y}$ or $\mathrm{PV}_{y}^{T}$ for $\Delta u=0.2$ largely reduces the blocking's strength (Fig. 13b), shortens its lifetime and promotes its eastward movement (Fig. 13d). Such a dependence of the lifetime, local persistence, and strength of blocking on the magnitude of baroclinic BWW or $\mathrm{PV}_{y}^{T}$ can be further seen from the time variations of $\psi_{\text {Max }}$ and $\psi_{A}$ as shown in Figs. 13e and 13f. Clearly, less local persistence of blocking is evident for $\Delta u=0.2$ in Fig. 13f.

For $\Delta u=-0.2$ or a small $\mathrm{PV}_{y}^{T}$, the eddy-driven blocking is much stronger in the midtroposphere $(z=0.5)$ than in Earth's surface $(z=0)$ (Fig. 14a). In contrast, the blocking has shorter lifetime and is much weaker in the midtroposphere than in Earth's surface for $\Delta u=0.2$ or a large $\mathrm{PV}_{y}^{T}$ (Fig. 14b). This means that a small $\mathrm{PV}_{y}^{T}$ environment tends to favor the eddy-driven blocking especially in the midtroposphere, whereas a large $\mathrm{PV}_{y}^{T}$ tends to strongly inhibit the blocking in 


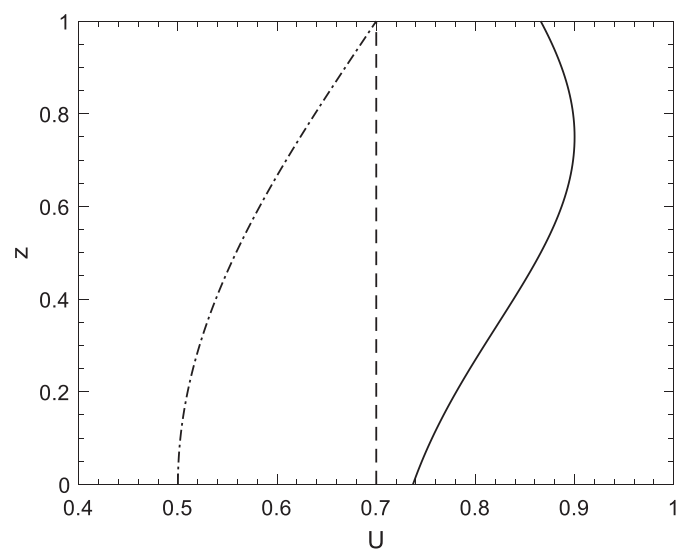

(a)

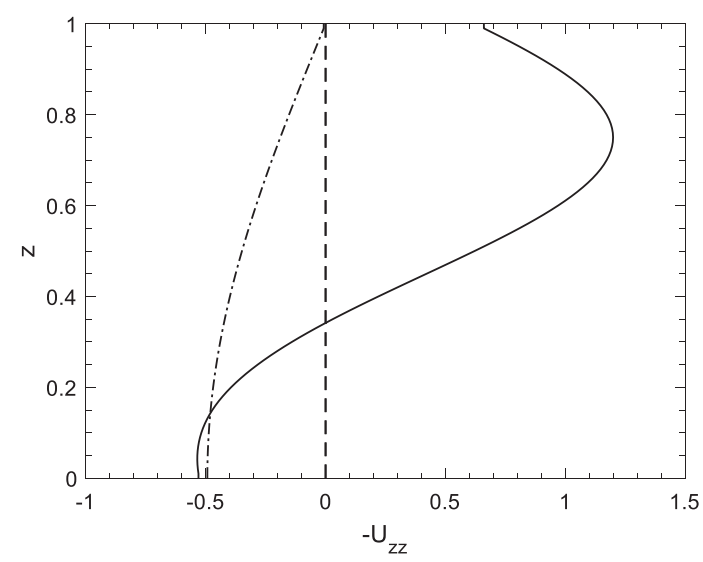

(c)

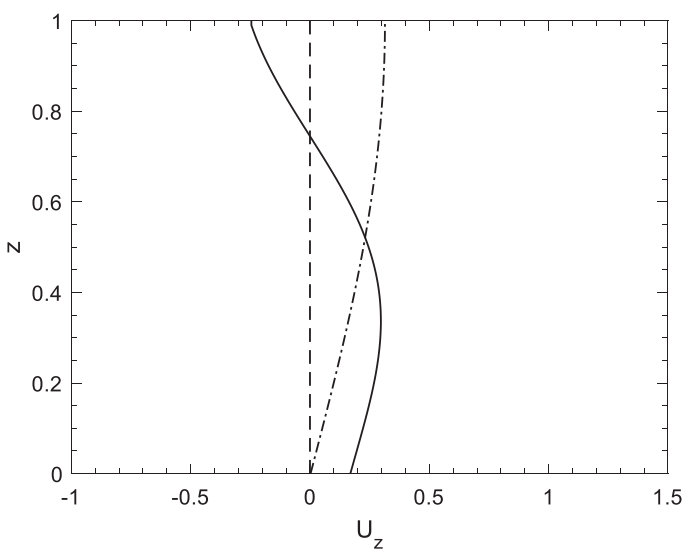

(b)

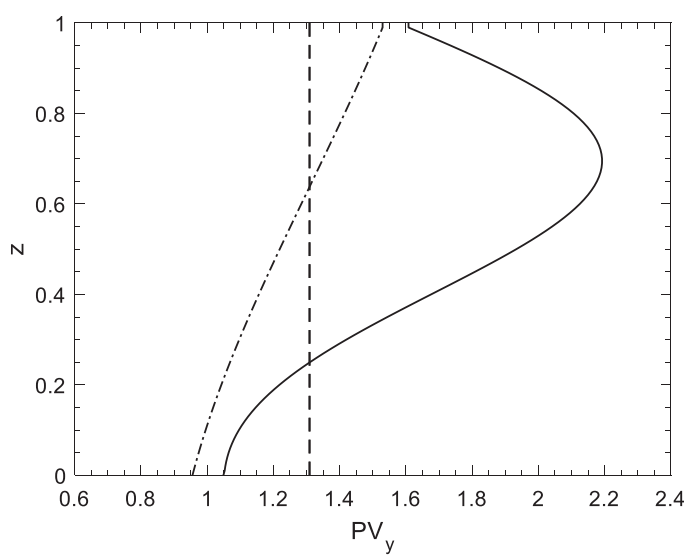

(d)

FIG. 15. Vertical distributions of (a) baroclinic background westerly wind $U(z)$, (b) $U_{z}$, (c) $-U_{z z}$, and (d) $\mathrm{PV}_{y}=\beta-F_{r} U_{z z}+F_{r} U_{z}$ with $F_{r}=0.72$ for $U(z)=u_{0}+\Delta u e^{-\nu\left(z-z_{0}\right)^{2}}$ with $u_{0}=0.7, \nu=3, z_{0}=0.75$, and $\Delta u=$ 0.2 (solid line), $U=u_{0}=0.7$ (dashed line), and $U(z)=u_{0}+\Delta u \cos (\pi z / 2)$ with $u_{0}=0.7$ and $\Delta u=-0.2$ (dot-dashed line).

lifetime and strength. Here, we further examine the effect of the BWW change mainly in the lower troposphere on eddy-driven blocking. Figure 15 shows the variations of $U(z), U_{z},-U_{z z}$, and $\mathrm{PV}_{y}=\beta-F_{r} U_{z z}+$ $F_{r} U_{z}$ with height for $U(z)=u_{0}+\Delta u e^{-\nu\left(z-z_{0}\right)^{2}}$ with $u_{0}=$ $0.7, \nu=3, z_{0}=0.75$, and $\Delta u=0.2$ (solid line), $U=u_{0}$ with $u_{0}=0.7$ (dashed line) and $U(z)=u_{0}+\Delta u \cos (\pi z / 2)$ with $u_{0}=0.7$ and $\Delta u=-0.2$ (dot-dashed line). It is seen that while the baroclinic BWW (Fig. 15a) is weaker for $U(z)=u_{0}+\Delta u e^{-\nu\left(z-z_{0}\right)^{2}}$ and $U(z)=u_{0}+$ $\Delta u \cos (\pi z / 2)$ in the lower troposphere than in the upper troposphere, it is stronger (weaker) for $U(z)=u_{0}+\Delta u e^{-\nu\left(z-z_{0}\right)^{2}}\left[U(z)=u_{0}+\Delta u \cos (\pi z / 2)\right]$ than $U=u_{0}$. In addition, we note that $\mathrm{PV}_{y}$ is smaller in the lower troposphere for $U(z)=u_{0}+\Delta u e^{-\nu\left(z-z_{0}\right)^{2}}$ and $U(z)=u_{0}+\Delta u \cos (\pi z / 2)$ (Fig. 15d), but larger for $U(z)=u_{0}+\Delta u e^{-\nu\left(z-z_{0}\right)^{2}}$ than for $U(z)=u_{0}+$
$\Delta u \cos (\pi z / 2)$. For the two types of baroclinic BBWs, we show the time-longitude evolution of the blocking wavy anomaly $\psi_{P}$ at $y=3.75$ and $z=0$ during the blocking life cycle and the time series of their corresponding daily $\psi_{\mathrm{Max}}$ and $\psi_{A}$ in Fig. 16 for $U(z)=u_{0}+\Delta u e^{-\nu\left(z-z_{0}\right)^{2}}$ and $U(z)=u_{0}+\Delta u \cos (\pi z / 2)$. It is also found that when $\mathrm{PV}_{y}$ is small in the lower troposphere, the eddy-driven blocking can have longer lifetime and larger strength (Figs. 16a,b) than for $U=0.7$ (Fig. 7b), even though the BWW is weaker for $U=0.7$ than for $U(z)=u_{0}+\Delta u e^{-\nu\left(z-z_{0}\right)^{2}}$. Such blocking features can also be seen from Fig. 16c. The above results clearly indicate that a small $\mathrm{PV}_{y}$ in the lower troposphere is favorable for the long lifetime and large strength of eddy-driven blocking. However, we find that the movement of eddy-driven blocking depends on the strength and vertical profile of the 


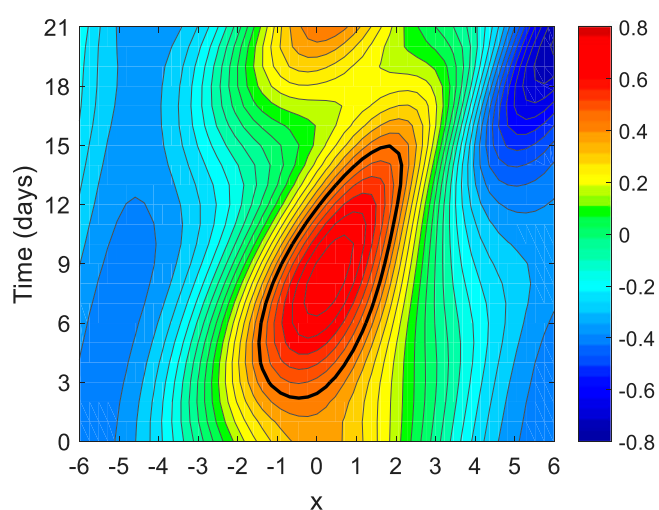

(a)

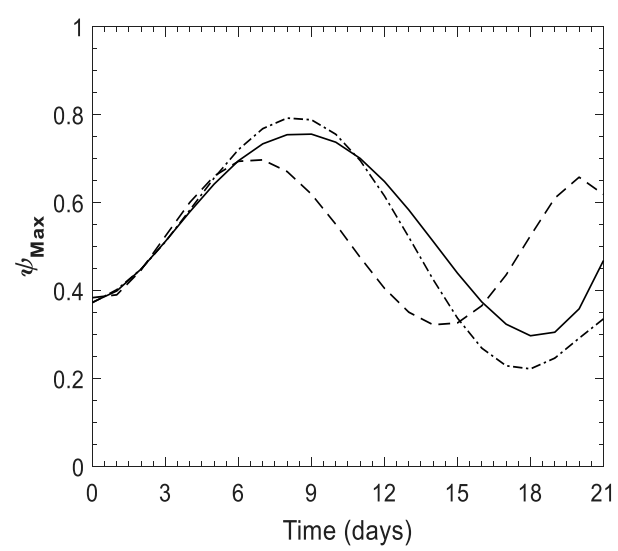

(c)

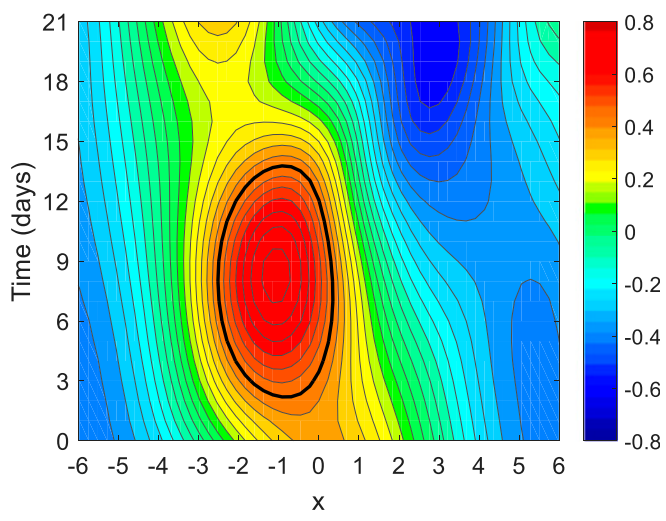

(b)

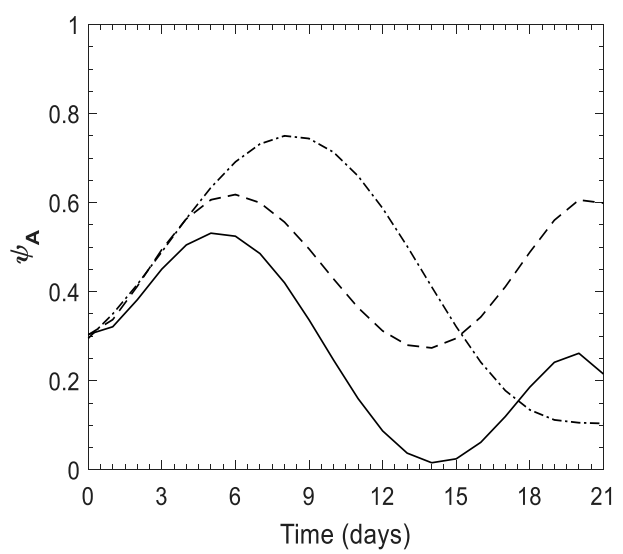

(d)

FIG. 16. (top) Time-longitude evolution of the blocking wavy anomaly $\psi_{B}$ (CI $=0.05$ and 0.45 is marked by the thick black line) with $Z_{b}=0$ and $F=0$ at $y=3.75$ and $z=0$ during the blocking life cycle under the influence of $\mathrm{PV}_{y}=\beta-F_{r} U_{z z}+F_{r} U_{z}$ with $F_{r}=0.72$ and different baroclinic background westerly winds in Fig. 15 for (a) $U(z)=u_{0}+\Delta u e^{-\nu\left(z-z_{0}\right)^{2}}$ with $u_{0}=0.7, \nu=3, z_{0}=0.75$, and $\Delta u=0.2$; and (b) $U(z)=u_{0}+\Delta u$ $\cos (\pi z / 2)$ with $u_{0}=0.7$ and $\Delta u=-0.2$. (bottom) Temporal variations of the (c) maximum daily amplitude $\psi_{\text {Max }}$ of blocking anticyclone and (d) domain-averaged daily amplitude $\psi_{A}$ over $-1.5 \leq x \leq-0.5$ and $3.5 \leq y \leq$ 4 during the blocking life cycle for $U(z)=u_{0}+\Delta u e^{-\nu\left(z-z_{0}\right)^{2}}$ with $u_{0}=0.7, \nu=3, z_{0}=0.75$, and $\Delta u=0.2$ (solid line), $U=u_{0}$ with $u_{0}=0.7$ (dashed line), and $U(z)=u_{0}+\Delta u \cos (\pi z / 2)$ with $u_{0}=0.7$ and $\Delta u=-0.2$ (dot-dashed line).

baroclinic BWW. While the blocking moves eastward for $U(z)=u_{0}+\Delta u e^{-\nu\left(z-z_{0}\right)^{2}}$, it is less mobile for $U(z)=$ $u_{0}+\Delta u \cos (\pi z / 2)$. In this case, $U(z)=u_{0}+\Delta u \cos (\pi z / 2)$ $\left[U(z)=u_{0}+\Delta u e^{-\nu\left(z-z_{0}\right)^{2}}\right]$ corresponds to an eddydriven blocking with a long (short) local persistence (Fig. 16d). When the baroclinic BWW is small (large) in the upper (lower) troposphere, the eddy-driven blocking is greatly suppressed because of the presence of a large $\mathrm{PV}_{y}$ in the lower to midtroposphere (not shown).

We further repeat the same calculation as in Fig. 13 for $Z_{b}=0.2$ and $F=0.82$, and show the calculation results in Fig. 17. Clearly, the eddy-driven blocking has longer lifetime in a baroclinic atmosphere with stratification $\left(Z_{b}=0.2\right.$ and $\left.F=0.82\right)$ (Figs. 17a,b) than in a barotropic atmosphere without the effect of stratification $\left(Z_{b}=0\right.$ and $\left.F=0\right)$ (Figs. 13a,b), even though the $\mathrm{PV}_{y}$ field includes the effect of stratification $\left(F_{r} \neq 0\right)$. It is further found that while the blocking has larger strength (Fig. 17a) and less eastward movement (Fig. 17c) for a small $\mathrm{PV}_{y}^{T}$ with $\Delta u=-0.2$ than for a large $\mathrm{PV}_{y}^{T}$ with $\Delta u=0.2$ (Figs. 17b,d), it shows slower decay for a large $\mathrm{PV}_{y}^{T}$ with $\Delta u=0.2$ (solid line in Fig. 17e). Moreover, the increased local persistence of eddydriven blocking is more evident for a small $\mathrm{PV}_{y}^{T}$ with $\Delta u=-0.2$ than for a large $\mathrm{PV}_{y}^{T}$ with $\Delta u=0.2$ (Fig. 17f), 
(a) $\Delta \mathrm{u}=-0.2$

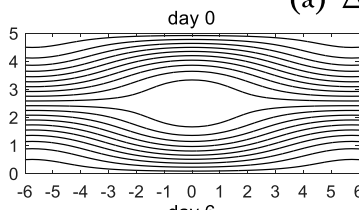

day 6

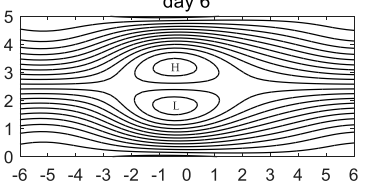

day 12

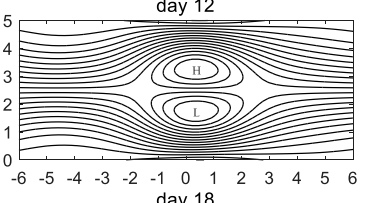

day 18
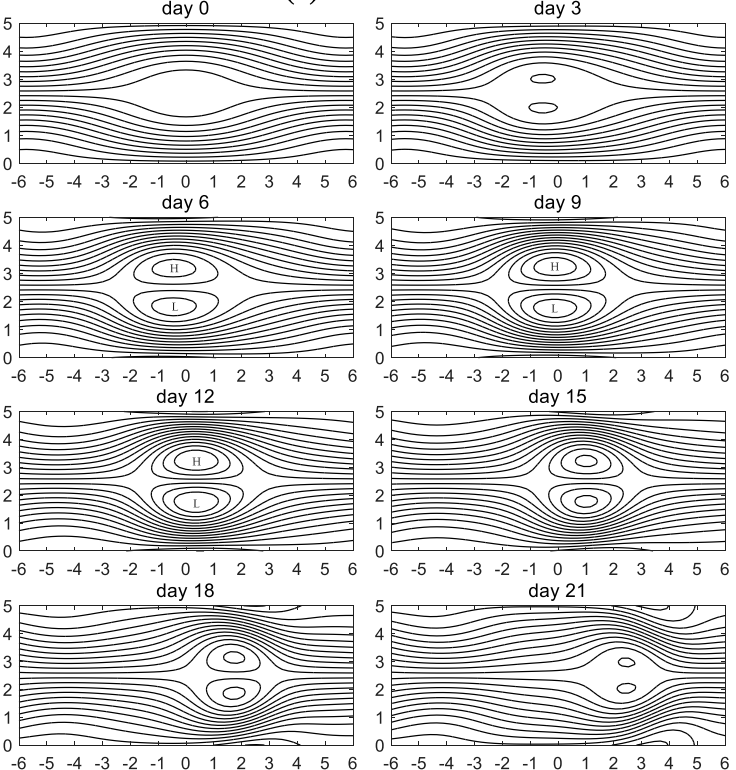

day 9

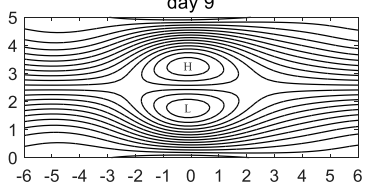

day 15

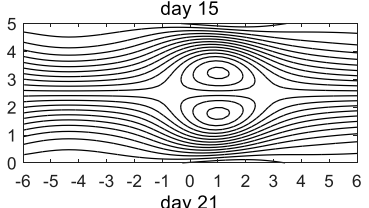

day 21

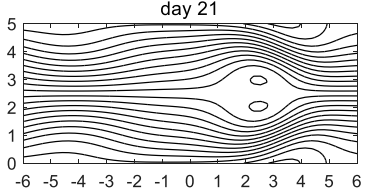

(c) $\Delta u=-0.2$

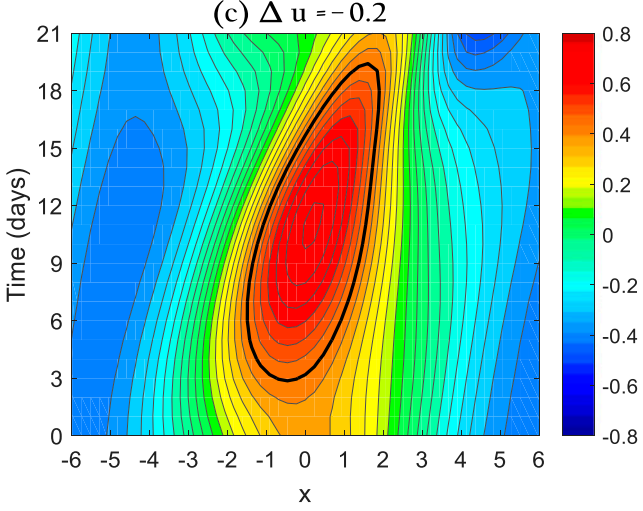

(e)

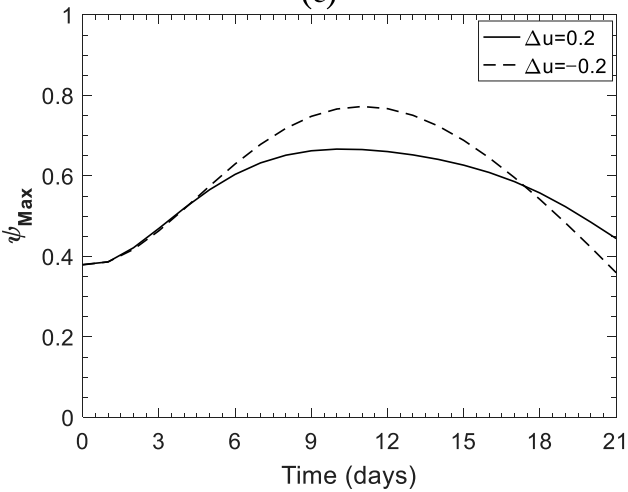

(b) $\Delta \mathrm{u}=0.2$
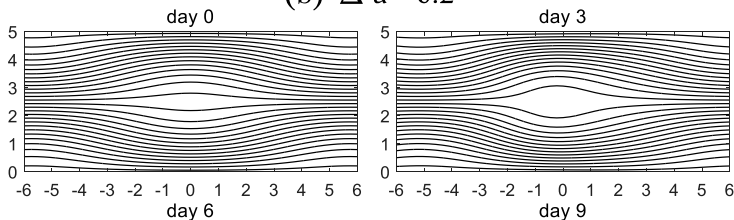

day 9

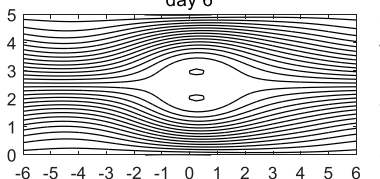

day 12

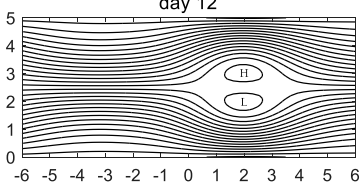

day 18
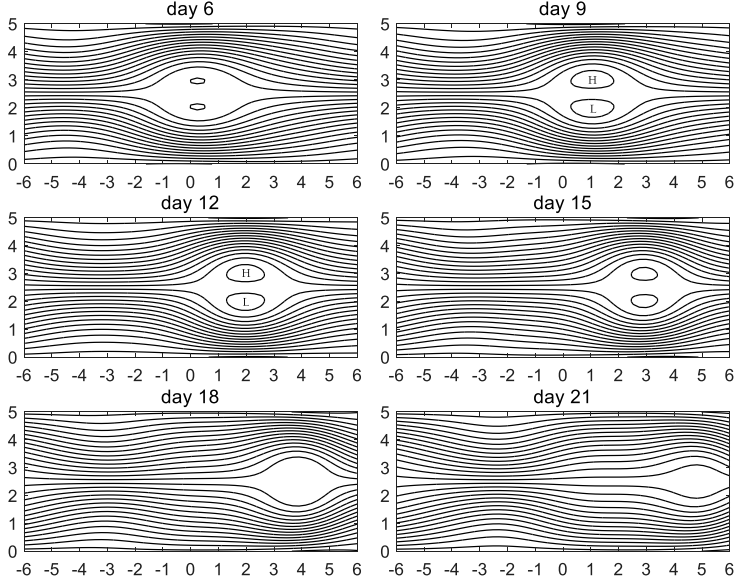

day 15

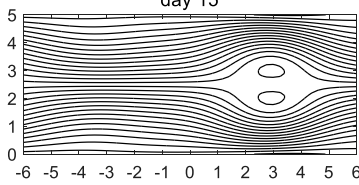

day 21

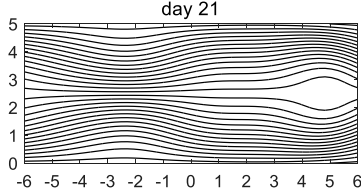

(d) $\Delta u=0.2$

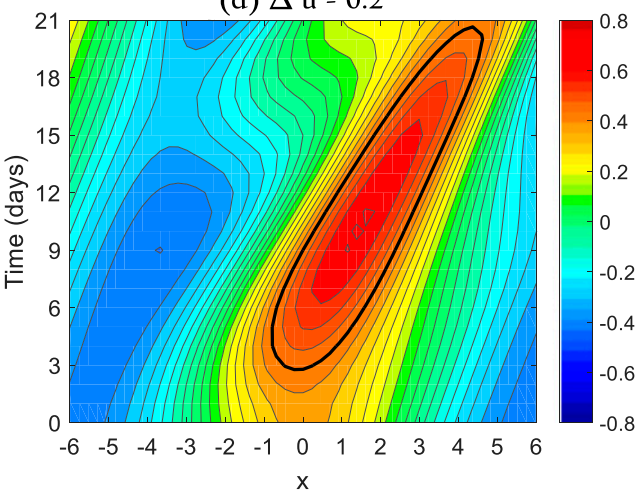

(f)

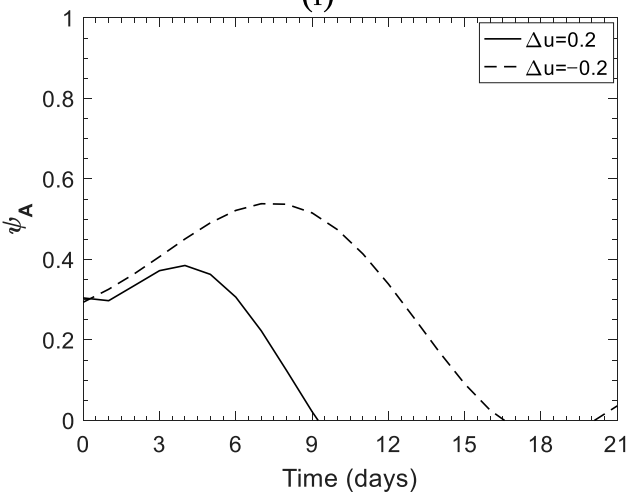

FIG. 17. (a),(b) Instantaneous planetary-scale streamfunction field $\psi_{P}(\mathrm{CI}=0.15)$ of eddy-driven baroclinic blocking $\left(Z_{b}=0.2\right.$ and $F=$ $0.82)$ at Earth's surface $(z=0)$ and (c), (d) time-longitude evolution of the blocking wavy anomaly $\psi_{B}(\mathrm{CI}=0.05$ and 0.45 is marked by the thick black line) at $y=3.75$ and $z=0$ under the influence of baroclinic background westerly wind $U=u_{0}+\Delta u e^{-\nu\left(z-z_{0}\right)^{2}}$ and $\mathrm{PV} y=\beta-$ $F_{r} U_{z z}+F_{r} U_{z}$ with $F_{r}=0.72, u_{0}=0.7, \nu=2$, and $z_{0}=0.5$ for (a),(c) weak $(\Delta u=-0.2)$ and (b),(d) strong $(\Delta u=0.2)$ baroclinic background westerly winds in the middle troposphere. (e),(f) Temporal variations of the (e) maximum daily amplitude $\psi_{\text {Max }}$ of blocking anticyclone and (f) domain-averaged daily amplitude $\psi_{A}$ over $-1.5 \leq x \leq-0.5$ and $3.5 \leq y \leq 4$ for $\Delta u=-0.2$ (dashed line) and $\Delta u=0.2$ (solid line). 
though the blocking has long lifetime for $\Delta u=-0.2$ and $\Delta u=0.2$ (Figs. 17c,d).

We also calculate a weak baroclinic BWW case with $u_{0}=0.5$ for the same parameters as in Fig. 17 and show the results in Fig. 18. The results similar to those in Fig. 17 are found for $u_{0}=0.5$, but the slow decay of blocking is almost invisible for a large $\mathrm{PV}_{y}^{T}$ with $\Delta u=0.2$. Specifically, it is found that the eddy-driven blocking has longer lifetime, larger strength and stronger local persistence for a small $\mathrm{PV}_{y}^{T}$ with $\Delta u=-0.2$ (Figs. 18a,c,e,f) than for a large $\mathrm{PV}_{y}^{T}$ with $\Delta u=0.2$ (Figs. 18b, d,e,f). Overall, the weak baroclinic BWW and small $\mathrm{PV}_{y}^{T}$ in a baroclinic atmosphere with stratification tend to favor increased local persistence (or reduced eastward movement), large amplitude and long lifetime of eddy-driven blocking, thus promoting a long-lived meandering westerly jet stream in a fixed region.

Since $\mathrm{PV}_{v}^{T}$ with $\Delta u=-0.2$ represents the effect of reduced MTG, it is thought that the reduced MTG favors increased local persistence and large amplitude of blocking or a persistent meandering of westerly jet streams. Naturally, our nonlinear multiscale theory here can apply to the effects of AA (or sea ice decline), Eurasian snow cover, and internal low-frequency variability on the winter midlatitude blocking and cold extremes in terms of the MTG and zonal wind changes (Newson 1973; Luo et al. 2018; Cohen et al. 2020; Li and Luo 2019). Further study along this direction will be reported in another paper.

\section{Conclusions and discussion}

In this paper, the nonlinear multiscale interaction (NMI) model of atmospheric blocking proposed by Luo $(2000,2005)$ and Luo et al. $(2014,2019)$ and Zhang and Luo (2020) has been extended to include a three-dimensional background flow to examine how the horizontal or vertical shear of a threedimensional background flow influences the spatiotemporal evolution of eddy-driven blocking. In the extended NMI model, the eddy-driven blocking is considered as a nonlinear Rossby wave packet governed by a forced NLS equation (Luo 2000, 2005), which is initiated by preexisting synoptic-scale eddies upstream as a nonlinear initial-value problem as in Luo et al. (2019). While this model emphasizes the key role of preexisting incident synoptic-scale eddies in the life cycle of blocking, it considers the eddy straining or CWB as a result of the feedback of intensified blocking on preexisting synoptic-scale eddies.
In this model, the spatiotemporal evolution of eddydriven blocking does not only depend on the parameters of the incipient block and preexisting synoptic-scale eddies, but also on the background field. Although the basic winds and meridional temperature gradient (MTG) can be considered as a background field, the two parameters were not unified into a single factor influencing the evolution of blocking in the previous models. In the present paper, we have unified the background wind and MTG into the background meridional $\mathrm{PV}$ gradient $\left(\mathrm{PV}_{y}\right)$ as a single factor affecting the temporal evolution of blocking. Because the lifetime, movement and strength of blocking are significantly influenced by the magnitude of $\mathrm{PV}_{y}$, one can quantify the different roles of the dynamical PV gradient $\left(\mathrm{PV}_{y}^{D}\right)$ and thermodynamic $\mathrm{PV}$ gradient $\left(\mathrm{PV}_{y}^{T}\right)$ in the spatiotemporal evolution of eddy-driven blocking by dividing $\mathrm{PV}_{y}$ into $\mathrm{PV}_{y}=\beta+\mathrm{PV}_{y}^{D}+\mathrm{PV}_{y}^{T}$. In this model, $\mathrm{PV}_{y}^{D}=V_{x y}-U_{y y}$ is related to the horizontal (mainly meridional) shear of background westerly wind (BWW), whereas $\mathrm{PV}_{y}^{T}=F_{r} U_{z}-F_{r} U_{z z}$ reflects the MTG (or vertical shear of the BWW) and its vertical variation with height.

On the other hand, because the energy dispersion, nonlinearity strength and preexisting eddy forcing in the blocking wave packet equation depend on the values of $F, \mathrm{PV}_{y}^{D}$, and $\mathrm{PV}_{y}^{T}$, the evolution of blocking is inevitably influenced by the atmospheric stratification, the horizontal shear of the BWW, and its vertical shear (or MTG). Using the extended NMI model, we find that in a pure barotropic atmosphere without stratification effect $\left(Z_{b}=0\right.$ and $\left.F=0\right)$ the eddy-driven barotropic blocking has a short lifetime less than 15 days, whose spatial pattern or horizontal orientation is dominated by the magnitude and meridional distribution of $\mathrm{PV}_{y}^{D}$. Only when the barotropic BWW is weaker especially in higher latitudes, the eddy-driven barotropic blocking can have longer lifetime, more notable retrogression and slower decay due to smaller $\mathrm{PV}_{y}^{D}$ especially in higher latitudes.

In a baroclinic atmosphere with stratification (e.g., $Z_{b}=0.2$ and $F=0.82$ ), the eddy-driven baroclinic blocking tends to have long lifetime near 20 days and large amplitude in the midtroposphere even under the vanishing of $\mathrm{PV}_{y}^{T}$. The eddy-driven blocking exhibits longer lifetime, more notable eastward movement, slower decay, and shorter local persistence, when the barotropic $\mathrm{BWW}$ or $\mathrm{PV}_{y}^{D}$ with a maximum strength in higher latitudes is larger. In contrast, when the barotropic $\mathrm{BWW}$ or $\mathrm{PV}_{y}^{D}$ with a minimum strength in higher latitudes is smaller, the eastward movement of blocking is suppressed to strengthen its local persistence or increase its duration in a fixed region. In this case, the slow 
(a) $\Delta \mathrm{u}=-0.2$
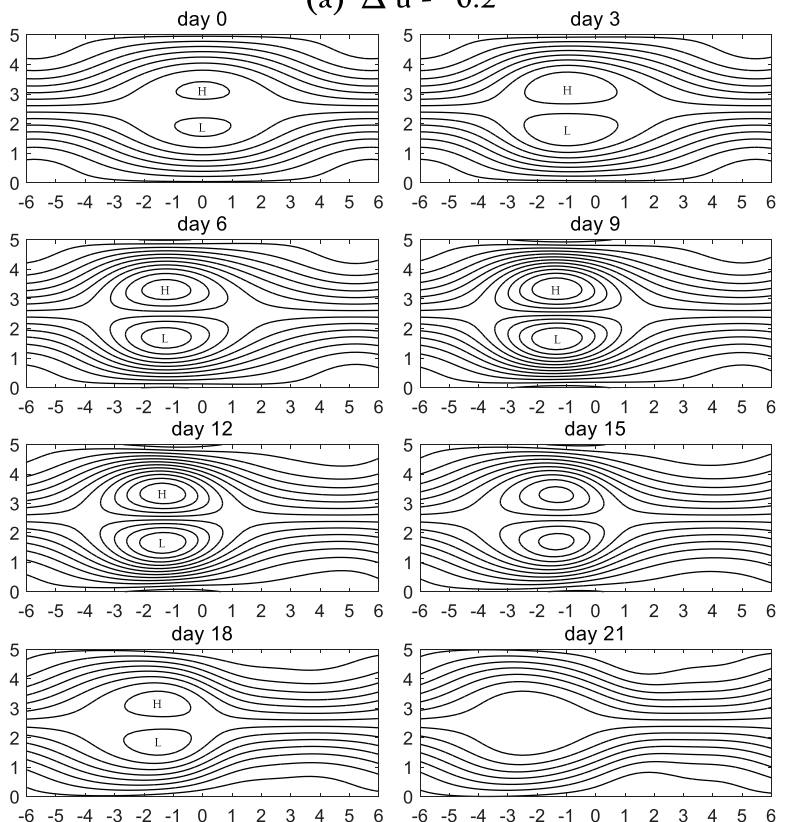

day 9

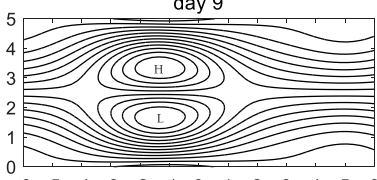

$\begin{array}{lll}-1 & 0 \\ & & 15\end{array}$

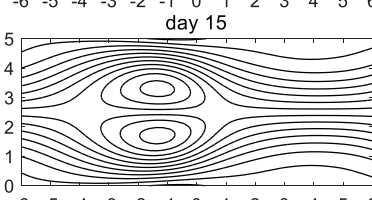

day 21

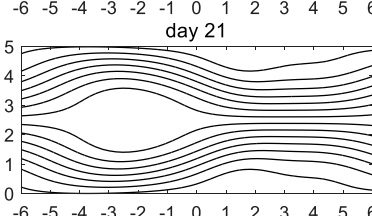

(c) $\Delta \mathrm{u}=-0.2$

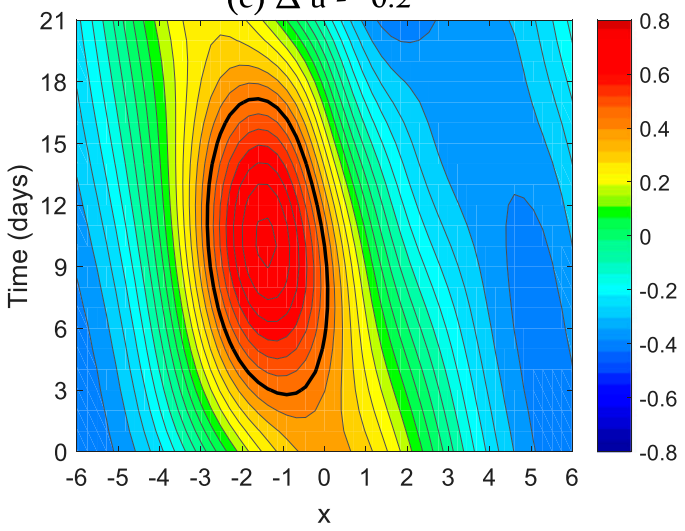

(e)

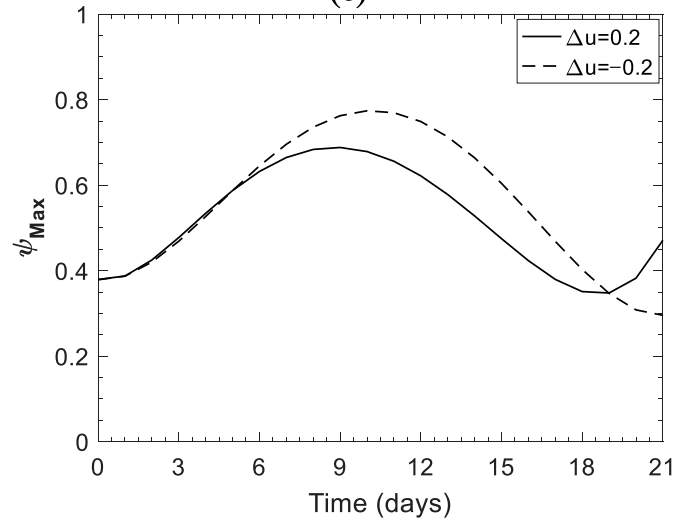

(b) $\Delta u=0.2$

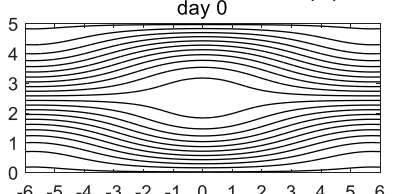

day 6

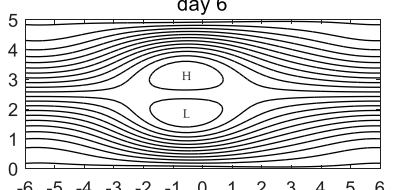

day 12

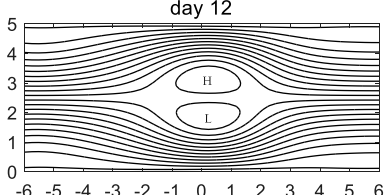

day 18
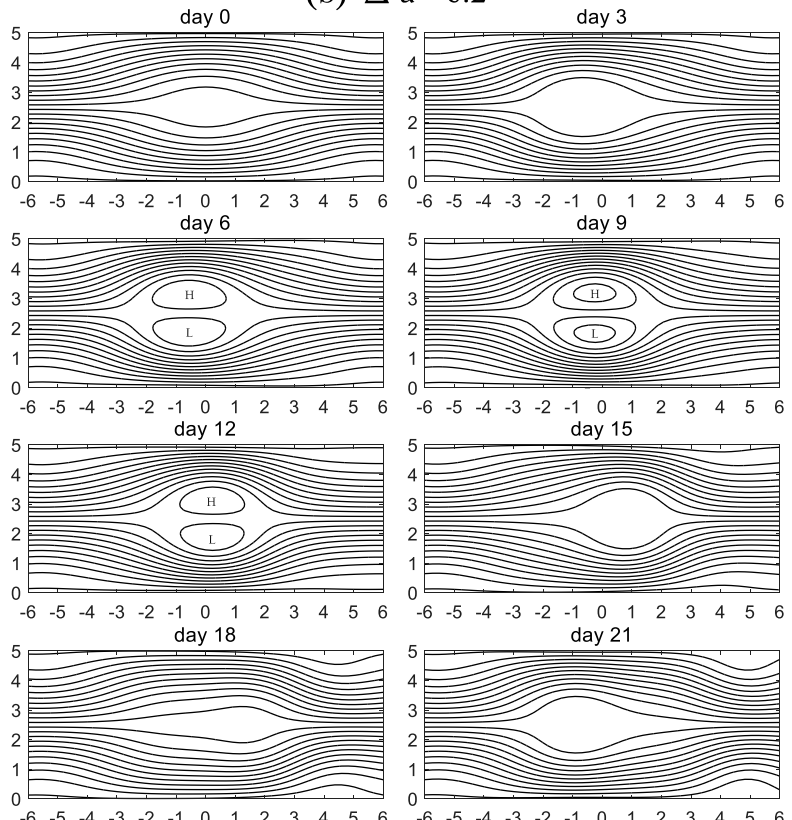

day 9

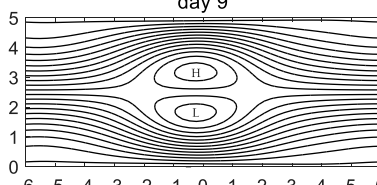

day 15

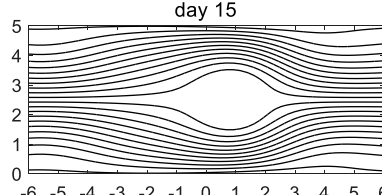

day 21

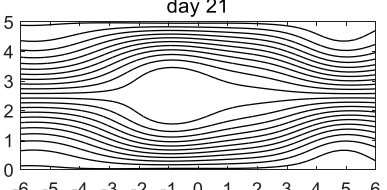

(d) $\Delta u=0.2$

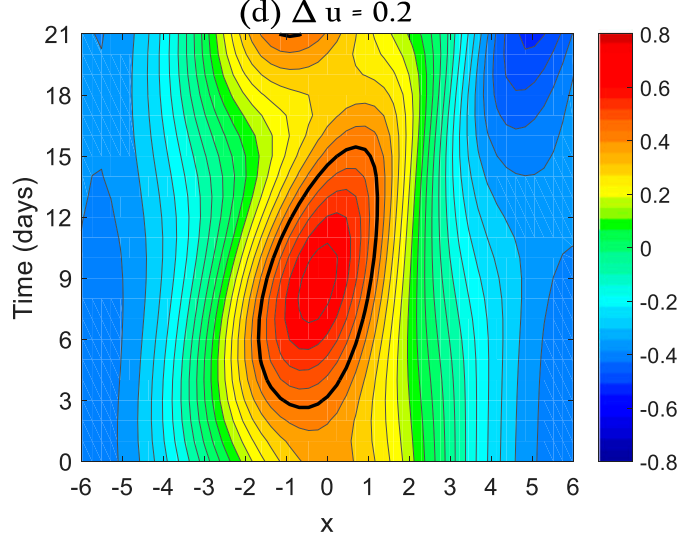

(f)

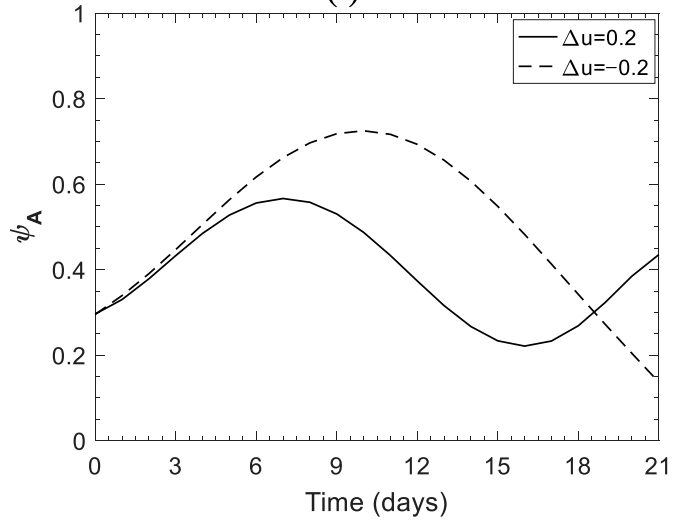

FIG. 18. As in Fig. 17, but for a baroclinic background westerly wind with a weak uniform westerly wind $u_{0}=0.5$ of $U=u_{0}+\Delta u e^{-\nu\left(z-z_{0}\right)^{2}}$, $\mathrm{PV}_{y}=\beta+\mathrm{PV}_{y}^{T}$, and $\mathrm{PV}_{y}^{T}=F_{r} U_{z}-F_{r} U_{z z}$ for $F_{r}=0.72, \nu=2$, and $z_{0}=0.5$. 
decay of eddy-driven blocking is less evident than that in a pure barotropic case.

We also examined the impact of baroclinic BWW or $\mathrm{PV}_{y}^{T}$ on the temporal evolution of eddy-driven blocking under $\mathrm{PV}_{y}^{D}=0$. It is found that $\mathrm{PV}_{y}^{T}$ has a large impact on the movement, lifetime, and strength of eddy-driven blocking in the baroclinic atmosphere with stratification. When the baroclinic $\mathrm{BWW}$ or $\mathrm{PV}_{y}^{T}$ is small in the midtroposphere or in lower troposphere, the eddy-driven blocking can have large amplitude, long lifetime, weak movement, and strong local persistence. In particular, we find that the change of $\mathrm{PV}_{y}^{T}$ plays a larger role in the variation of blocking in strength, movement, and duration because the $\mathrm{PV}_{y}^{T}$ change of $\mathrm{PV}_{y}$ is larger than its $\mathrm{PV}_{y}^{D}$ change. In addition, a new finding is presented that under some background flow conditions the vertical variation of MTG contributes likely more importantly to the $\mathrm{PV}_{y}^{T}$ change than the MTG itself, thus suggesting that the vertical variation of MTG is more important for the blocking change than the MTG itself for some cases. In the further study, we should consider the effect of the vertical variation of MTG on atmospheric blocking in addition to considering the role of MTG (or $U_{z}$ ) Thus, the magnitude of $\mathrm{PV}_{y}$ including $U_{z}$ and $U_{z z}$ is an appropriate parameter describing the change of blocking.

Moreover, it is worthy of noting that our theory here does not apply to the real case. For example, how a changing climate (i.e., changes in AA and SST anomalies in the North Pacific and North Atlantic) influences the blocking variability through changing $\mathrm{PV}_{y}^{D}$ and $\mathrm{PV}_{y}^{T}$ in mid-high latitudes is not discussed in this paper. This problem deserves a further study, which will be examined in the future work.

Acknowledgments. This research was supported by the National Key Research and Development Program of China (2016YFA0601802), the Chinese Academy of Sciences Strategic Priority Research Program (Grant XDA19070403), and the National Science Foundation of China (Grants 41790473 and 41430533).

\section{APPENDIX A}

\section{Coefficients of Deformed Eddies in Eq. (6g) in the Extended NMI Model}

The coefficients of deformed eddies in Eq. $(6 \mathrm{~g})$ are defined as

$$
\begin{gathered}
p_{j}=\frac{\left(k-2 \tilde{k}_{j}\right)}{\mathrm{PV}_{y}\left\{\tilde{k}_{j}+k-\left(\frac{\tilde{k}_{j}}{\tilde{k}_{j}^{2}+m^{2} / 4+F}+\frac{k}{k^{2}+m^{2}+F}\right)\left[\left(\tilde{k}_{j}+k\right)^{2}+9 m^{2} / 4+F\right]\right\}}, \\
r_{j}=\frac{\left(k+2 \tilde{k}_{j}\right)}{\mathrm{PV}_{y}\left\{\tilde{k}_{j}+k-\left(\frac{\tilde{k}_{j}}{\tilde{k}_{j}^{2}+m^{2} / 4+F}+\frac{k}{k^{2}+m^{2}+F}\right)\left[\left(\tilde{k}_{j}+k\right)^{2}+m^{2} / 4+F\right]\right\}} \\
s_{j}=\frac{\left(k+2 \tilde{k}_{j}\right)}{\mathrm{PV}_{y}\left\{\tilde{k}_{j}-k-\left(\frac{\tilde{k}_{j}}{\tilde{k}_{j}^{2}+m^{2} / 4+F}-\frac{k}{k^{2}+m^{2}+F}\right)\left[\left(\tilde{k}_{j}-k\right)^{2}+9 m^{2} / 4+F\right]\right\}} \\
h_{j}=\frac{\left(k-2 \tilde{k}_{j}\right)}{\mathrm{PV}_{y}\left\{\tilde{k}_{j}-k-\left(\frac{\tilde{k}_{j}}{\tilde{k}_{j}^{2}+m^{2} / 4+F}-\frac{k}{k^{2}+m^{2}+F}\right)\left[\left(\tilde{k}_{j}-k\right)^{2}+m^{2} / 4+F\right]\right\}},
\end{gathered}
$$




$$
Q_{j}=k^{2}+m^{2}-\left(\tilde{k}_{j}^{2}+m^{2} / 4\right),(j=1,2) .
$$

\section{APPENDIX B}

\section{Coefficients of the Blocking Wave Packet Eq. (6h) in the Extended NMI Model}

The following equations define the coefficients of the nonlinearity and eddy forcing terms of the blocking wave packet Eq. (6h):

$$
\delta_{N}=\frac{k m \sum_{n=1}^{\infty} q_{N n} g_{n}^{2}\left[k^{2}+m^{2}-m^{2}(n+1 / 2)^{2}\right]}{k^{2}+m^{2}+F},
$$

$$
\begin{gathered}
q_{N n}=\frac{L_{y}\left\{1-\left(m^{2}+F-k^{2}\right)[F\right.}{L_{n}} \\
g_{n}=\frac{8}{m\left[4-(n+1 / 2)^{2}\right] L_{y}}, \\
G_{B}=\alpha \sqrt{\frac{L_{y}}{2} \frac{\left(\tilde{k}_{1}+\tilde{k}_{2}\right)^{2}\left(\tilde{k}_{2}-\tilde{k}_{1}\right) m}{4\left(k^{2}+m^{2}+F\right)}} \text { with } \quad \alpha=-1 .
\end{gathered}
$$

\section{REFERENCES}

Aikawa, T., M. Inatsu, N. Nakano, and T. Iwano, 2019: Modedecomposed equation diagnosis for atmospheric blocking development. J. Atmos. Sci., 76, 3151-3167, https://doi.org/ 10.1175/JAS-D-18-0362.1.

Berggren, R., B. Bolin, and C.-G. Rossby, 1949: An aerological study of zonal motion, its perturbations and break-down. Tellus, 1 (2), 14-37, https://doi.org/10.3402/tellusa.v1i2.8501.

Brunner, L., G. C. Hegerl, and A. K. Steiner, 2017: Connecting atmospheric blocking to European temperature extremes in spring. J. Climate, 30, 585-594, https://doi.org/10.1175/JCLID-16-0518.1.

Buehler, T., C. C. Raible and T. F. Stocker, 2011: The relationship of winter season North Atlantic blocking frequencies to extreme cold or dry spells in the ERA-40. Tellus, 63A , 174-187, https://doi.org/10.1111/j.1600-0870.2010.00492.x.

Cohen, J., and Coauthors, 2020: Divergent consensuses on Arctic amplification influence on midlatitude severe winter weather. Nat. Climate Change, 10, 20-29, https://doi.org/10.1038/ s41558-019-0662-y.

Colucci, S. J., 1985: Explosive cyclogenesis and large-scale circulation changes: Implications for atmospheric blocking. J. Atmos. Sci., 42, 2701-2717, https://doi.org/10.1175/15200469(1985)042<2701:ECALSC > 2.0.CO;2.

Della-Marta, P. M., J. Luterbacher, H. von Weissenfluh, E. Xoplaki, M. Brunet, and H. Wanner, 2007: Summer heat waves over western Europe 1880-2003, their relationship to large-scale forcings and predictability. Climate Dyn., 29, 251-275, https://doi.org/10.1007/s00382-007-0233-1.

Haines, K., and J. C. Marshall, 1987: Eddy-forced coherent structures as a prototype of atmospheric blocking. Quart. J. Roy. Meteor. Soc., 113, 681-704, https://doi.org/10.1002/qj.49711347613.
Holopainen, E., and C. Fortelius, 1987: High-frequency transient eddies and blocking. J. Atmos. Sci., 44, 1632-1645, https://doi.org/10.1175/1520-0469(1987)044<1632:HFTEAB> 2.0.CO;2.

Hoskins, B., and I. N. James, 2014: Fluid Dynamics of the Midlatitude Atmosphere. John Wiley and Sons, 432 pp.

Illari, L., and J. C. Marshall, 1983: On the interpretation of eddy fluxes during a blocking episode. J. Atmos. Sci., 40, 2232-2242, https://doi.org/10.1175/1520-0469(1983)040<2232: OTIOEF $>2.0 . \mathrm{CO} ; 2$.

Ji, L. R., and S. Tibaldi, 1983: Numerical simulations of a case of blocking: The effects of orography and land-sea contrast. Mon. Wea. Rev., 111, 2068-2086, https://doi.org/10.1175/15200493(1983)111<2068:NSOACO > 2.0.CO;2.

Kodera, K., H. Mukougawa, and A. Fujii, 2013: Influence of the vertical and zonal propagation of stratospheric planetary waves on tropospheric blockings. J. Geophys. Res. Atmos., 118, 8333-8345, https://doi.org/10.1002/JGRD.50650.

Li, M., and D. Luo, 2019: Winter Arctic warming and its linkage with midlatitude atmospheric circulation and associated cold extremes: The key role of meridional potential vorticity gradient. Sci. China Earth Sci., 62, 1329-1339, https://doi.org/ 10.1007/s11430-018-9350-9.

Luo, D., 2000: Planetary-scale baroclinic envelope Rossby solitons in a two-layer model and their interaction with synoptic-scale eddies. Dyn. Atmos. Oceans, 32, 27-74, https://doi.org/10.1016/ S0377-0265(99)00018-4.

_ 2005: A barotropic envelope Rossby soliton model for blockeddy interaction. Part I: Effect of topography. J. Atmos. Sci., 62, 5-21, https://doi.org/10.1175/1186.1.

_, J. Cha, L. Zhong, and A. Dai, 2014: A nonlinear multiscale interaction model for atmospheric blocking: The eddy-blocking matching mechanism. Quart. J. Roy. Meteor. Soc., 140, 17851808, https://doi.org/10.1002/qj.2337.

—, X. Chen, A. Dai, and I. Simmonds, 2018: Changes in atmospheric blocking circulations linked with winter Arctic warming: A new perspective. J. Climate, 31, 7661-7678, https:// doi.org/10.1175/JCLI-D-18-0040.1.

, W. Zhang, L. Zhong, and A. Dai, 2019: A nonlinear theory of atmospheric blocking: A potential vorticity gradient view. J. Atmos. Sci., 76, 2399-2427, https://doi.org/10.1175/JAS-D18-0324.1. 
Mullen, S. L., 1987: Transient eddy forcing of blocking flows. J. Atmos. Sci., 44, 3-22, https://doi.org/10.1175/1520-0469(1987) 044<0003:TEFOBF $>2.0 . \mathrm{CO} ; 2$.

Muslu, G. M., and H. A. Erbay, 2005: Higher-order split-step Fourier schemes for the generalized nonlinear Schrödinger equation. Math. Comput. Simul., 67, 581-595, https://doi.org/ 10.1016/j.matcom.2004.08.002.

Nakamura, H., and J. M. Wallace, 1993: Synoptic behavior of baroclinic eddies during the blocking onset. Mon. Wea. Rev., 121, 1892-1903, https://doi.org/10.1175/1520-0493(1993)121<1892: SBOBED $>2.0 . \mathrm{CO} ; 2$.

Nakamura, N., and C. Huang, 2017: Local wave activity and the onset of blocking along a potential vorticity front. J. Atmos. Sci., 74, 2341-2362, https://doi.org/10.1175/JASD-17-0029.1.

_ and —, 2018: Atmospheric blocking as a traffic jam in the jet stream. Science, 361, 42-47, https://doi.org/10.1126/ science.aat0721.

Newson, R. L., 1973: Response of general circulation model of the atmosphere to removal of the Arctic ice cap. Nature, 241, 3940, https://doi.org/10.1038/241039b0.

Paradise, A., C. B. Rocha, P. Barpanda, and N. Nakamura, 2019: Blocking statistics in a varying climate: Lessons from a "traffic jam" model with pseudostochastic forcing. J. Atmos. Sci., 76, 3013-3027, https://doi.org/10.1175/JAS-D-19-0095.1.
Rex, D. F., 1950: Blocking action in the middle troposphere and its effect upon regional climate. I: An aerological study of blocking action. Tellus, 2, 196-211, https://doi.org/10.1111/ j.2153-3490.1950.tb00331.x.

Schaller, N., J. Sillmann, J. Anstey, E. M. Fischer, C. M. Grams, and S. Russo, 2018: Influence of blocking on northern European and western Russian heatwaves in large climate model ensembles. Environ. Res. Lett., 13, 054015, https://doi.org/10.1088/1748-9326/ aaba55.

Shukla, J., and K. C. Mo, 1983: Seasonal and geographical variation of blocking. Mon. Wea. Rev., 111, 388-402, https://doi.org/ 10.1175/1520-0493(1983)111<0388:SAGVOB > 2.0.CO;2.

Shutts, G. J., 1983: The propagation of eddies in diffluent jetstreams: Eddy vorticity forcing of 'blocking' flow fields. Quart. J. Roy. Meteor. Soc., 109, 737-761, https://doi.org/10.1002/QJ.49710946204.

Weijenborg, C., H. deVries, and R. J. Haarsma, 2012: On the direction of Rossby wave breaking in blocking. Climate Dyn., 39, 2823-2831, https://doi.org/10.1007/s00382-012-1332-1.

Yeh, T. C., 1949: On energy dispersion in the atmosphere. J. Meteor., 6, 1-16, https://doi.org/10.1175/1520-0469(1949) $006<0001:$ OEDITA $>2.0$. CO; 2 .

Zhang, W., and D. Luo, 2020: A nonlinear theory of atmospheric blocking: An application to Greenland blocking changes linked to winter Arctic sea ice loss. J. Atmos. Sci., 77, 723-751, https://doi.org/10.1175/JAS-D-19-0198.1. 